Prepared for the U.S. Department of Energy under Contract DE-AC05-76RL01830

\title{
Report for Batch Leach Analyses on Sediments at 100-FR-3 Operable Unit, Boreholes C7790, C7791, and C7792
}

Michael Lindberg

March 2011

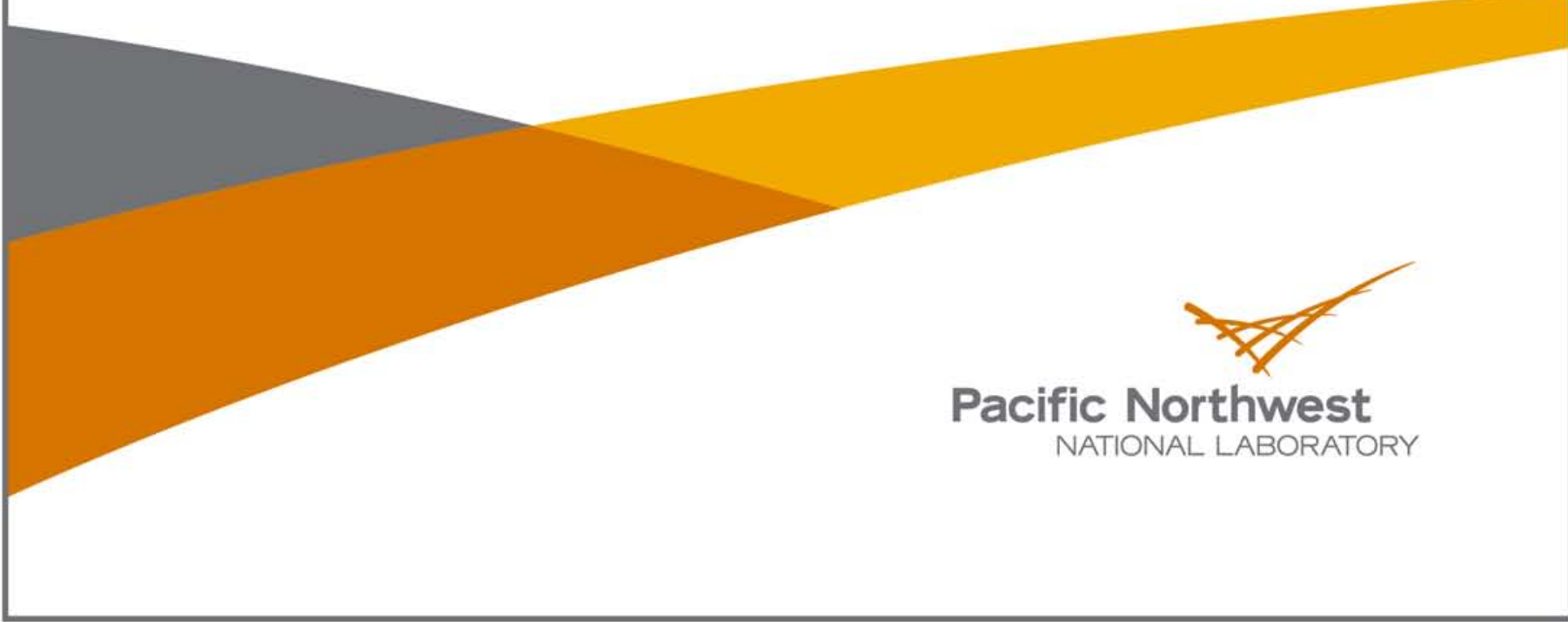




\title{
DISCLAIMER
}

This report was prepared as an account of work sponsored by an agency of the United States Government. Neither the United States Government nor any agency thereof, nor Battelle Memorial Institute, nor any of their employees, makes any warranty, express or implied, or assumes any legal liability or responsibility for the accuracy, completeness, or usefulness of any information, apparatus, product, or process disclosed, or represents that its use would not infringe privately owned rights. Reference herein to any specific commercial product, process, or service by trade name, trademark, manufacturer, or otherwise does not necessarily constitute or imply its endorsement, recommendation, or favoring by the United States Government or any agency thereof, or Battelle Memorial Institute. The views and opinions of authors expressed herein do not necessarily state or reflect those of the United States Government or any agency thereof.

\author{
PACIFIC NORTHWEST NATIONAL LABORATORY \\ operated by \\ BATTELLE \\ for the \\ UNITED STATES DEPARTMENT OF ENERGY \\ under Contract DE-AC05-76RL01830
}

Printed in the United States of America

Available to DOE and DOE contractors from the

Office of Scientific and Technical Information,

P.O. Box 62, Oak Ridge, TN 37831-0062;

ph: (865) 576-8401

fax: $(865) 576-5728$

email: reports@adonis.osti.gov

\footnotetext{
Available to the public from the National Technical Information Service, U.S. Department of Commerce, 5285 Port Royal Rd., Springfield, VA 22161 ph: (800) 553-6847 fax: $(703) 605-6900$

email: orders@ntis.fedworld.gov

online ordering: http://www.ntis.gov/ordering.htm
}

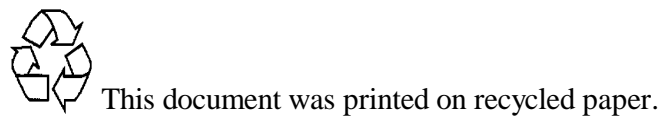




\section{Report for Batch Leach Analyses on Sediments at 100-FR-3 Operable Unit, Boreholes C7790, C7791, and C7792}

M Lindberg

March 2011

Prepared for the U.S. Department of Energy

under Contract DE-AC05-76RL01830

Pacific Northwest National Laboratory

Richland, Washington 99352 
$03 / 28 / 1114: 53$

To: Anna Radloff

From: Michael J. Lindberg

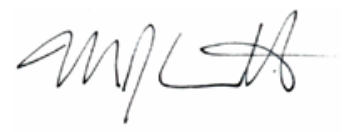

Environmental Sciences Laboratory

Energy and Environment Directorate, Pacific Northwest National Laboratory

Subject: Analytical Data Report for Sediment Samples Collected From 100-FR-3 Operable Unit, Sample Delivery Group ESL090018, SAF Number F10-234

This letter contains the following information for sample delivery group ESL090018

- Cover Sheet

- Narrative

- Analytical Results

- Quality Control

- Chain of Custodies 


\section{Introduction}

Between August 15, 2010 and December 2, 2010 sediment samples were received from the 100-FR-3 Operable Unit for geochemical studies.

\section{Analytical Results/Methodology}

The analyses for this project were performed at the 325 building located in the 300 Area of the Hanford Site. The analyses were performed according to Pacific Northwest National Laboratory (PNNL) approved procedures and/or nationally recognized test procedures. The data sets include the sample identification numbers, analytical results, estimated quantification limits (EQL), and quality control data.

\section{Quality Control}

The preparatory and analytical quality control requirements, calibration requirements, acceptance criteria, and failure actions are defined in the on-line QA plan "Conducting Analytical Work in Support of Regulatory Programs" (CAW). This QA plan implements the Hanford Analytical Services Quality Assurance Requirements Documents (HASQARD) for PNNL.

\section{Definitions}

Dup Duplicate

RPD Relative Percent Difference

NR No Recovery (percent recovery less than zero)

ND Non-Detectable

$\%$ REC Percent Recovery

\section{Sample Receipt}

Samples were received with a chain of custody (COC) and were analyzed according to the sample identification numbers supplied by the client. All Samples were refrigerated upon receipt until prepared for analysis.

All samples were received with custody seals intact unless noted in the Case Narrative.

\section{Holding Times}

Holding time is defined as the time from sample preparation to the time of analyses. The prescribed holding times were met for all analytes unless noted in the Case Narrative.

\section{Analytical Results}

All reported analytical results meet the requirements of the CAW or client specified SOW unless noted in the case narrative. 


\section{Case Narrative Report}

\section{Hold Time:}

Due to the requirements of the statement of work and sampling events in the field, the 28 day and the 48 hr requirements could not be met. All applicable hold times were started from the time of preparation and not the time of sampling.

\section{Preparation Blank (PB):}

No discrepancies noted.

\section{Duplicate (DUP):}

Duplicate RPD for Arsenic (55.5\%) was above the acceptance limit (35) in 0J11001-DUP1 for ICPMS-RCRA-AE Duplicate failure may be due to sample heterogeneity. All other duplicates and QC associated with the batch were in limits. There should be no impact to sample data as reported.

Duplicate RPD for Chromium (63.6\%) was above the acceptance limit (35) in 0J11001-DUP1 for ICPMS-RCRA-AE

Duplicate failure may be due to sample heterogeneity. All other duplicates and QC associated with the batch were in limits. There should be no impact to sample data as reported.

Duplicate RPD for Lead (50.3\%) was above the acceptance limit (35) in 0J11001-DUP2 for ICPMS-RCRA-AE

Duplicate failure may be due to sample heterogeneity. All other duplicates and QC associated with the batch were in limits. There should be no impact to sample data as reported.

Duplicate RPD for Barium (50.1\%) was above the acceptance limit (35) in 0J14004-DUP1 for ICP-OES Vadose-AE

Duplicate failure may be due to sample heterogeneity. All other duplicates and QC associated with the batch were in limits. There should be no impact to sample data as reported.

\section{Laboratory Control Samples (LCS):}

No discrepancies noted.

\section{Post Spike (PS):}

Post-Spike Recovery for Chromium, Hexavalent (153\%) was outside acceptance limits (75-125) in 0J15001-PS1 for Hexavalent Chromium/WE 1:5

The high recovery for the post spike is due to a preparation error. Sample results associated with this batch are below the EQL and all other batch QC was in limits. There should be no impact to the data as reported.

\section{Matrix Spike (MS):}

No discrepancies noted.

\section{Other QC Criteria:}

No discrepancies noted. 
I certify that this data package is in compliance with the SOW, both technically and for completeness, for other than the conditions detailed above. Release of the data contained in this hard copy data package has been authorized by the Laboratory Analytical Manager as verified by this signature.

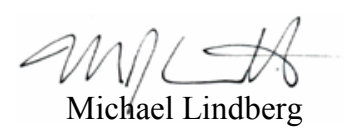

\section{DISCLAIMER}

This report was prepared as an account of work sponsored by an agency of the United States Government. Neither the United States Government nor any agency thereof, nor Battelle Memorial Institute, nor any of their employees, makes any warranty, express or implied, or assumes any legal liability or responsibility for the accuracy, completeness, or usefulness of any information, apparatus, product, or process disclosed, or represents that its use would not infringe privately owned rights. Reference herein to any specific commercial product, process, or service by trade name, trademark, manufacturer, or otherwise does not necessarily constitute or imply its endorsement, recommendation, or favoring by the United States Government or any agency thereof, or Battelle Memorial Institute. The views and opinions of authors expressed herein do not necessarily state or reflect those of the United States Government or any agency thereof. 
The following analyses were performed on the following samples included in this report:

Metals 1:1 DI Water Extract by ICPMS

Metals 1:2.5 DI Water Extract by ICPMS

Metals 1:5 DI Water Extract by ICPMS

Metals Acid Extract by ICPMS

Hexavalent Chromium by Colorimetric Determination

Metals 1:1 Water Extract by ICPOES

Metals 1:2.5 Water Extract by ICPOES

Metals 1:5 Water Extract by ICPOES

Metals Acid Extract by ICPOES

Moisture Content

Stronium 90 Acid Extract by LSC

Strontium 90 1:2.5 DI Water Extract by LSC

Strontium 90 1:5 DI Water Extract by LSC

Strontium 90 Water Extract by LSC

\section{SAMPLES ANALYZED IN THIS REPORT}

$\begin{array}{lll}\text { HEIS No. } & \begin{array}{l}\text { Laboratory ID } \\ 1009008-01\end{array} & \text { Matrix } \\ \text { B274K7 } & \text { SOIL } \\ \text { B273B8 } & 1009008-02 & \text { SOIL } \\ \text { B27PY0 } & 1009008-03 & \text { SOIL } \\ \text { B274K6 } & 1009008-04 & \text { SOIL } \\ \text { B26TC1 } & 1009008-05 & \text { SOIL } \\ \text { B26TC2 } & 1009008-06 & \text { SOIL } \\ \text { B273B2 } & 1009008-07 & \text { SOIL } \\ \text { B273B3 } & 1009008-08 & \text { SOIL } \\ \text { B273B4 } & 1009008-09 & \text { SOIL } \\ \text { B273B6 } & 1009008-10 & \text { SOIL } \\ \text { B273B5 } & 1009008-11 & \text { SOIL }\end{array}$

\begin{tabular}{|c|c|}
\hline $\begin{array}{l}\text { Date Collected } \\
9 / 1 / 1010: 26\end{array}$ & $\begin{array}{l}\text { Date Received } \\
9 / 1 / 1015: 00\end{array}$ \\
\hline $8 / 30 / 10 \quad 12: 16$ & $9 / 1 / 10 \quad 15: 00$ \\
\hline $8 / 31 / 10 \quad 08: 57$ & $9 / 1 / 10 \quad 15: 00$ \\
\hline $8 / 26 / 10 \quad 14: 00$ & $8 / 30 / 10 \quad 14: 00$ \\
\hline $8 / 13 / 10 \quad 12: 25$ & $8 / 15 / 10 \quad 14: 45$ \\
\hline $8 / 16 / 10 \quad 10: 00$ & $8 / 15 / 10 \quad 14: 45$ \\
\hline $11 / 11 / 10 \quad 08: 50$ & $11 / 16 / 10 \quad 08: 40$ \\
\hline $11 / 15 / 10 \quad 08: 30$ & $11 / 16 / 10 \quad 08: 40$ \\
\hline $11 / 15 / 10 \quad 08: 30$ & $11 / 16 / 10 \quad 08: 40$ \\
\hline $11 / 29 / 10 \quad 09: 50$ & $12 / 2 / 10 \quad 09: 05$ \\
\hline $11 / 22 / 10 \quad 11: 10$ & $12 / 2 / 10 \quad 09: 05$ \\
\hline
\end{tabular}




\section{Wet Chemistry}

Moisture Content (\% by Weight) by AGG-WC-001

\begin{tabular}{|c|c|c|c|c|c|}
\hline Lab ID & HEIS No. & Results & EQL & Analyzed & Batch \\
\hline $1009008-01$ & B274K7 & $3.51 \mathrm{E} 1$ & $\mathrm{~N} / \mathrm{A}$ & $10 / 05 / 10$ & 0I29004 \\
\hline $1009008-02$ & B273B8 & $2.19 \mathrm{E} 1$ & N/A & $10 / 05 / 10$ & 0I29004 \\
\hline $1009008-03$ & B27PY0 & $1.39 \mathrm{E} 1$ & N/A & $10 / 05 / 10$ & 0I29004 \\
\hline $1009008-04$ & B274K6 & $2.36 \mathrm{E} 1$ & N/A & $10 / 05 / 10$ & 0I29004 \\
\hline $1009008-05$ & B26TC1 & $1.12 \mathrm{E} 1$ & N/A & $10 / 05 / 10$ & 0I29004 \\
\hline 1009008-06 & B26TC2 & $2.49 \mathrm{E} 1$ & N/A & $10 / 05 / 10$ & 0I29004 \\
\hline 1009008-07 & B273B2 & 4.73E0 & $\mathrm{N} / \mathrm{A}$ & $12 / 30 / 10$ & 0L27001 \\
\hline $1009008-08$ & B273B3 & $1.97 \mathrm{E} 1$ & N/A & $12 / 30 / 10$ & 0L27001 \\
\hline 1009008-09 & B273B4 & $1.15 \mathrm{E} 1$ & N/A & $12 / 30 / 10$ & 0L27001 \\
\hline $1009008-10$ & B273B6 & $3.81 \mathrm{E} 1$ & N/A & $12 / 30 / 10$ & 0L27001 \\
\hline $1009008-11$ & B273B5 & $3.08 \mathrm{E} 1$ & N/A & $12 / 30 / 10$ & 0L27001 \\
\hline
\end{tabular}


Hexavalent Chromium/Soil

Chromium, Hexavalent (ug/g dry) by Colorimetric Determination

\begin{tabular}{llcccc|} 
Lab ID & HEIS No. & Results & EQL & Analyzed & Batch \\
\hline $1009008-01$ & B274K7 & $<6.72 \mathrm{E}-1$ & $6.72 \mathrm{E}-1$ & $10 / 14 / 10$ & $0 \mathrm{~J} 14008$ \\
$1009008-02$ & B273B8 & $<5.92 \mathrm{E}-1$ & $5.92 \mathrm{E}-1$ & $10 / 14 / 10$ & $0 \mathrm{~J} 14008$ \\
$1009008-03$ & B27PY0 & $<5.27 \mathrm{E}-1$ & $5.27 \mathrm{E}-1$ & $10 / 14 / 10$ & $0 \mathrm{~J} 14008$ \\
$1009008-04$ & B274K6 & $<6.11 \mathrm{E}-1$ & $6.11 \mathrm{E}-1$ & $10 / 14 / 10$ & $0 \mathrm{~J} 14008$ \\
$1009008-05$ & B26TC1 & $<5.25 \mathrm{E}-1$ & $5.25 \mathrm{E}-1$ & $10 / 14 / 10$ & $0 \mathrm{~J} 14008$ \\
$1009008-06$ & B26TC2 & $<5.76 \mathrm{E}-1$ & $5.76 \mathrm{E}-1$ & $10 / 14 / 10$ & $0 \mathrm{~J} 14008$ \\
$1009008-07$ & B273B2 & $<5.22 \mathrm{E}-1$ & $5.22 \mathrm{E}-1$ & $1 / 13 / 11$ & $1 \mathrm{~A} 14001$ \\
$1009008-08$ & B273B3 & $<5.98 \mathrm{E}-1$ & $5.98 \mathrm{E}-1$ & $1 / 13 / 11$ & $1 \mathrm{~A} 14001$ \\
$1009008-09$ & B273B4 & $<5.55 \mathrm{E}-1$ & $5.55 \mathrm{E}-1$ & $1 / 13 / 11$ & $1 \mathrm{~A} 14001$ \\
$1009008-10$ & B273B6 & $<6.93 \mathrm{E}-1$ & $6.93 \mathrm{E}-1$ & $1 / 13 / 11$ & $1 \mathrm{~A} 14001$ \\
$1009008-11$ & B273B5 & $<6.54 \mathrm{E}-1$ & $6.54 \mathrm{E}-1$ & $1 \mathrm{~A} 14001$ \\
\hline
\end{tabular}


Hexavalent Chromium/1:1 Water Extract

Chromium, Hexavalent (ug/g dry) by Colorimetric Determination

\begin{tabular}{llcccc} 
Lab ID & HEIS No. & Results & EQL & Analyzed & Batch \\
\hline $1009008-01$ & B274K7 & $<2.94 \mathrm{E}-2$ & $2.94 \mathrm{E}-2$ & $10 / 11 / 10$ & $0 \mathrm{~J} 11006$ \\
$1009008-02$ & B273B8 & $<3.90 \mathrm{E}-2$ & $3.90 \mathrm{E}-2$ & $10 / 11 / 10$ & $0 \mathrm{~J} 11006$ \\
$1009008-03$ & B27PY0 & $<4.37 \mathrm{E}-2$ & $4.37 \mathrm{E}-2$ & $10 / 11 / 10$ & $0 \mathrm{~J} 11006$ \\
$1009008-04$ & B274K6 & $<3.83 \mathrm{E}-2$ & $3.83 \mathrm{E}-2$ & $10 / 11 / 10$ & $0 \mathrm{~J} 11006$ \\
$1009008-05$ & B26TC1 & $<4.45 \mathrm{E}-2$ & $4.45 \mathrm{E}-2$ & $10 / 11 / 10$ & $0 \mathrm{~J} 11006$ \\
$1009008-06$ & B26TC2 & $<3.97 \mathrm{E}-2$ & $3.97 \mathrm{E}-2$ & $10 / 11 / 10$ & $0 \mathrm{~J} 11006$ \\
$1009008-07$ & B273B2 & $<4.76 \mathrm{E}-2$ & $4.76 \mathrm{E}-2$ & $1 / 07 / 11$ & $1 \mathrm{~A} 06002$ \\
$1009008-08$ & B273B3 & $<4.02 \mathrm{E}-2$ & $4.02 \mathrm{E}-2$ & $1 / 07 / 11$ & $1 \mathrm{~A} 06002$ \\
$1009008-09$ & B273B4 & $<4.43 \mathrm{E}-2$ & $4.43 \mathrm{E}-2$ & $1 / 07 / 11$ & $1 \mathrm{~A} 06002$ \\
$1009008-10$ & B273B6 & $<3.06 \mathrm{E}-2$ & $3.06 \mathrm{E}-2$ & $1 / 07 / 11$ & $1 \mathrm{~A} 06002$ \\
$1009008-11$ & B273B5 & $<3.62 \mathrm{E}-2$ & $3.62 \mathrm{E}-2$ & $1 / 07 / 11$ & $1 \mathrm{~A} 06002$ \\
\hline
\end{tabular}


Hexavalent Chromium/1:2.5 Water Extract

Chromium, Hexavalent (ug/g dry) by Colorimetric Determination

\begin{tabular}{llcccc|} 
Lab ID & HEIS No. & Results & EQL & Analyzed & Batch \\
\hline $1009008-01$ & B274K7 & $<1.02 \mathrm{E}-1$ & $1.02 \mathrm{E}-1$ & $12 / 02 / 10$ & $0 \mathrm{~J} 12002$ \\
$1009008-02$ & B273B8 & $<1.14 \mathrm{E}-1$ & $1.14 \mathrm{E}-1$ & $12 / 02 / 10$ & $0 \mathrm{~J} 12002$ \\
$1009008-03$ & B27PY0 & $<1.18 \mathrm{E}-1$ & $1.18 \mathrm{E}-1$ & $12 / 02 / 10$ & $0 \mathrm{~J} 12002$ \\
$1009008-04$ & B274K6 & $<1.22 \mathrm{E}-1$ & $1.22 \mathrm{E}-1$ & $12 / 02 / 10$ & $0 \mathrm{~J} 12002$ \\
$1009008-05$ & B26TC1 & $<1.19 \mathrm{E}-1$ & $1.19 \mathrm{E}-1$ & $12 / 02 / 10$ & $0 \mathrm{~J} 12002$ \\
$1009008-06$ & B26TC2 & $<1.12 \mathrm{E}-1$ & $1.12 \mathrm{E}-1$ & $12 / 02 / 10$ & $0 \mathrm{~J} 12002$ \\
$1009008-07$ & B273B2 & $<1.21 \mathrm{E}-1$ & $1.21 \mathrm{E}-1$ & $1 / 07 / 11$ & $1 \mathrm{~A} 07001$ \\
$1009008-08$ & B273B3 & $<1.14 \mathrm{E}-1$ & $1.14 \mathrm{E}-1$ & $1 / 07 / 11$ & $1 \mathrm{~A} 07001$ \\
$1009008-09$ & B273B4 & $<1.19 \mathrm{E}-1$ & $1.19 \mathrm{E}-1$ & $1 / 07 / 11$ & $1 \mathrm{~A} 07001$ \\
$1009008-10$ & B273B6 & $<1.06 \mathrm{E}-1$ & $1.06 \mathrm{E}-1$ & $1 / 07 / 11$ & $1 \mathrm{~A} 07001$ \\
$1009008-11$ & B273B5 & $<1.10 \mathrm{E}-1$ & $1.10 \mathrm{E}-1$ & $1 \mathrm{~A} 07001$ \\
\hline
\end{tabular}


Hexavalent Chromium/1:5 Water Extract

Chromium, Hexavalent (ug/g dry) by Colorimetric Determination

\begin{tabular}{llcccc|} 
Lab ID & HEIS No. & Results & EQL & Analyzed & Batch \\
\hline $1009008-01$ & B274K7 & $<2.22 \mathrm{E}-1$ & $2.22 \mathrm{E}-1$ & $12 / 02 / 10$ & $0 \mathrm{~J} 15001$ \\
$1009008-02$ & B273B8 & $<2.36 \mathrm{E}-1$ & $2.36 \mathrm{E}-1$ & $12 / 02 / 10$ & $0 \mathrm{~J} 15001$ \\
$1009008-03$ & B27PY0 & $<2.23 \mathrm{E}-1$ & $2.23 \mathrm{E}-1$ & $12 / 02 / 10$ & $0 \mathrm{~J} 15001$ \\
$1009008-04$ & B274K6 & $<2.38 \mathrm{E}-1$ & $2.38 \mathrm{E}-1$ & $12 / 02 / 10$ & $0 \mathrm{~J} 15001$ \\
$1009008-05$ & B26TC1 & $<2.40 \mathrm{E}-1$ & $2.40 \mathrm{E}-1$ & $12 / 02 / 10$ & $0 \mathrm{~J} 15001$ \\
$1009008-06$ & B26TC2 & $<2.37 \mathrm{E}-1$ & $2.37 \mathrm{E}-1$ & $12 / 02 / 10$ & $0 \mathrm{~J} 15001$ \\
$1009008-07$ & B273B2 & $<2.48 \mathrm{E}-1$ & $2.48 \mathrm{E}-1$ & $1 / 07 / 11$ & $1 \mathrm{~A} 07002$ \\
$1009008-08$ & B273B3 & $<2.37 \mathrm{E}-1$ & $2.37 \mathrm{E}-1$ & $1 / 07 / 11$ & $1 \mathrm{~A} 07002$ \\
$1009008-09$ & B273B4 & $<2.45 \mathrm{E}-1$ & $2.45 \mathrm{E}-1$ & $1 / 07 / 11$ & $1 \mathrm{~A} 07002$ \\
$1009008-10$ & B273B6 & $<2.30 \mathrm{E}-1$ & $2.30 \mathrm{E}-1$ & $1 / 07 / 11$ & $1 \mathrm{~A} 07002$ \\
$1009008-11$ & B273B5 & $<2.29 \mathrm{E}-1$ & $2.29 \mathrm{E}-1$ & $1 \mathrm{~A} 07002$ \\
\hline
\end{tabular}


Total Metals by PNNL-AGG-ICP-AES/1:1 Water Extract

\begin{tabular}{|c|c|c|c|c|c|c|}
\hline CAS \# & Analyte & Results & Units & Analyzed & Batch & Method \\
\hline HEIS No. & B274K7 & \multicolumn{2}{|c|}{ Lab ID: $\quad$ 1009008-01 } & & & \\
\hline $7440-39-3$ & Barium & $<1.16 \mathrm{E}-1$ & $\mathrm{ug} / \mathrm{g}$ dry $\quad 1.16 \mathrm{E}-1$ & $10 / 14 / 10$ & $0 \mathrm{~J} 14005$ & PNNL-AGG-ICP-AES \\
\hline HEIS No. & B273B8 & \multicolumn{2}{|c|}{ Lab ID: $\quad$ 1009008-02 } & & & \\
\hline $7440-39-3$ & Barium & $<1.24 \mathrm{E}-1$ & $\mathrm{ug} / \mathrm{g}$ dry $\quad 1.24 \mathrm{E}-1$ & $10 / 14 / 10$ & $0 \mathrm{~J} 14005$ & PNNL-AGG-ICP-AES \\
\hline HEIS No. & B27PY0 & \multicolumn{2}{|c|}{ Lab ID: $\quad$ 1009008-03 } & & & \\
\hline $7440-39-3$ & Barium & $<1.25 \mathrm{E}-1$ & $\mathrm{ug} / \mathrm{g}$ dry $\quad 1.25 \mathrm{E}-1$ & $10 / 14 / 10$ & $0 \mathrm{~J} 14005$ & PNNL-AGG-ICP-AES \\
\hline HEIS No. & B274K6 & \multicolumn{2}{|c|}{ Lab ID: $\quad$ 1009008-04 } & & & \\
\hline $7440-39-3$ & Barium & $<1.24 \mathrm{E}-1$ & $\mathrm{ug} / \mathrm{g}$ dry $\quad 1.24 \mathrm{E}-1$ & $10 / 14 / 10$ & $0 \mathrm{~J} 14005$ & PNNL-AGG-ICP-AES \\
\hline HEIS No. & B26TC1 & \multicolumn{2}{|c|}{ Lab ID: $\quad$ 1009008-05 } & & & \\
\hline $7440-39-3$ & Barium & $<1.24 \mathrm{E}-1$ & $\mathrm{ug} / \mathrm{g}$ dry $\quad 1.24 \mathrm{E}-1$ & $10 / 14 / 10$ & $0 \mathrm{~J} 14005$ & PNNL-AGG-ICP-AES \\
\hline HEIS No. & B26TC2 & \multicolumn{2}{|c|}{ Lab ID: $\quad$ 1009008-06 } & & & \\
\hline $7440-39-3$ & Barium & $<1.29 \mathrm{E}-1$ & $\mathrm{ug} / \mathrm{g}$ dry $\quad 1.29 \mathrm{E}-1$ & $10 / 14 / 10$ & $0 \mathrm{~J} 14005$ & PNNL-AGG-ICP-AES \\
\hline HEIS No. & B273B2 & \multicolumn{2}{|c|}{ Lab ID: $\quad$ 1009008-07 } & & & \\
\hline $7440-39-3$ & Barium & $<8.25 \mathrm{E}-2$ & $\mathrm{ug} / \mathrm{g}$ dry $\quad 8.25 \mathrm{E}-2$ & $2 / 01 / 11$ & $1 \mathrm{~A} 31003$ & PNNL-AGG-ICP-AES \\
\hline HEIS No. & B273B3 & \multicolumn{2}{|c|}{ Lab ID: $\quad$ 1009008-08 } & & & \\
\hline $7440-39-3$ & Barium & $<8.26 \mathrm{E}-2$ & $\mathrm{ug} / \mathrm{g}$ dry $\quad 8.26 \mathrm{E}-2$ & $2 / 01 / 11$ & $1 \mathrm{~A} 31003$ & PNNL-AGG-ICP-AES \\
\hline HEIS No. & B273B4 & \multicolumn{2}{|c|}{ Lab ID: $\quad$ 1009008-09 } & & & \\
\hline $7440-39-3$ & Barium & $<8.26 \mathrm{E}-2$ & $\mathrm{ug} / \mathrm{g}$ dry $\quad 8.26 \mathrm{E}-2$ & $2 / 01 / 11$ & $1 \mathrm{~A} 31003$ & PNNL-AGG-ICP-AES \\
\hline HEIS No. & B273B6 & \multicolumn{2}{|c|}{ Lab ID: $\quad$ 1009008-10 } & & & \\
\hline $7440-39-3$ & Barium & $<8.20 \mathrm{E}-2$ & $\mathrm{ug} / \mathrm{g}$ dry $\quad 8.20 \mathrm{E}-2$ & $2 / 01 / 11$ & $1 \mathrm{~A} 31003$ & PNNL-AGG-ICP-AES \\
\hline HEIS No. & B273B5 & \multicolumn{2}{|c|}{ Lab ID: $\quad 1009008-11$} & & & \\
\hline $7440-39-3$ & Barium & $<8.52 \mathrm{E}-2$ & $\mathrm{ug} / \mathrm{g}$ dry $\quad 8.52 \mathrm{E}-2$ & $2 / 01 / 11$ & $1 \mathrm{~A} 31003$ & PNNL-AGG-ICP-AES \\
\hline
\end{tabular}


Total Metals by PNNL-AGG-ICP-AES/1:2.5 Water Extract

\begin{tabular}{|c|c|c|c|c|c|c|}
\hline CAS \# & Analyte & Results & Units & Analyzed & Batch & Method \\
\hline HEIS No. & B274K7 & \multicolumn{2}{|c|}{ Lab ID: $\quad$ 1009008-01 } & & & \\
\hline $7440-39-3$ & Barium & $<2.95 \mathrm{E}-1$ & ug/g dry $\quad 2.95 \mathrm{E}-1$ & $12 / 02 / 10$ & $0 \mathrm{~J} 14006$ & PNNL-AGG-ICP-AES \\
\hline HEIS No. & B273B8 & \multicolumn{2}{|c|}{ Lab ID: $\quad$ 1009008-02 } & & & \\
\hline $7440-39-3$ & Barium & $<3.08 \mathrm{E}-1$ & ug/g dry $3.08 \mathrm{E}-1$ & $12 / 02 / 10$ & 0J14006 & PNNL-AGG-ICP-AES \\
\hline HEIS No. & B27PY0 & \multicolumn{2}{|c|}{ Lab ID: $\quad$ 1009008-03 } & & & \\
\hline $7440-39-3$ & Barium & $<3.10 \mathrm{E}-1$ & ug/g dry $3.10 \mathrm{E}-1$ & $12 / 02 / 10$ & $0 \mathrm{~J} 14006$ & PNNL-AGG-ICP-AES \\
\hline HEIS No. & B274K6 & \multicolumn{2}{|c|}{ Lab ID: $\quad$ 1009008-04 } & & & \\
\hline $7440-39-3$ & Barium & $<3.30 \mathrm{E}-1$ & ug/g dry $\quad 3.30 \mathrm{E}-1$ & $12 / 02 / 10$ & 0J14006 & PNNL-AGG-ICP-AES \\
\hline HEIS No. & B26TC1 & \multicolumn{2}{|c|}{ Lab ID: $\quad$ 1009008-05 } & & & \\
\hline $7440-39-3$ & Barium & $<3.08 \mathrm{E}-1$ & ug/g dry $\quad 3.08 \mathrm{E}-1$ & $12 / 02 / 10$ & $0 \mathrm{~J} 14006$ & PNNL-AGG-ICP-AES \\
\hline HEIS No. & B26TC2 & \multicolumn{2}{|c|}{ Lab ID: $\quad$ 1009008-06 } & & & \\
\hline $7440-39-3$ & Barium & $<3.07 \mathrm{E}-1$ & ug/g dry $\quad 3.07 \mathrm{E}-1$ & $12 / 02 / 10$ & $0 \mathrm{~J} 14006$ & PNNL-AGG-ICP-AES \\
\hline HEIS No. & B273B2 & \multicolumn{2}{|c|}{ Lab ID: $\quad$ 1009008-07 } & & & \\
\hline $7440-39-3$ & Barium & $<2.04 \mathrm{E}-1$ & ug/g dry $\quad 2.04 \mathrm{E}-1$ & $2 / 01 / 11$ & $1 \mathrm{~A} 31004$ & PNNL-AGG-ICP-AES \\
\hline HEIS No. & B273B3 & \multicolumn{2}{|c|}{ Lab ID: $\quad$ 1009008-08 } & & & \\
\hline $7440-39-3$ & Barium & $<2.05 \mathrm{E}-1$ & ug/g dry $\quad 2.05 E-1$ & $2 / 01 / 11$ & $1 \mathrm{~A} 31004$ & PNNL-AGG-ICP-AES \\
\hline HEIS No. & B273B4 & \multicolumn{2}{|c|}{ Lab ID: $\quad$ 1009008-09 } & & & \\
\hline $7440-39-3$ & Barium & $<2.05 \mathrm{E}-1$ & ug/g dry $\quad 2.05 E-1$ & $2 / 01 / 11$ & $1 \mathrm{~A} 31004$ & PNNL-AGG-ICP-AES \\
\hline HEIS No. & B273B6 & \multicolumn{2}{|c|}{ Lab ID: $\quad$ 1009008-10 } & & & \\
\hline $7440-39-3$ & Barium & $<2.06 \mathrm{E}-1$ & $\mathrm{ug} / \mathrm{g}$ dry $\quad 2.06 \mathrm{E}-1$ & $2 / 01 / 11$ & $1 \mathrm{~A} 31004$ & PNNL-AGG-ICP-AES \\
\hline HEIS No. & B273B5 & \multicolumn{2}{|c|}{ Lab ID: $\quad 1009008-11$} & & & \\
\hline $7440-39-3$ & Barium & $<2.06 \mathrm{E}-1$ & ug/g dry $\quad 2.06 \mathrm{E}-1$ & $2 / 01 / 11$ & 1A31004 & PNNL-AGG-ICP-AES \\
\hline
\end{tabular}


Total Metals by PNNL-AGG-ICP-AES/1:5 Water Extract

\begin{tabular}{|c|c|c|c|c|c|c|}
\hline CAS \# & Analyte & Results & Units & Analyzed & Batch & Method \\
\hline HEIS No. & B274K7 & \multicolumn{2}{|c|}{ Lab ID: $\quad$ 1009008-01 } & & & \\
\hline $7440-39-3$ & Barium & $<5.94 \mathrm{E}-1$ & ug/g dry $\quad 5.94 \mathrm{E}-1$ & $12 / 02 / 10$ & 0J14007 & PNNL-AGG-ICP-AES \\
\hline HEIS No. & B273B8 & \multicolumn{2}{|c|}{ Lab ID: $\quad$ 1009008-02 } & & & \\
\hline $7440-39-3$ & Barium & $<6.13 \mathrm{E}-1$ & ug/g dry $\quad 6.13 \mathrm{E}-1$ & $12 / 02 / 10$ & 0J14007 & PNNL-AGG-ICP-AES \\
\hline HEIS No. & B27PY0 & \multicolumn{2}{|c|}{ Lab ID: $\quad$ 1009008-03 } & & & \\
\hline $7440-39-3$ & Barium & $<5.69 \mathrm{E}-1$ & ug/g dry $5.69 \mathrm{E}-1$ & $12 / 02 / 10$ & 0J14007 & PNNL-AGG-ICP-AES \\
\hline HEIS No. & B274K6 & \multicolumn{2}{|c|}{ Lab ID: $\quad$ 1009008-04 } & & & \\
\hline $7440-39-3$ & Barium & $<6.18 \mathrm{E}-1$ & ug/g dry $\quad 6.18 \mathrm{E}-1$ & $12 / 02 / 10$ & 0J14007 & PNNL-AGG-ICP-AES \\
\hline HEIS No. & B26TC1 & \multicolumn{2}{|c|}{ Lab ID: $\quad$ 1009008-05 } & & & \\
\hline $7440-39-3$ & Barium & $<6.09 \mathrm{E}-1$ & ug/g dry $\quad 6.09 E-1$ & $12 / 02 / 10$ & $0 \mathrm{~J} 14007$ & PNNL-AGG-ICP-AES \\
\hline HEIS No. & B26TC2 & \multicolumn{2}{|c|}{ Lab ID: $\quad$ 1009008-06 } & & & \\
\hline $7440-39-3$ & Barium & $<6.17 \mathrm{E}-1$ & ug/g dry $\quad 6.17 \mathrm{E}-1$ & $12 / 02 / 10$ & $0 \mathrm{~J} 14007$ & PNNL-AGG-ICP-AES \\
\hline HEIS No. & B273B2 & \multicolumn{2}{|c|}{ Lab ID: $\quad$ 1009008-07 } & & & \\
\hline $7440-39-3$ & Barium & $<4.12 \mathrm{E}-1$ & $\mathrm{ug} / \mathrm{g}$ dry $\quad 4.12 \mathrm{E}-1$ & $2 / 01 / 11$ & $1 \mathrm{~A} 31005$ & PNNL-AGG-ICP-AES \\
\hline HEIS No. & B273B3 & \multicolumn{2}{|c|}{ Lab ID: $\quad$ 1009008-08 } & & & \\
\hline $7440-39-3$ & Barium & $<4.07 \mathrm{E}-1$ & ug/g dry $\quad 4.07 \mathrm{E}-1$ & $2 / 01 / 11$ & $1 \mathrm{~A} 31005$ & PNNL-AGG-ICP-AES \\
\hline HEIS No. & B273B4 & \multicolumn{2}{|c|}{ Lab ID: $\quad$ 1009008-09 } & & & \\
\hline $7440-39-3$ & Barium & $<4.14 \mathrm{E}-1$ & ug/g dry $\quad 4.14 \mathrm{E}-1$ & $2 / 01 / 11$ & $1 \mathrm{~A} 31005$ & PNNL-AGG-ICP-AES \\
\hline HEIS No. & B273B6 & \multicolumn{2}{|c|}{ Lab ID: $\quad$ 1009008-10 } & & & \\
\hline $7440-39-3$ & Barium & $<4.11 \mathrm{E}-1$ & $\mathrm{ug} / \mathrm{g}$ dry $\quad 4.11 \mathrm{E}-1$ & $2 / 01 / 11$ & $1 \mathrm{~A} 31005$ & PNNL-AGG-ICP-AES \\
\hline HEIS No. & B273B5 & \multicolumn{2}{|c|}{ Lab ID: $\quad 1009008-11$} & & & \\
\hline $7440-39-3$ & Barium & $<4.03 \mathrm{E}-1$ & ug/g dry $\quad 4.03 \mathrm{E}-1$ & $2 / 01 / 11$ & $1 \mathrm{~A} 31005$ & PNNL-AGG-ICP-AES \\
\hline
\end{tabular}


Total Metals by PNNL-AGG-ICP-AES/Acid Extract

\begin{tabular}{|c|c|c|c|c|c|c|c|}
\hline CAS \# & Analyte & Results & Units & EQL & Analyzed & Batch & Method \\
\hline HEIS No. & B274K7 & \multicolumn{3}{|c|}{ Lab ID: $\quad$ 1009008-01 } & & & \\
\hline $7440-39-3$ & Barium & $3.12 \mathrm{E} 1$ & ug/g dry & $1.91 \mathrm{E} 0$ & $10 / 15 / 10$ & 0J14004 & PNNL-AGG-ICP-AES \\
\hline HEIS No. & B273B8 & \multicolumn{3}{|c|}{ Lab ID: $\quad$ 1009008-02 } & & & \\
\hline $7440-39-3$ & Barium & $2.54 \mathrm{E} 1$ & ug/g dry & $1.71 \mathrm{E} 0$ & $10 / 15 / 10$ & 0J14004 & PNNL-AGG-ICP-AES \\
\hline HEIS No. & B27PY0 & \multicolumn{3}{|c|}{ Lab ID: $\quad$ 1009008-03 } & & & \\
\hline $7440-39-3$ & Barium & $3.33 \mathrm{E} 1$ & ug/g dry & $1.59 \mathrm{E} 0$ & $10 / 15 / 10$ & 0J14004 & PNNL-AGG-ICP-AES \\
\hline HEIS No. & B274K6 & \multicolumn{3}{|c|}{ Lab ID: $\quad$ 1009008-04 } & & & \\
\hline $7440-39-3$ & Barium & $2.18 \mathrm{E} 1$ & ug/g dry & $1.72 \mathrm{E} 0$ & $10 / 15 / 10$ & 0J14004 & PNNL-AGG-ICP-AES \\
\hline HEIS No. & B26TC1 & \multicolumn{3}{|c|}{ Lab ID: $\quad$ 1009008-05 } & & & \\
\hline $7440-39-3$ & Barium & $2.96 \mathrm{E} 1$ & ug/g dry & $1.55 \mathrm{E} 0$ & $10 / 15 / 10$ & 0J14004 & PNNL-AGG-ICP-AES \\
\hline HEIS No. & B26TC2 & \multicolumn{3}{|c|}{ Lab ID: $\quad$ 1009008-06 } & & & \\
\hline $7440-39-3$ & Barium & $1.12 \mathrm{E} 2$ & ug/g dry & $1.72 \mathrm{E} 0$ & $10 / 15 / 10$ & 0J14004 & PNNL-AGG-ICP-AES \\
\hline HEIS No. & B273B2 & \multicolumn{3}{|c|}{ Lab ID: $\quad$ 1009008-07 } & & & \\
\hline $7440-39-3$ & Barium & $6.53 \mathrm{E} 1$ & ug/g dry & $1.49 \mathrm{E} 0$ & $2 / 02 / 11$ & 1B02001 & PNNL-AGG-ICP-AES \\
\hline HEIS No. & B273B3 & \multicolumn{3}{|c|}{ Lab ID: $\quad$ 1009008-08 } & & & \\
\hline $7440-39-3$ & Barium & $5.15 \mathrm{E} 1$ & ug/g dry & $1.70 \mathrm{E} 0$ & $2 / 02 / 11$ & $1 \mathrm{~B} 02001$ & PNNL-AGG-ICP-AES \\
\hline HEIS No. & B273B4 & \multicolumn{3}{|c|}{ Lab ID: $\quad 1009008-09$} & & & \\
\hline $7440-39-3$ & Barium & $6.17 \mathrm{E} 1$ & ug/g dry & $1.60 \mathrm{E} 0$ & $2 / 02 / 11$ & 1B02001 & PNNL-AGG-ICP-AES \\
\hline HEIS No. & B273B6 & \multicolumn{3}{|c|}{ Lab ID: $\quad 1009008-10$} & & & \\
\hline $7440-39-3$ & Barium & $1.36 \mathrm{E} 3$ & ug/g dry & $1.96 \mathrm{E} 0$ & $2 / 02 / 11$ & 1B02001 & PNNL-AGG-ICP-AES \\
\hline HEIS No. & B273B5 & \multicolumn{3}{|c|}{ Lab ID: $\quad$ 1009008-11 } & & & \\
\hline $7440-39-3$ & Barium & $1.03 \mathrm{E} 2$ & ug/g dry & $1.85 \mathrm{E} 0$ & $2 / 02 / 11$ & 1B02001 & PNNL-AGG-ICP-AES \\
\hline
\end{tabular}


RCRA Metals By PNNL-AGG-415/1:1 Water Extract

\begin{tabular}{|c|c|c|c|c|c|c|c|}
\hline CAS \# & Analyte & Results & Units & EQL & Analyzed & Batch & Method \\
\hline HEIS No. & B274K7 & \multicolumn{3}{|c|}{ Lab ID: $\quad$ 1009008-01 } & & & \\
\hline $14092-98-9$ & Chromium & $<3.23 \mathrm{E}-3$ & ug/g dry & $3.23 \mathrm{E}-3$ & $10 / 11 / 10$ & 0J11002 & PNNL-AGG-415 \\
\hline $7440-38-2$ & Arsenic & $9.25 \mathrm{E}-3$ & ug/g dry & $2.66 \mathrm{E}-3$ & $10 / 11 / 10$ & 0J11002 & PNNL-AGG-415 \\
\hline $14687-58-2$ & Selenium & $1.83 \mathrm{E}-2$ & ug/g dry & 7.43E-3 & $10 / 11 / 10$ & 0J11002 & PNNL-AGG-415 \\
\hline 14378-37-1 & Silver & $<2.94 \mathrm{E}-3$ & ug/g dry & $2.94 \mathrm{E}-3$ & $10 / 11 / 10$ & 0J11002 & PNNL-AGG-415 \\
\hline $14336-64-2$ & Cadmium & $<5.38 \mathrm{E}-4$ & ug/g dry & $5.38 \mathrm{E}-4$ & $10 / 11 / 10$ & 0J11002 & PNNL-AGG-415 \\
\hline $13966-28-4$ & Lead & $<1.09 \mathrm{E}-3$ & ug/g dry & $1.09 \mathrm{E}-3$ & $10 / 11 / 10$ & 0J11002 & PNNL-AGG-415 \\
\hline HEIS No. & B273B8 & \multicolumn{3}{|c|}{ Lab ID: $\quad 1009008-02$} & & & \\
\hline $14092-98-9$ & Chromium & $<3.44 \mathrm{E}-3$ & ug/g dry & $3.44 \mathrm{E}-3$ & $10 / 11 / 10$ & $0 \mathrm{~J} 11002$ & PNNL-AGG-415 \\
\hline $7440-38-2$ & Arsenic & $<2.83 \mathrm{E}-3$ & ug/g dry & $2.83 \mathrm{E}-3$ & $10 / 11 / 10$ & 0J11002 & PNNL-AGG-415 \\
\hline $14687-58-2$ & Selenium & $<7.90 \mathrm{E}-3$ & ug/g dry & $7.90 \mathrm{E}-3$ & $10 / 11 / 10$ & 0J11002 & PNNL-AGG-415 \\
\hline $14378-37-1$ & Silver & $<3.12 \mathrm{E}-3$ & ug/g dry & $3.12 \mathrm{E}-3$ & $10 / 11 / 10$ & $0 \mathrm{~J} 11002$ & PNNL-AGG-415 \\
\hline $14336-64-2$ & Cadmium & $<5.72 \mathrm{E}-4$ & ug/g dry & $5.72 \mathrm{E}-4$ & $10 / 11 / 10$ & 0J11002 & PNNL-AGG-415 \\
\hline $13966-28-4$ & Lead & $<1.16 \mathrm{E}-3$ & ug/g dry & $1.16 \mathrm{E}-3$ & $10 / 11 / 10$ & $0 \mathrm{~J} 11002$ & PNNL-AGG-415 \\
\hline HEIS No. & B27PY0 & \multicolumn{3}{|c|}{ Lab ID: $\quad 1009008-03$} & & & \\
\hline 14092-98-9 & Chromium & $<3.49 \mathrm{E}-3$ & ug/g dry & $3.49 \mathrm{E}-3$ & $10 / 11 / 10$ & 0J11002 & PNNL-AGG-415 \\
\hline $7440-38-2$ & Arsenic & $<2.87 \mathrm{E}-3$ & ug/g dry & $2.87 \mathrm{E}-3$ & $10 / 11 / 10$ & $0 \mathrm{~J} 11002$ & PNNL-AGG-415 \\
\hline $14687-58-2$ & Selenium & $<8.02 \mathrm{E}-3$ & ug/g dry & $8.02 \mathrm{E}-3$ & $10 / 11 / 10$ & $0 \mathrm{~J} 11002$ & PNNL-AGG-415 \\
\hline $14378-37-1$ & Silver & $<3.17 \mathrm{E}-3$ & ug/g dry & $3.17 \mathrm{E}-3$ & $10 / 11 / 10$ & $0 \mathrm{~J} 11002$ & PNNL-AGG-415 \\
\hline $14336-64-2$ & Cadmium & $<5.81 \mathrm{E}-4$ & ug/g dry & $5.81 \mathrm{E}-4$ & $10 / 11 / 10$ & 0J11002 & PNNL-AGG-415 \\
\hline $13966-28-4$ & Lead & $<1.18 \mathrm{E}-3$ & ug/g dry & $1.18 \mathrm{E}-3$ & $10 / 11 / 10$ & $0 \mathrm{~J} 11002$ & PNNL-AGG-415 \\
\hline HEIS No. & B274K6 & \multicolumn{3}{|c|}{ Lab ID: $\quad 1009008-04$} & & & \\
\hline $14092-98-9$ & Chromium & $<3.46 \mathrm{E}-3$ & ug/g dry & $3.46 \mathrm{E}-3$ & $10 / 11 / 10$ & $0 \mathrm{~J} 11002$ & PNNL-AGG-415 \\
\hline $7440-38-2$ & Arsenic & $<2.84 \mathrm{E}-3$ & ug/g dry & $2.84 \mathrm{E}-3$ & $10 / 11 / 10$ & $0 \mathrm{~J} 11002$ & PNNL-AGG-415 \\
\hline $14687-58-2$ & Selenium & $<7.94 \mathrm{E}-3$ & ug/g dry & $7.94 \mathrm{E}-3$ & $10 / 11 / 10$ & $0 \mathrm{~J} 11002$ & PNNL-AGG-415 \\
\hline $14378-37-1$ & Silver & $<3.14 \mathrm{E}-3$ & ug/g dry & $3.14 \mathrm{E}-3$ & $10 / 11 / 10$ & $0 \mathrm{~J} 11002$ & PNNL-AGG-415 \\
\hline $14336-64-2$ & Cadmium & $<5.75 \mathrm{E}-4$ & ug/g dry & $5.75 \mathrm{E}-4$ & $10 / 11 / 10$ & $0 \mathrm{~J} 11002$ & PNNL-AGG-415 \\
\hline $13966-28-4$ & Lead & $<1.17 \mathrm{E}-3$ & ug/g dry & $1.17 \mathrm{E}-3$ & $10 / 11 / 10$ & 0J11002 & PNNL-AGG-415 \\
\hline HEIS No. & B26TC1 & \multicolumn{3}{|c|}{ Lab ID: $1009008-05$} & & & \\
\hline 14092-98-9 & Chromium & $<3.45 \mathrm{E}-3$ & ug/g dry & $3.45 \mathrm{E}-3$ & $10 / 11 / 10$ & $0 \mathrm{~J} 11002$ & PNNL-AGG-415 \\
\hline $7440-38-2$ & Arsenic & $<2.84 \mathrm{E}-3$ & ug/g dry & $2.84 \mathrm{E}-3$ & $10 / 11 / 10$ & $0 \mathrm{~J} 11002$ & PNNL-AGG-415 \\
\hline $14687-58-2$ & Selenium & $<7.92 \mathrm{E}-3$ & ug/g dry & $7.92 \mathrm{E}-3$ & $10 / 11 / 10$ & 0J11002 & PNNL-AGG-415 \\
\hline $14378-37-1$ & Silver & $<3.13 \mathrm{E}-3$ & ug/g dry & $3.13 \mathrm{E}-3$ & $10 / 11 / 10$ & $0 \mathrm{~J} 11002$ & PNNL-AGG-415 \\
\hline $14336-64-2$ & Cadmium & $<5.74 \mathrm{E}-4$ & ug/g dry & $5.74 \mathrm{E}-4$ & $10 / 11 / 10$ & 0J11002 & PNNL-AGG-415 \\
\hline $13966-28-4$ & Lead & $<1.16 \mathrm{E}-3$ & ug/g dry & $1.16 \mathrm{E}-3$ & $10 / 11 / 10$ & $0 \mathrm{~J} 11002$ & PNNL-AGG-415 \\
\hline HEIS No. & B26TC2 & \multicolumn{3}{|c|}{ Lab ID: $\quad 1009008-06$} & & & \\
\hline 14092-98-9 & Chromium & $<3.59 \mathrm{E}-3$ & ug/g dry & $3.59 \mathrm{E}-3$ & $10 / 11 / 10$ & $0 \mathrm{~J} 11002$ & PNNL-AGG-415 \\
\hline $7440-38-2$ & Arsenic & $8.86 \mathrm{E}-3$ & ug/g dry & $2.96 \mathrm{E}-3$ & $10 / 11 / 10$ & $0 \mathrm{~J} 11002$ & PNNL-AGG-415 \\
\hline $14687-58-2$ & Selenium & $<8.25 \mathrm{E}-3$ & ug/g dry & $8.25 \mathrm{E}-3$ & $10 / 11 / 10$ & $0 \mathrm{~J} 11002$ & PNNL-AGG-415 \\
\hline $14378-37-1$ & Silver & $<3.26 \mathrm{E}-3$ & ug/g dry & $3.26 \mathrm{E}-3$ & $10 / 11 / 10$ & 0J11002 & PNNL-AGG-415 \\
\hline $14336-64-2$ & Cadmium & $<5.97 \mathrm{E}-4$ & ug/g dry & $5.97 \mathrm{E}-4$ & $10 / 11 / 10$ & $0 \mathrm{~J} 11002$ & PNNL-AGG-415 \\
\hline $13966-28-4$ & Lead & $<1.21 \mathrm{E}-3$ & ug/g dry & $1.21 \mathrm{E}-3$ & $10 / 11 / 10$ & 0J11002 & PNNL-AGG-415 \\
\hline HEIS No. & B273B2 & \multicolumn{3}{|c|}{ Lab ID: $\quad 1009008-07$} & & & \\
\hline $14092-98-9$ & Chromium & $<3.44 \mathrm{E}-3$ & ug/g dry & $3.44 \mathrm{E}-3$ & $1 / 17 / 11$ & $1 \mathrm{~A} 17001$ & PNNL-AGG-415 \\
\hline $7440-38-2$ & Arsenic & $2.15 \mathrm{E}-2$ & ug/g dry & $1.42 \mathrm{E}-2$ & $1 / 21 / 11$ & 1A17001 & PNNL-AGG-415 \\
\hline $14687-58-2$ & Selenium & $<7.91 \mathrm{E}-3$ & ug/g dry & $7.91 \mathrm{E}-3$ & $1 / 17 / 11$ & $1 \mathrm{~A} 17001$ & PNNL-AGG-415 \\
\hline $14378-37-1$ & Silver & $<3.13 \mathrm{E}-3$ & ug/g dry & $3.13 \mathrm{E}-3$ & $1 / 17 / 11$ & $1 \mathrm{~A} 17001$ & PNNL-AGG-415 \\
\hline $14336-64-2$ & Cadmium & $<5.73 \mathrm{E}-4$ & ug/g dry & $5.73 \mathrm{E}-4$ & $1 / 17 / 11$ & 1A17001 & PNNL-AGG-415 \\
\hline $13966-28-4$ & Lead & $<1.16 \mathrm{E}-3$ & ug/g dry & $1.16 \mathrm{E}-3$ & $1 / 17 / 11$ & $1 \mathrm{~A} 17001$ & PNNL-AGG-415 \\
\hline
\end{tabular}


RCRA Metals By PNNL-AGG-415/1:1 Water Extract

\begin{tabular}{|c|c|c|c|c|c|c|c|}
\hline CAS \# & Analyte & Results & Units & EQL & Analyzed & Batch & Method \\
\hline HEIS No. & B273B3 & \multicolumn{3}{|c|}{ Lab ID: $\quad 1009008-08$} & & & \\
\hline 14092-98-9 & Chromium & $<3.45 \mathrm{E}-3$ & ug/g dry & $3.45 \mathrm{E}-3$ & $1 / 17 / 11$ & 1A17001 & PNNL-AGG-415 \\
\hline $7440-38-2$ & Arsenic & $<1.42 \mathrm{E}-2$ & ug/g dry & $1.42 \mathrm{E}-2$ & $1 / 21 / 11$ & $1 \mathrm{~A} 17001$ & PNNL-AGG-415 \\
\hline $14687-58-2$ & Selenium & $<7.92 \mathrm{E}-3$ & ug/g dry & $7.92 \mathrm{E}-3$ & $1 / 17 / 11$ & $1 \mathrm{~A} 17001$ & PNNL-AGG-415 \\
\hline $14378-37-1$ & Silver & $<3.13 \mathrm{E}-3$ & ug/g dry & $3.13 \mathrm{E}-3$ & $1 / 17 / 11$ & 1A17001 & PNNL-AGG-415 \\
\hline $14336-64-2$ & Cadmium & $<5.74 \mathrm{E}-4$ & ug/g dry & $5.74 \mathrm{E}-4$ & $1 / 17 / 11$ & $1 \mathrm{~A} 17001$ & PNNL-AGG-415 \\
\hline $13966-28-4$ & Lead & $<1.16 \mathrm{E}-3$ & ug/g dry & $1.16 \mathrm{E}-3$ & $1 / 17 / 11$ & $1 \mathrm{~A} 17001$ & PNNL-AGG-415 \\
\hline HEIS No. & B273B4 & \multicolumn{3}{|c|}{ Lab ID: $\quad 1009008-09$} & & & \\
\hline $14092-98-9$ & Chromium & $<3.45 \mathrm{E}-3$ & ug/g dry & $3.45 \mathrm{E}-3$ & $1 / 17 / 11$ & $1 \mathrm{~A} 17001$ & PNNL-AGG-415 \\
\hline $7440-38-2$ & Arsenic & $2.70 \mathrm{E}-2$ & ug/g dry & $1.42 \mathrm{E}-2$ & $1 / 21 / 11$ & $1 \mathrm{~A} 17001$ & PNNL-AGG-415 \\
\hline $14687-58-2$ & Selenium & $<7.93 \mathrm{E}-3$ & ug/g dry & 7.93E-3 & $1 / 17 / 11$ & $1 \mathrm{~A} 17001$ & PNNL-AGG-415 \\
\hline $14378-37-1$ & Silver & $<3.13 \mathrm{E}-3$ & ug/g dry & $3.13 \mathrm{E}-3$ & $1 / 17 / 11$ & $1 \mathrm{~A} 17001$ & PNNL-AGG-415 \\
\hline $14336-64-2$ & Cadmium & $<5.74 \mathrm{E}-4$ & ug/g dry & $5.74 \mathrm{E}-4$ & $1 / 17 / 11$ & 1A17001 & PNNL-AGG-415 \\
\hline $13966-28-4$ & Lead & $<1.16 \mathrm{E}-3$ & ug/g dry & $1.16 \mathrm{E}-3$ & $1 / 17 / 11$ & $1 \mathrm{~A} 17001$ & PNNL-AGG-415 \\
\hline HEIS No. & B273B6 & \multicolumn{3}{|c|}{ Lab ID: $\quad 1009008-10$} & & & \\
\hline $14092-98-9$ & Chromium & $2.15 \mathrm{E}-2$ & ug/g dry & $3.42 \mathrm{E}-3$ & $1 / 17 / 11$ & $1 \mathrm{~A} 17001$ & PNNL-AGG-415 \\
\hline $7440-38-2$ & Arsenic & $3.97 \mathrm{E}-2$ & ug/g dry & $1.41 \mathrm{E}-2$ & $1 / 21 / 11$ & $1 \mathrm{~A} 17001$ & PNNL-AGG-415 \\
\hline $14687-58-2$ & Selenium & $<7.86 \mathrm{E}-3$ & ug/g dry & $7.86 \mathrm{E}-3$ & $1 / 17 / 11$ & 1A17001 & PNNL-AGG-415 \\
\hline $14378-37-1$ & Silver & $<3.11 \mathrm{E}-3$ & ug/g dry & $3.11 \mathrm{E}-3$ & $1 / 17 / 11$ & $1 \mathrm{~A} 17001$ & PNNL-AGG-415 \\
\hline $14336-64-2$ & Cadmium & $<5.69 \mathrm{E}-4$ & ug/g dry & $5.69 \mathrm{E}-4$ & $1 / 17 / 11$ & $1 \mathrm{~A} 17001$ & PNNL-AGG-415 \\
\hline $13966-28-4$ & Lead & $<1.15 \mathrm{E}-3$ & ug/g dry & $1.15 \mathrm{E}-3$ & $1 / 17 / 11$ & $1 \mathrm{~A} 17001$ & PNNL-AGG-415 \\
\hline HEIS No. & B273B5 & \multicolumn{3}{|c|}{ Lab ID: $\quad 1009008-11$} & & & \\
\hline $14092-98-9$ & Chromium & $<3.55 \mathrm{E}-3$ & ug/g dry & $3.55 \mathrm{E}-3$ & $1 / 17 / 11$ & $1 \mathrm{~A} 17001$ & PNNL-AGG-415 \\
\hline $7440-38-2$ & Arsenic & $1.89 \mathrm{E}-2$ & ug/g dry & $1.46 \mathrm{E}-2$ & $1 / 21 / 11$ & $1 \mathrm{~A} 17001$ & PNNL-AGG-415 \\
\hline $14687-58-2$ & Selenium & $<8.17 \mathrm{E}-3$ & ug/g dry & $8.17 \mathrm{E}-3$ & $1 / 17 / 11$ & $1 \mathrm{~A} 17001$ & PNNL-AGG-415 \\
\hline $14378-37-1$ & Silver & $<3.23 \mathrm{E}-3$ & ug/g dry & $3.23 \mathrm{E}-3$ & $1 / 17 / 11$ & 1A17001 & PNNL-AGG-415 \\
\hline $14336-64-2$ & Cadmium & $<5.91 \mathrm{E}-4$ & ug/g dry & $5.91 \mathrm{E}-4$ & $1 / 17 / 11$ & 1A17001 & PNNL-AGG-415 \\
\hline $13966-28-4$ & Lead & $<1.20 \mathrm{E}-3$ & ug/g dry & $1.20 \mathrm{E}-3$ & $1 / 17 / 11$ & $1 \mathrm{~A} 17001$ & PNNL-AGG-415 \\
\hline
\end{tabular}


RCRA Metals By PNNL-AGG-415/1:2.5 Water Extract

\begin{tabular}{|c|c|c|c|c|c|c|c|}
\hline CAS \# & Analyte & Results & Units & EQL & Analyzed & Batch & Method \\
\hline HEIS No. & B274K7 & \multicolumn{3}{|c|}{ Lab ID: $\quad$ 1009008-01 } & & & \\
\hline $14092-98-9$ & Chromium & $<8.22 \mathrm{E}-3$ & ug/g dry & $8.22 \mathrm{E}-3$ & $12 / 06 / 10$ & 0J11003 & PNNL-AGG-415 \\
\hline $7440-38-2$ & Arsenic & $8.83 \mathrm{E}-3$ & ug/g dry & $6.77 \mathrm{E}-3$ & $12 / 06 / 10$ & 0J11003 & PNNL-AGG-415 \\
\hline $14687-58-2$ & Selenium & $<1.89 \mathrm{E}-2$ & ug/g dry & $1.89 \mathrm{E}-2$ & $12 / 06 / 10$ & 0J11003 & PNNL-AGG-415 \\
\hline 14378-37-1 & Silver & $<7.47 \mathrm{E}-3$ & ug/g dry & $7.47 \mathrm{E}-3$ & $12 / 06 / 10$ & 0J11003 & PNNL-AGG-415 \\
\hline $14336-64-2$ & Cadmium & $<1.37 \mathrm{E}-3$ & ug/g dry & $1.37 \mathrm{E}-3$ & $12 / 06 / 10$ & 0J11003 & PNNL-AGG-415 \\
\hline $13966-28-4$ & Lead & $<2.77 \mathrm{E}-3$ & ug/g dry & $2.77 \mathrm{E}-3$ & $12 / 06 / 10$ & 0J11003 & PNNL-AGG-415 \\
\hline HEIS No. & B273B8 & \multicolumn{3}{|c|}{ Lab ID: $\quad 1009008-02$} & & & \\
\hline 14092-98-9 & Chromium & $<8.58 \mathrm{E}-3$ & ug/g dry & $8.58 \mathrm{E}-3$ & $12 / 06 / 10$ & 0J11003 & PNNL-AGG-415 \\
\hline $7440-38-2$ & Arsenic & $<7.07 \mathrm{E}-3$ & ug/g dry & $7.07 \mathrm{E}-3$ & $12 / 06 / 10$ & 0J11003 & PNNL-AGG-415 \\
\hline $14687-58-2$ & Selenium & $<1.97 \mathrm{E}-2$ & ug/g dry & $1.97 \mathrm{E}-2$ & $12 / 06 / 10$ & 0J11003 & PNNL-AGG-415 \\
\hline $14378-37-1$ & Silver & $<7.79 \mathrm{E}-3$ & ug/g dry & $7.79 \mathrm{E}-3$ & $12 / 06 / 10$ & $0 \mathrm{~J} 11003$ & PNNL-AGG-415 \\
\hline $14336-64-2$ & Cadmium & $<1.43 \mathrm{E}-3$ & ug/g dry & $1.43 \mathrm{E}-3$ & $12 / 06 / 10$ & $0 \mathrm{~J} 11003$ & PNNL-AGG-415 \\
\hline $13966-28-4$ & Lead & $<2.90 \mathrm{E}-3$ & ug/g dry & $2.90 \mathrm{E}-3$ & $12 / 06 / 10$ & $0 \mathrm{~J} 11003$ & PNNL-AGG-415 \\
\hline HEIS No. & B27PY0 & \multicolumn{3}{|c|}{ Lab ID: $\quad 1009008-03$} & & & \\
\hline 14092-98-9 & Chromium & $<8.62 \mathrm{E}-3$ & ug/g dry & $8.62 \mathrm{E}-3$ & $12 / 06 / 10$ & $0 \mathrm{~J} 11003$ & PNNL-AGG-415 \\
\hline $7440-38-2$ & Arsenic & $<7.09 \mathrm{E}-3$ & ug/g dry & $7.09 \mathrm{E}-3$ & $12 / 06 / 10$ & $0 \mathrm{~J} 11003$ & PNNL-AGG-415 \\
\hline $14687-58-2$ & Selenium & $<1.98 \mathrm{E}-2$ & ug/g dry & $1.98 \mathrm{E}-2$ & $12 / 06 / 10$ & $0 \mathrm{~J} 11003$ & PNNL-AGG-415 \\
\hline $14378-37-1$ & Silver & $<7.82 \mathrm{E}-3$ & ug/g dry & $7.82 \mathrm{E}-3$ & $12 / 06 / 10$ & $0 \mathrm{~J} 11003$ & PNNL-AGG-415 \\
\hline $14336-64-2$ & Cadmium & $<1.43 \mathrm{E}-3$ & ug/g dry & $1.43 \mathrm{E}-3$ & $12 / 06 / 10$ & $0 \mathrm{~J} 11003$ & PNNL-AGG-415 \\
\hline $13966-28-4$ & Lead & $<2.91 \mathrm{E}-3$ & ug/g dry & $2.91 \mathrm{E}-3$ & $12 / 06 / 10$ & $0 \mathrm{~J} 11003$ & PNNL-AGG-415 \\
\hline HEIS No. & B274K6 & \multicolumn{3}{|c|}{ Lab ID: $\quad 1009008-04$} & & & \\
\hline $14092-98-9$ & Chromium & $<9.20 \mathrm{E}-3$ & ug/g dry & $9.20 \mathrm{E}-3$ & $12 / 06 / 10$ & $0 \mathrm{~J} 11003$ & PNNL-AGG-415 \\
\hline $7440-38-2$ & Arsenic & $<7.57 \mathrm{E}-3$ & ug/g dry & $7.57 \mathrm{E}-3$ & $12 / 06 / 10$ & 0J11003 & PNNL-AGG-415 \\
\hline $14687-58-2$ & Selenium & $<2.11 \mathrm{E}-2$ & ug/g dry & $2.11 \mathrm{E}-2$ & $12 / 06 / 10$ & $0 \mathrm{~J} 11003$ & PNNL-AGG-415 \\
\hline $14378-37-1$ & Silver & $<8.35 \mathrm{E}-3$ & ug/g dry & $8.35 \mathrm{E}-3$ & $12 / 06 / 10$ & $0 \mathrm{~J} 11003$ & PNNL-AGG-415 \\
\hline $14336-64-2$ & Cadmium & $<1.53 \mathrm{E}-3$ & ug/g dry & $1.53 \mathrm{E}-3$ & $12 / 06 / 10$ & $0 \mathrm{~J} 11003$ & PNNL-AGG-415 \\
\hline $13966-28-4$ & Lead & $<3.10 \mathrm{E}-3$ & ug/g dry & $3.10 \mathrm{E}-3$ & $12 / 06 / 10$ & $0 \mathrm{~J} 11003$ & PNNL-AGG-415 \\
\hline HEIS No. & B26TC1 & \multicolumn{3}{|c|}{ Lab ID: $1009008-05$} & & & \\
\hline 14092-98-9 & Chromium & $<8.57 \mathrm{E}-3$ & ug/g dry & $8.57 \mathrm{E}-3$ & $12 / 06 / 10$ & 0J11003 & PNNL-AGG-415 \\
\hline $7440-38-2$ & Arsenic & $<7.06 \mathrm{E}-3$ & ug/g dry & $7.06 \mathrm{E}-3$ & $12 / 06 / 10$ & $0 \mathrm{~J} 11003$ & PNNL-AGG-415 \\
\hline $14687-58-2$ & Selenium & $<1.97 \mathrm{E}-2$ & ug/g dry & $1.97 \mathrm{E}-2$ & $12 / 06 / 10$ & 0J11003 & PNNL-AGG-415 \\
\hline $14378-37-1$ & Silver & $<7.78 \mathrm{E}-3$ & ug/g dry & $7.78 \mathrm{E}-3$ & $12 / 06 / 10$ & $0 \mathrm{~J} 11003$ & PNNL-AGG-415 \\
\hline $14336-64-2$ & Cadmium & $<1.43 \mathrm{E}-3$ & ug/g dry & $1.43 \mathrm{E}-3$ & $12 / 06 / 10$ & $0 \mathrm{~J} 11003$ & PNNL-AGG-415 \\
\hline $13966-28-4$ & Lead & $<2.89 \mathrm{E}-3$ & ug/g dry & $2.89 \mathrm{E}-3$ & $12 / 06 / 10$ & $0 \mathrm{~J} 11003$ & PNNL-AGG-415 \\
\hline HEIS No. & B26TC2 & \multicolumn{3}{|c|}{ Lab ID: $\quad 1009008-06$} & & & \\
\hline 14092-98-9 & Chromium & $<8.55 \mathrm{E}-3$ & ug/g dry & $8.55 \mathrm{E}-3$ & $12 / 06 / 10$ & 0J11003 & PNNL-AGG-415 \\
\hline $7440-38-2$ & Arsenic & $1.28 \mathrm{E}-2$ & ug/g dry & 7.04E-3 & $12 / 06 / 10$ & $0 \mathrm{~J} 11003$ & PNNL-AGG-415 \\
\hline $14687-58-2$ & Selenium & $<1.97 \mathrm{E}-2$ & ug/g dry & $1.97 \mathrm{E}-2$ & $12 / 06 / 10$ & $0 \mathrm{~J} 11003$ & PNNL-AGG-415 \\
\hline $14378-37-1$ & Silver & $<7.77 \mathrm{E}-3$ & ug/g dry & $7.77 \mathrm{E}-3$ & $12 / 06 / 10$ & 0J11003 & PNNL-AGG-415 \\
\hline $14336-64-2$ & Cadmium & $<1.42 \mathrm{E}-3$ & ug/g dry & $1.42 \mathrm{E}-3$ & $12 / 06 / 10$ & $0 \mathrm{~J} 11003$ & PNNL-AGG-415 \\
\hline $13966-28-4$ & Lead & $<2.89 \mathrm{E}-3$ & ug/g dry & $2.89 \mathrm{E}-3$ & $12 / 06 / 10$ & 0J11003 & PNNL-AGG-415 \\
\hline HEIS No. & B273B2 & \multicolumn{3}{|c|}{ Lab ID: $\quad 1009008-07$} & & & \\
\hline $14092-98-9$ & Chromium & $<8.53 \mathrm{E}-3$ & ug/g dry & $8.53 \mathrm{E}-3$ & $1 / 17 / 11$ & $1 \mathrm{~A} 17002$ & PNNL-AGG-415 \\
\hline $7440-38-2$ & Arsenic & $4.34 \mathrm{E}-2$ & ug/g dry & $3.51 \mathrm{E}-2$ & $1 / 21 / 11$ & 1A17002 & PNNL-AGG-415 \\
\hline $14687-58-2$ & Selenium & $<1.96 \mathrm{E}-2$ & ug/g dry & $1.96 \mathrm{E}-2$ & $1 / 17 / 11$ & $1 \mathrm{~A} 17002$ & PNNL-AGG-415 \\
\hline $14378-37-1$ & Silver & $<7.74 \mathrm{E}-3$ & ug/g dry & $7.74 \mathrm{E}-3$ & $1 / 17 / 11$ & $1 \mathrm{~A} 17002$ & PNNL-AGG-415 \\
\hline $14336-64-2$ & Cadmium & $<1.42 \mathrm{E}-3$ & ug/g dry & $1.42 \mathrm{E}-3$ & $1 / 17 / 11$ & 1A 17002 & PNNL-AGG-415 \\
\hline $13966-28-4$ & Lead & $<2.88 \mathrm{E}-3$ & ug/g dry & $2.88 \mathrm{E}-3$ & $1 / 17 / 11$ & 1A17002 & PNNL-AGG-415 \\
\hline
\end{tabular}


RCRA Metals By PNNL-AGG-415/1:2.5 Water Extract

\begin{tabular}{|c|c|c|c|c|c|c|c|}
\hline CAS \# & Analyte & Results & Units & EQL & Analyzed & Batch & Method \\
\hline HEIS No. & B273B3 & \multicolumn{3}{|c|}{ Lab ID: $\quad 1009008-08$} & & & \\
\hline 14092-98-9 & Chromium & $<8.54 \mathrm{E}-3$ & ug/g dry & $8.54 \mathrm{E}-3$ & $1 / 17 / 11$ & $1 \mathrm{~A} 17002$ & PNNL-AGG-415 \\
\hline $7440-38-2$ & Arsenic & $3.65 \mathrm{E}-2$ & ug/g dry & $3.52 \mathrm{E}-2$ & $1 / 21 / 11$ & 1A17002 & PNNL-AGG-415 \\
\hline $14687-58-2$ & Selenium & $<1.96 \mathrm{E}-2$ & ug/g dry & $1.96 \mathrm{E}-2$ & $1 / 17 / 11$ & $1 \mathrm{~A} 17002$ & PNNL-AGG-415 \\
\hline $14378-37-1$ & Silver & $<7.76 \mathrm{E}-3$ & ug/g dry & $7.76 \mathrm{E}-3$ & $1 / 17 / 11$ & $1 \mathrm{~A} 17002$ & PNNL-AGG-415 \\
\hline $14336-64-2$ & Cadmium & $<1.42 \mathrm{E}-3$ & ug/g dry & $1.42 \mathrm{E}-3$ & $1 / 17 / 11$ & $1 \mathrm{~A} 17002$ & PNNL-AGG-415 \\
\hline $13966-28-4$ & Lead & $<2.88 \mathrm{E}-3$ & ug/g dry & $2.88 \mathrm{E}-3$ & $1 / 17 / 11$ & $1 \mathrm{~A} 17002$ & PNNL-AGG-415 \\
\hline HEIS No. & B273B4 & \multicolumn{3}{|c|}{ Lab ID: $\quad 1009008-09$} & & & \\
\hline $14092-98-9$ & Chromium & $<8.57 \mathrm{E}-3$ & ug/g dry & $8.57 \mathrm{E}-3$ & $1 / 17 / 11$ & $1 \mathrm{~A} 17002$ & PNNL-AGG-415 \\
\hline $7440-38-2$ & Arsenic & $5.86 \mathrm{E}-2$ & ug/g dry & $3.53 \mathrm{E}-2$ & $1 / 21 / 11$ & $1 \mathrm{~A} 17002$ & PNNL-AGG-415 \\
\hline $14687-58-2$ & Selenium & $<1.97 \mathrm{E}-2$ & ug/g dry & $1.97 \mathrm{E}-2$ & $1 / 17 / 11$ & $1 \mathrm{~A} 17002$ & PNNL-AGG-415 \\
\hline $14378-37-1$ & Silver & $<7.78 \mathrm{E}-3$ & ug/g dry & $7.78 \mathrm{E}-3$ & $1 / 17 / 11$ & $1 \mathrm{~A} 17002$ & PNNL-AGG-415 \\
\hline $14336-64-2$ & Cadmium & $<1.43 \mathrm{E}-3$ & ug/g dry & $1.43 \mathrm{E}-3$ & $1 / 17 / 11$ & $1 \mathrm{~A} 17002$ & PNNL-AGG-415 \\
\hline $13966-28-4$ & Lead & $<2.89 \mathrm{E}-3$ & ug/g dry & $2.89 \mathrm{E}-3$ & $1 / 17 / 11$ & $1 \mathrm{~A} 17002$ & PNNL-AGG-415 \\
\hline HEIS No. & B273B6 & \multicolumn{3}{|c|}{ Lab ID: $\quad 1009008-10$} & & & \\
\hline $14092-98-9$ & Chromium & $2.13 \mathrm{E}-2$ & ug/g dry & $8.58 \mathrm{E}-3$ & $1 / 17 / 11$ & $1 \mathrm{~A} 17002$ & PNNL-AGG-415 \\
\hline $7440-38-2$ & Arsenic & $1.05 \mathrm{E}-1$ & ug/g dry & $3.53 \mathrm{E}-2$ & $1 / 21 / 11$ & $1 \mathrm{~A} 17002$ & PNNL-AGG-415 \\
\hline $14687-58-2$ & Selenium & $<1.97 \mathrm{E}-2$ & ug/g dry & $1.97 \mathrm{E}-2$ & $1 / 17 / 11$ & $1 \mathrm{~A} 17002$ & PNNL-AGG-415 \\
\hline $14378-37-1$ & Silver & $<7.79 \mathrm{E}-3$ & ug/g dry & $7.79 \mathrm{E}-3$ & $1 / 17 / 11$ & $1 \mathrm{~A} 17002$ & PNNL-AGG-415 \\
\hline $14336-64-2$ & Cadmium & $<1.43 \mathrm{E}-3$ & ug/g dry & $1.43 \mathrm{E}-3$ & $1 / 17 / 11$ & $1 \mathrm{~A} 17002$ & PNNL-AGG-415 \\
\hline $13966-28-4$ & Lead & $<2.90 \mathrm{E}-3$ & ug/g dry & $2.90 \mathrm{E}-3$ & $1 / 17 / 11$ & $1 \mathrm{~A} 17002$ & PNNL-AGG-415 \\
\hline HEIS No. & B273B5 & \multicolumn{3}{|c|}{ Lab ID: $\quad 1009008-11$} & & & \\
\hline $14092-98-9$ & Chromium & $<8.61 \mathrm{E}-3$ & ug/g dry & $8.61 \mathrm{E}-3$ & $1 / 17 / 11$ & $1 \mathrm{~A} 17002$ & PNNL-AGG-415 \\
\hline $7440-38-2$ & Arsenic & $<3.54 \mathrm{E}-2$ & ug/g dry & $3.54 \mathrm{E}-2$ & $1 / 21 / 11$ & $1 \mathrm{~A} 17002$ & PNNL-AGG-415 \\
\hline $14687-58-2$ & Selenium & $<1.98 \mathrm{E}-2$ & ug/g dry & $1.98 \mathrm{E}-2$ & $1 / 17 / 11$ & $1 \mathrm{~A} 17002$ & PNNL-AGG-415 \\
\hline $14378-37-1$ & Silver & $<7.81 \mathrm{E}-3$ & ug/g dry & $7.81 \mathrm{E}-3$ & $1 / 17 / 11$ & 1A17002 & PNNL-AGG-415 \\
\hline $14336-64-2$ & Cadmium & $<1.43 \mathrm{E}-3$ & ug/g dry & $1.43 \mathrm{E}-3$ & $1 / 17 / 11$ & 1A17002 & PNNL-AGG-415 \\
\hline $13966-28-4$ & Lead & $<2.90 \mathrm{E}-3$ & ug/g dry & $2.90 \mathrm{E}-3$ & $1 / 17 / 11$ & $1 \mathrm{~A} 17002$ & PNNL-AGG-415 \\
\hline
\end{tabular}


RCRA Metals By PNNL-AGG-415/1:5 Water Extract

\begin{tabular}{|c|c|c|c|c|c|c|c|}
\hline CAS \# & Analyte & Results & Units & EQL & Analyzed & Batch & Method \\
\hline HEIS No. & B274K7 & \multicolumn{3}{|c|}{ Lab ID: $\quad$ 1009008-01 } & & & \\
\hline $14092-98-9$ & Chromium & $<1.65 \mathrm{E}-2$ & ug/g dry & $1.65 \mathrm{E}-2$ & $12 / 06 / 10$ & 0J11004 & PNNL-AGG-415 \\
\hline $7440-38-2$ & Arsenic & $<1.36 \mathrm{E}-2$ & ug/g dry & $1.36 \mathrm{E}-2$ & $12 / 06 / 10$ & 0J11004 & PNNL-AGG-415 \\
\hline $14687-58-2$ & Selenium & $<3.80 \mathrm{E}-2$ & ug/g dry & $3.80 \mathrm{E}-2$ & $12 / 06 / 10$ & 0J11004 & PNNL-AGG-415 \\
\hline 14378-37-1 & Silver & $<1.50 \mathrm{E}-2$ & ug/g dry & $1.50 \mathrm{E}-2$ & $12 / 06 / 10$ & 0J11004 & PNNL-AGG-415 \\
\hline $14336-64-2$ & Cadmium & $<2.75 \mathrm{E}-3$ & ug/g dry & $2.75 \mathrm{E}-3$ & $12 / 06 / 10$ & 0J11004 & PNNL-AGG-415 \\
\hline $13966-28-4$ & Lead & $<5.58 \mathrm{E}-3$ & ug/g dry & $5.58 \mathrm{E}-3$ & $12 / 06 / 10$ & 0J11004 & PNNL-AGG-415 \\
\hline HEIS No. & B273B8 & \multicolumn{3}{|c|}{ Lab ID: $\quad 1009008-02$} & & & \\
\hline 14092-98-9 & Chromium & $<1.70 \mathrm{E}-2$ & ug/g dry & $1.70 \mathrm{E}-2$ & $12 / 06 / 10$ & $0 \mathrm{~J} 11004$ & PNNL-AGG-415 \\
\hline $7440-38-2$ & Arsenic & $<1.40 \mathrm{E}-2$ & ug/g dry & $1.40 \mathrm{E}-2$ & $12 / 06 / 10$ & 0J11004 & PNNL-AGG-415 \\
\hline $14687-58-2$ & Selenium & $<3.92 \mathrm{E}-2$ & ug/g dry & $3.92 \mathrm{E}-2$ & $12 / 06 / 10$ & 0J11004 & PNNL-AGG-415 \\
\hline $14378-37-1$ & Silver & $<1.55 \mathrm{E}-2$ & ug/g dry & $1.55 \mathrm{E}-2$ & $12 / 06 / 10$ & $0 \mathrm{~J} 11004$ & PNNL-AGG-415 \\
\hline $14336-64-2$ & Cadmium & $<2.84 \mathrm{E}-3$ & ug/g dry & $2.84 \mathrm{E}-3$ & $12 / 06 / 10$ & $0 \mathrm{~J} 11004$ & PNNL-AGG-415 \\
\hline $13966-28-4$ & Lead & $<5.75 \mathrm{E}-3$ & ug/g dry & $5.75 \mathrm{E}-3$ & $12 / 06 / 10$ & $0 \mathrm{~J} 11004$ & PNNL-AGG-415 \\
\hline HEIS No. & B27PY0 & \multicolumn{3}{|c|}{ Lab ID: $\quad 1009008-03$} & & & \\
\hline 14092-98-9 & Chromium & $<1.58 \mathrm{E}-2$ & ug/g dry & $1.58 \mathrm{E}-2$ & $12 / 06 / 10$ & 0J11004 & PNNL-AGG-415 \\
\hline $7440-38-2$ & Arsenic & $<1.30 \mathrm{E}-2$ & ug/g dry & $1.30 \mathrm{E}-2$ & $12 / 06 / 10$ & $0 \mathrm{~J} 11004$ & PNNL-AGG-415 \\
\hline $14687-58-2$ & Selenium & $<3.64 \mathrm{E}-2$ & ug/g dry & $3.64 \mathrm{E}-2$ & $12 / 06 / 10$ & $0 \mathrm{~J} 11004$ & PNNL-AGG-415 \\
\hline $14378-37-1$ & Silver & $<1.44 \mathrm{E}-2$ & ug/g dry & $1.44 \mathrm{E}-2$ & $12 / 06 / 10$ & 0J11004 & PNNL-AGG-415 \\
\hline $14336-64-2$ & Cadmium & $<2.63 \mathrm{E}-3$ & ug/g dry & $2.63 \mathrm{E}-3$ & $12 / 06 / 10$ & 0J11004 & PNNL-AGG-415 \\
\hline $13966-28-4$ & Lead & $<5.34 \mathrm{E}-3$ & ug/g dry & $5.34 \mathrm{E}-3$ & $12 / 06 / 10$ & $0 \mathrm{~J} 11004$ & PNNL-AGG-415 \\
\hline HEIS No. & B274K6 & \multicolumn{3}{|c|}{ Lab ID: $\quad 1009008-04$} & & & \\
\hline $14092-98-9$ & Chromium & $<1.72 \mathrm{E}-2$ & ug/g dry & $1.72 \mathrm{E}-2$ & $12 / 06 / 10$ & $0 \mathrm{~J} 11004$ & PNNL-AGG-415 \\
\hline $7440-38-2$ & Arsenic & $<1.42 \mathrm{E}-2$ & ug/g dry & $1.42 \mathrm{E}-2$ & $12 / 06 / 10$ & 0J11004 & PNNL-AGG-415 \\
\hline $14687-58-2$ & Selenium & $<3.95 \mathrm{E}-2$ & ug/g dry & $3.95 \mathrm{E}-2$ & $12 / 06 / 10$ & $0 J 11004$ & PNNL-AGG-415 \\
\hline $14378-37-1$ & Silver & $<1.56 \mathrm{E}-2$ & ug/g dry & $1.56 \mathrm{E}-2$ & $12 / 06 / 10$ & $0 \mathrm{~J} 11004$ & PNNL-AGG-415 \\
\hline $14336-64-2$ & Cadmium & $<2.86 \mathrm{E}-3$ & ug/g dry & $2.86 \mathrm{E}-3$ & $12 / 06 / 10$ & $0 \mathrm{~J} 11004$ & PNNL-AGG-415 \\
\hline $13966-28-4$ & Lead & $<5.80 \mathrm{E}-3$ & ug/g dry & $5.80 \mathrm{E}-3$ & $12 / 06 / 10$ & 0J11004 & PNNL-AGG-415 \\
\hline HEIS No. & B26TC1 & \multicolumn{3}{|c|}{ Lab ID: $1009008-05$} & & & \\
\hline 14092-98-9 & Chromium & $<1.69 \mathrm{E}-2$ & ug/g dry & $1.69 \mathrm{E}-2$ & $12 / 06 / 10$ & 0J11004 & PNNL-AGG-415 \\
\hline $7440-38-2$ & Arsenic & $<1.39 \mathrm{E}-2$ & ug/g dry & $1.39 \mathrm{E}-2$ & $12 / 06 / 10$ & $0 \mathrm{~J} 11004$ & PNNL-AGG-415 \\
\hline $14687-58-2$ & Selenium & $<3.89 \mathrm{E}-2$ & ug/g dry & $3.89 \mathrm{E}-2$ & $12 / 06 / 10$ & 0J11004 & PNNL-AGG-415 \\
\hline $14378-37-1$ & Silver & $<1.54 \mathrm{E}-2$ & ug/g dry & $1.54 \mathrm{E}-2$ & $12 / 06 / 10$ & $0 \mathrm{~J} 11004$ & PNNL-AGG-415 \\
\hline $14336-64-2$ & Cadmium & $<2.82 \mathrm{E}-3$ & ug/g dry & $2.82 \mathrm{E}-3$ & $12 / 06 / 10$ & 0J11004 & PNNL-AGG-415 \\
\hline $13966-28-4$ & Lead & $<5.72 \mathrm{E}-3$ & ug/g dry & $5.72 \mathrm{E}-3$ & $12 / 06 / 10$ & $0 \mathrm{~J} 11004$ & PNNL-AGG-415 \\
\hline HEIS No. & B26TC2 & \multicolumn{3}{|c|}{ Lab ID: $\quad 1009008-06$} & & & \\
\hline 14092-98-9 & Chromium & $<1.72 \mathrm{E}-2$ & ug/g dry & $1.72 \mathrm{E}-2$ & $12 / 06 / 10$ & 0J11004 & PNNL-AGG-415 \\
\hline $7440-38-2$ & Arsenic & $2.00 \mathrm{E}-2$ & ug/g dry & $1.41 \mathrm{E}-2$ & $12 / 06 / 10$ & $0 \mathrm{~J} 11004$ & PNNL-AGG-415 \\
\hline $14687-58-2$ & Selenium & $<3.95 \mathrm{E}-2$ & ug/g dry & $3.95 \mathrm{E}-2$ & $12 / 06 / 10$ & $0 \mathrm{~J} 11004$ & PNNL-AGG-415 \\
\hline $14378-37-1$ & Silver & $<1.56 \mathrm{E}-2$ & ug/g dry & $1.56 \mathrm{E}-2$ & $12 / 06 / 10$ & 0J11004 & PNNL-AGG-415 \\
\hline $14336-64-2$ & Cadmium & $<2.86 \mathrm{E}-3$ & ug/g dry & $2.86 \mathrm{E}-3$ & $12 / 06 / 10$ & $0 \mathrm{~J} 11004$ & PNNL-AGG-415 \\
\hline $13966-28-4$ & Lead & $<5.80 \mathrm{E}-3$ & ug/g dry & $5.80 \mathrm{E}-3$ & $12 / 06 / 10$ & 0J11004 & PNNL-AGG-415 \\
\hline HEIS No. & B273B2 & \multicolumn{3}{|c|}{ Lab ID: $\quad 1009008-07$} & & & \\
\hline $14092-98-9$ & Chromium & $<1.72 \mathrm{E}-2$ & ug/g dry & $1.72 \mathrm{E}-2$ & $1 / 17 / 11$ & $1 \mathrm{~A} 17003$ & PNNL-AGG-415 \\
\hline $7440-38-2$ & Arsenic & $<7.09 \mathrm{E}-2$ & ug/g dry & $7.09 \mathrm{E}-2$ & $1 / 21 / 11$ & 1A17003 & PNNL-AGG-415 \\
\hline $14687-58-2$ & Selenium & $<3.96 \mathrm{E}-2$ & ug/g dry & $3.96 \mathrm{E}-2$ & $1 / 17 / 11$ & $1 \mathrm{~A} 17003$ & PNNL-AGG-415 \\
\hline $14378-37-1$ & Silver & $<1.56 \mathrm{E}-2$ & ug/g dry & $1.56 \mathrm{E}-2$ & $1 / 17 / 11$ & $1 \mathrm{~A} 17003$ & PNNL-AGG-415 \\
\hline $14336-64-2$ & Cadmium & $<2.86 \mathrm{E}-3$ & ug/g dry & $2.86 \mathrm{E}-3$ & $1 / 17 / 11$ & $1 \mathrm{~A} 17003$ & PNNL-AGG-415 \\
\hline $13966-28-4$ & Lead & $<5.81 \mathrm{E}-3$ & ug/g dry & $5.81 \mathrm{E}-3$ & $1 / 17 / 11$ & $1 \mathrm{~A} 17003$ & PNNL-AGG-415 \\
\hline
\end{tabular}


RCRA Metals By PNNL-AGG-415/1:5 Water Extract

\begin{tabular}{|c|c|c|c|c|c|c|c|}
\hline CAS \# & Analyte & Results & Units & EQL & Analyzed & Batch & Method \\
\hline HEIS No. & B273B3 & \multicolumn{3}{|c|}{ Lab ID: $\quad 1009008-08$} & & & \\
\hline 14092-98-9 & Chromium & $<1.70 \mathrm{E}-2$ & ug/g dry & $1.70 \mathrm{E}-2$ & $1 / 17 / 11$ & $1 \mathrm{~A} 17003$ & PNNL-AGG-415 \\
\hline $7440-38-2$ & Arsenic & $<6.99 \mathrm{E}-2$ & ug/g dry & $6.99 \mathrm{E}-2$ & $1 / 21 / 11$ & $1 \mathrm{~A} 17003$ & PNNL-AGG-415 \\
\hline $14687-58-2$ & Selenium & $<3.90 \mathrm{E}-2$ & ug/g dry & $3.90 \mathrm{E}-2$ & $1 / 17 / 11$ & $1 \mathrm{~A} 17003$ & PNNL-AGG-415 \\
\hline $14378-37-1$ & Silver & $<1.54 \mathrm{E}-2$ & ug/g dry & $1.54 \mathrm{E}-2$ & $1 / 17 / 11$ & $1 \mathrm{~A} 17003$ & PNNL-AGG-415 \\
\hline $14336-64-2$ & Cadmium & $<2.82 \mathrm{E}-3$ & ug/g dry & $2.82 \mathrm{E}-3$ & $1 / 17 / 11$ & $1 \mathrm{~A} 17003$ & PNNL-AGG-415 \\
\hline $13966-28-4$ & Lead & $<5.73 \mathrm{E}-3$ & ug/g dry & $5.73 \mathrm{E}-3$ & $1 / 17 / 11$ & $1 \mathrm{~A} 17003$ & PNNL-AGG-415 \\
\hline HEIS No. & B273B4 & \multicolumn{3}{|c|}{ Lab ID: $\quad 1009008-09$} & & & \\
\hline $14092-98-9$ & Chromium & $<1.73 \mathrm{E}-2$ & ug/g dry & $1.73 \mathrm{E}-2$ & $1 / 17 / 11$ & $1 \mathrm{~A} 17003$ & PNNL-AGG-415 \\
\hline $7440-38-2$ & Arsenic & $<7.12 \mathrm{E}-2$ & ug/g dry & $7.12 \mathrm{E}-2$ & $1 / 21 / 11$ & $1 \mathrm{~A} 17003$ & PNNL-AGG-415 \\
\hline $14687-58-2$ & Selenium & $<3.97 \mathrm{E}-2$ & ug/g dry & $3.97 \mathrm{E}-2$ & $1 / 17 / 11$ & $1 \mathrm{~A} 17003$ & PNNL-AGG-415 \\
\hline $14378-37-1$ & Silver & $<1.57 \mathrm{E}-2$ & ug/g dry & $1.57 \mathrm{E}-2$ & $1 / 17 / 11$ & $1 \mathrm{~A} 17003$ & PNNL-AGG-415 \\
\hline $14336-64-2$ & Cadmium & $<2.88 \mathrm{E}-3$ & ug/g dry & $2.88 \mathrm{E}-3$ & $1 / 17 / 11$ & $1 \mathrm{~A} 17003$ & PNNL-AGG-415 \\
\hline $13966-28-4$ & Lead & $5.95 \mathrm{E}-2$ & ug/g dry & $5.83 \mathrm{E}-3$ & $1 / 17 / 11$ & $1 \mathrm{~A} 17003$ & PNNL-AGG-415 \\
\hline HEIS No. & B273B6 & \multicolumn{3}{|c|}{ Lab ID: $\quad 1009008-10$} & & & \\
\hline $14092-98-9$ & Chromium & $2.20 \mathrm{E}-2$ & ug/g dry & $1.71 \mathrm{E}-2$ & $1 / 17 / 11$ & $1 \mathrm{~A} 17003$ & PNNL-AGG-415 \\
\hline $7440-38-2$ & Arsenic & $1.65 \mathrm{E}-1$ & ug/g dry & 7.06E-2 & $1 / 21 / 11$ & $1 \mathrm{~A} 17003$ & PNNL-AGG-415 \\
\hline $14687-58-2$ & Selenium & $<3.94 \mathrm{E}-2$ & ug/g dry & $3.94 \mathrm{E}-2$ & $1 / 17 / 11$ & $1 \mathrm{~A} 17003$ & PNNL-AGG-415 \\
\hline $14378-37-1$ & Silver & $<1.56 \mathrm{E}-2$ & ug/g dry & $1.56 \mathrm{E}-2$ & $1 / 17 / 11$ & $1 \mathrm{~A} 17003$ & PNNL-AGG-415 \\
\hline $14336-64-2$ & Cadmium & $<2.85 \mathrm{E}-3$ & ug/g dry & $2.85 \mathrm{E}-3$ & $1 / 17 / 11$ & $1 \mathrm{~A} 17003$ & PNNL-AGG-415 \\
\hline $13966-28-4$ & Lead & $<5.79 \mathrm{E}-3$ & ug/g dry & $5.79 \mathrm{E}-3$ & $1 / 17 / 11$ & $1 \mathrm{~A} 17003$ & PNNL-AGG-415 \\
\hline HEIS No. & B273B5 & \multicolumn{3}{|c|}{ Lab ID: $\quad 1009008-11$} & & & \\
\hline $14092-98-9$ & Chromium & $<1.68 \mathrm{E}-2$ & ug/g dry & $1.68 \mathrm{E}-2$ & $1 / 17 / 11$ & $1 \mathrm{~A} 17003$ & PNNL-AGG-415 \\
\hline $7440-38-2$ & Arsenic & $<6.93 \mathrm{E}-2$ & ug/g dry & $6.93 \mathrm{E}-2$ & $1 / 21 / 11$ & $1 \mathrm{~A} 17003$ & PNNL-AGG-415 \\
\hline $14687-58-2$ & Selenium & $<3.87 \mathrm{E}-2$ & ug/g dry & $3.87 \mathrm{E}-2$ & $1 / 17 / 11$ & $1 \mathrm{~A} 17003$ & PNNL-AGG-415 \\
\hline $14378-37-1$ & Silver & $<1.53 \mathrm{E}-2$ & ug/g dry & $1.53 \mathrm{E}-2$ & $1 / 17 / 11$ & 1A17003 & PNNL-AGG-415 \\
\hline $14336-64-2$ & Cadmium & $<2.80 \mathrm{E}-3$ & ug/g dry & $2.80 \mathrm{E}-3$ & $1 / 17 / 11$ & 1A17003 & PNNL-AGG-415 \\
\hline $13966-28-4$ & Lead & $<5.68 \mathrm{E}-3$ & ug/g dry & $5.68 \mathrm{E}-3$ & $1 / 17 / 11$ & $1 \mathrm{~A} 17003$ & PNNL-AGG-415 \\
\hline
\end{tabular}


RCRA Metals By PNNL-AGG-415/Acid Extract

\begin{tabular}{|c|c|c|c|c|c|c|c|}
\hline CAS \# & Analyte & Results & Units & EQL & Analyzed & Batch & Method \\
\hline HEIS No. & B274K7 & \multicolumn{3}{|c|}{ Lab ID: $\quad$ 1009008-01 } & & & \\
\hline 14092-98-9 & Chromium & $4.52 \mathrm{E} 0$ & ug/g dry & $4.36 \mathrm{E}-1$ & $10 / 11 / 10$ & 0J11001 & PNNL-AGG-415 \\
\hline $7440-38-2$ & Arsenic & $4.56 \mathrm{E}-1$ & ug/g dry & $1.90 \mathrm{E}-1$ & $10 / 11 / 10$ & $0 \mathrm{~J} 11001$ & PNNL-AGG-415 \\
\hline $14687-58-2$ & Selenium & $<3.67 \mathrm{E}-1$ & ug/g dry & $3.67 \mathrm{E}-1$ & $10 / 11 / 10$ & 0J11001 & PNNL-AGG-415 \\
\hline $14378-37-1$ & Silver & $6.20 \mathrm{E}-2$ & ug/g dry & $1.19 \mathrm{E}-2$ & $12 / 02 / 10$ & 0J11001 & PNNL-AGG-415 \\
\hline $14336-64-2$ & Cadmium & $<3.13 \mathrm{E}-2$ & ug/g dry & $3.13 \mathrm{E}-2$ & $12 / 02 / 10$ & 0J11001 & PNNL-AGG-415 \\
\hline 13966-28-4 & Lead & $2.83 \mathrm{E} 0$ & ug/g dry & $1.19 \mathrm{E}-1$ & $12 / 02 / 10$ & $0 \mathrm{~J} 11001$ & PNNL-AGG-415 \\
\hline HEIS No. & B273B8 & \multicolumn{3}{|c|}{ Lab ID: $\quad 1009008-02$} & & & \\
\hline 14092-98-9 & Chromium & $2.70 \mathrm{E} 0$ & ug/g dry & $3.91 \mathrm{E}-1$ & $10 / 11 / 10$ & $0 \mathrm{~J} 11001$ & PNNL-AGG-415 \\
\hline $7440-38-2$ & Arsenic & $3.92 \mathrm{E}-1$ & ug/g dry & $1.70 \mathrm{E}-1$ & $10 / 11 / 10$ & 0J11001 & PNNL-AGG-415 \\
\hline $14687-58-2$ & Selenium & $<3.29 \mathrm{E}-1$ & ug/g dry & $3.29 \mathrm{E}-1$ & $10 / 11 / 10$ & 0J11001 & PNNL-AGG-415 \\
\hline $14378-37-1$ & Silver & $2.98 \mathrm{E}-2$ & ug/g dry & $1.06 \mathrm{E}-2$ & $12 / 02 / 10$ & 0J11001 & PNNL-AGG-415 \\
\hline $14336-64-2$ & Cadmium & $<2.81 \mathrm{E}-2$ & ug/g dry & $2.81 \mathrm{E}-2$ & $12 / 02 / 10$ & 0J11001 & PNNL-AGG-415 \\
\hline $13966-28-4$ & Lead & $1.36 \mathrm{E} 0$ & ug/g dry & $1.06 \mathrm{E}-1$ & $12 / 02 / 10$ & 0J11001 & PNNL-AGG-415 \\
\hline HEIS No. & B27PY0 & \multicolumn{3}{|c|}{ Lab ID: $\quad 1009008-03$} & & & \\
\hline 14092-98-9 & Chromium & $2.53 \mathrm{E} 0$ & ug/g dry & $3.64 \mathrm{E}-1$ & $10 / 11 / 10$ & 0J11001 & PNNL-AGG-415 \\
\hline $7440-38-2$ & Arsenic & $3.08 \mathrm{E}-1$ & ug/g dry & $1.58 \mathrm{E}-1$ & $10 / 11 / 10$ & 0J11001 & PNNL-AGG-415 \\
\hline $14687-58-2$ & Selenium & $<3.06 \mathrm{E}-1$ & ug/g dry & $3.06 \mathrm{E}-1$ & $10 / 11 / 10$ & 0J11001 & PNNL-AGG-415 \\
\hline $14378-37-1$ & Silver & $2.65 \mathrm{E}-2$ & ug/g dry & $9.92 \mathrm{E}-3$ & $12 / 02 / 10$ & 0J11001 & PNNL-AGG-415 \\
\hline $14336-64-2$ & Cadmium & $3.08 \mathrm{E}-2$ & ug/g dry & $2.62 \mathrm{E}-2$ & $12 / 02 / 10$ & 0J11001 & PNNL-AGG-415 \\
\hline 13966-28-4 & Lead & $1.13 \mathrm{E} 0$ & ug/g dry & $9.92 \mathrm{E}-2$ & $12 / 02 / 10$ & $0 \mathrm{~J} 11001$ & PNNL-AGG-415 \\
\hline HEIS No. & В274K6 & \multicolumn{3}{|c|}{ Lab ID: $\quad 1009008-04$} & & & \\
\hline 14092-98-9 & Chromium & $2.71 \mathrm{E} 0$ & ug/g dry & $3.92 \mathrm{E}-1$ & $10 / 11 / 10$ & 0J11001 & PNNL-AGG-415 \\
\hline $7440-38-2$ & Arsenic & $3.32 \mathrm{E}-1$ & ug/g dry & $1.71 \mathrm{E}-1$ & $10 / 11 / 10$ & 0J11001 & PNNL-AGG-415 \\
\hline $14687-58-2$ & Selenium & $<3.30 \mathrm{E}-1$ & ug/g dry & $3.30 \mathrm{E}-1$ & $10 / 11 / 10$ & 0J11001 & PNNL-AGG-415 \\
\hline $14378-37-1$ & Silver & $2.40 \mathrm{E}-2$ & ug/g dry & $1.07 \mathrm{E}-2$ & $12 / 02 / 10$ & 0J11001 & PNNL-AGG-415 \\
\hline $14336-64-2$ & Cadmium & $<2.82 \mathrm{E}-2$ & ug/g dry & $2.82 \mathrm{E}-2$ & $12 / 02 / 10$ & 0J11001 & PNNL-AGG-415 \\
\hline 13966-28-4 & Lead & $1.24 \mathrm{E} 0$ & ug/g dry & $1.07 \mathrm{E}-1$ & $12 / 02 / 10$ & 0J11001 & PNNL-AGG-415 \\
\hline HEIS No. & B26TC1 & \multicolumn{3}{|c|}{ Lab ID: $\quad$ 1009008-05 } & & & \\
\hline 14092-98-9 & Chromium & $3.44 \mathrm{E} 0$ & ug/g dry & $3.54 \mathrm{E}-1$ & $10 / 11 / 10$ & $0 \mathrm{~J} 11001$ & PNNL-AGG-415 \\
\hline $7440-38-2$ & Arsenic & $2.69 \mathrm{E}-1$ & ug/g dry & $1.54 \mathrm{E}-1$ & $10 / 11 / 10$ & 0J11001 & PNNL-AGG-415 \\
\hline $14687-58-2$ & Selenium & $<2.98 \mathrm{E}-1$ & ug/g dry & $2.98 \mathrm{E}-1$ & $10 / 11 / 10$ & 0J11001 & PNNL-AGG-415 \\
\hline $14378-37-1$ & Silver & $3.46 \mathrm{E}-2$ & ug/g dry & $9.64 \mathrm{E}-3$ & $12 / 02 / 10$ & 0J11001 & PNNL-AGG-415 \\
\hline $14336-64-2$ & Cadmium & $3.43 \mathrm{E}-2$ & ug/g dry & $2.54 \mathrm{E}-2$ & $12 / 02 / 10$ & 0J11001 & PNNL-AGG-415 \\
\hline 13966-28-4 & Lead & $1.13 \mathrm{E} 0$ & ug/g dry & $9.65 \mathrm{E}-2$ & $12 / 02 / 10$ & 0J11001 & PNNL-AGG-415 \\
\hline HEIS No. & B26TC2 & \multicolumn{3}{|c|}{ Lab ID: $\quad 1009008-06$} & & & \\
\hline $14092-98-9$ & Chromium & $5.89 \mathrm{E} 0$ & ug/g dry & $3.93 \mathrm{E}-1$ & $10 / 11 / 10$ & 0J11001 & PNNL-AGG-415 \\
\hline $7440-38-2$ & Arsenic & $8.05 \mathrm{E}-1$ & ug/g dry & $1.71 \mathrm{E}-1$ & $10 / 11 / 10$ & 0J11001 & PNNL-AGG-415 \\
\hline $14687-58-2$ & Selenium & $<3.31 \mathrm{E}-1$ & ug/g dry & $3.31 \mathrm{E}-1$ & $10 / 11 / 10$ & 0J11001 & PNNL-AGG-415 \\
\hline $14378-37-1$ & Silver & $1.04 \mathrm{E}-1$ & ug/g dry & $1.07 \mathrm{E}-2$ & $12 / 02 / 10$ & 0J11001 & PNNL-AGG-415 \\
\hline $14336-64-2$ & Cadmium & $2.53 \mathrm{E}-1$ & ug/g dry & $2.83 \mathrm{E}-2$ & $12 / 02 / 10$ & 0J11001 & PNNL-AGG-415 \\
\hline 13966-28-4 & Lead & 3.67E0 & ug/g dry & $1.07 \mathrm{E}-1$ & $12 / 02 / 10$ & $0 \mathrm{~J} 11001$ & PNNL-AGG-415 \\
\hline HEIS No. & B273B2 & \multicolumn{3}{|c|}{ Lab ID: $\quad$ 1009008-07 } & & & \\
\hline 14092-98-9 & Chromium & $1.06 \mathrm{E} 1$ & ug/g dry & $6.80 \mathrm{E}-1$ & $1 / 20 / 11$ & $1 \mathrm{~A} 20001$ & PNNL-AGG-415 \\
\hline $7440-38-2$ & Arsenic & $2.62 \mathrm{E} 0$ & ug/g dry & $2.96 \mathrm{E}-1$ & $1 / 21 / 11$ & 1A20001 & PNNL-AGG-415 \\
\hline $14687-58-2$ & Selenium & $<5.72 \mathrm{E}-1$ & ug/g dry & $5.72 \mathrm{E}-1$ & $1 / 20 / 11$ & 1A20001 & PNNL-AGG-415 \\
\hline $14378-37-1$ & Silver & $7.18 \mathrm{E}-2$ & ug/g dry & $1.85 \mathrm{E}-2$ & $1 / 20 / 11$ & 1A20001 & PNNL-AGG-415 \\
\hline 14336-64-2 & Cadmium & $3.04 \mathrm{E}-1$ & ug/g dry & $4.89 \mathrm{E}-2$ & $1 / 20 / 11$ & 1A20001 & PNNL-AGG-415 \\
\hline 13966-28-4 & Lead & $3.95 \mathrm{E} 0$ & ug/g dry & $1.85 \mathrm{E}-1$ & $1 / 20 / 11$ & 1A20001 & PNNL-AGG-415 \\
\hline
\end{tabular}


RCRA Metals By PNNL-AGG-415/Acid Extract

\begin{tabular}{|c|c|c|c|c|c|c|c|}
\hline CAS \# & Analyte & Results & Units & EQL & Analyzed & Batch & Method \\
\hline HEIS No. & B273B3 & \multicolumn{3}{|c|}{ Lab ID: $\quad 1009008-08$} & & & \\
\hline $14092-98-9$ & Chromium & $1.07 \mathrm{E} 1$ & ug/g dry & $7.76 \mathrm{E}-1$ & $1 / 20 / 11$ & 1A20001 & PNNL-AGG-415 \\
\hline $7440-38-2$ & Arsenic & $2.54 \mathrm{E} 0$ & ug/g dry & $3.38 \mathrm{E}-1$ & $1 / 21 / 11$ & 1A20001 & PNNL-AGG-415 \\
\hline $14687-58-2$ & Selenium & $<6.53 \mathrm{E}-1$ & ug/g dry & $6.53 \mathrm{E}-1$ & $1 / 20 / 11$ & $1 \mathrm{~A} 20001$ & PNNL-AGG-415 \\
\hline $14378-37-1$ & Silver & $5.49 \mathrm{E}-2$ & ug/g dry & $2.11 \mathrm{E}-2$ & $1 / 20 / 11$ & 1A20001 & PNNL-AGG-415 \\
\hline $14336-64-2$ & Cadmium & $1.34 \mathrm{E}-1$ & ug/g dry & $5.58 \mathrm{E}-2$ & $1 / 20 / 11$ & 1A20001 & PNNL-AGG-415 \\
\hline $13966-28-4$ & Lead & 2.59E0 & ug/g dry & $2.12 \mathrm{E}-1$ & $1 / 20 / 11$ & $1 \mathrm{~A} 20001$ & PNNL-AGG-415 \\
\hline HEIS No. & B273B4 & \multicolumn{3}{|c|}{ Lab ID: $\quad 1009008-09$} & & & \\
\hline 14092-98-9 & Chromium & $1.49 \mathrm{E} 1$ & ug/g dry & $7.32 \mathrm{E}-1$ & $1 / 20 / 11$ & 1A20001 & PNNL-AGG-415 \\
\hline $7440-38-2$ & Arsenic & $3.06 \mathrm{E} 0$ & ug/g dry & $3.19 \mathrm{E}-1$ & $1 / 22 / 11$ & $1 \mathrm{~A} 20001$ & PNNL-AGG-415 \\
\hline $14687-58-2$ & Selenium & $<6.16 \mathrm{E}-1$ & ug/g dry & $6.16 \mathrm{E}-1$ & $1 / 20 / 11$ & $1 \mathrm{~A} 20001$ & PNNL-AGG-415 \\
\hline $14378-37-1$ & Silver & $7.76 \mathrm{E}-2$ & ug/g dry & $1.99 \mathrm{E}-2$ & $1 / 20 / 11$ & 1A20001 & PNNL-AGG-415 \\
\hline $14336-64-2$ & Cadmium & $1.92 \mathrm{E}-1$ & ug/g dry & $5.26 \mathrm{E}-2$ & $1 / 20 / 11$ & 1A20001 & PNNL-AGG-415 \\
\hline $13966-28-4$ & Lead & $4.27 \mathrm{E} 0$ & ug/g dry & $2.00 \mathrm{E}-1$ & $1 / 20 / 11$ & 1A20001 & PNNL-AGG-415 \\
\hline HEIS No. & B273B6 & \multicolumn{3}{|c|}{ Lab ID: $\quad 1009008-10$} & & & \\
\hline $14092-98-9$ & Chromium & $1.10 \mathrm{E} 1$ & ug/g dry & $8.95 \mathrm{E}-1$ & $1 / 20 / 11$ & $1 \mathrm{~A} 20001$ & PNNL-AGG-415 \\
\hline $7440-38-2$ & Arsenic & $9.71 \mathrm{E} 0$ & ug/g dry & $3.90 \mathrm{E}-1$ & $1 / 22 / 11$ & 1A20001 & PNNL-AGG-415 \\
\hline $14687-58-2$ & Selenium & $<7.53 \mathrm{E}-1$ & ug/g dry & $7.53 \mathrm{E}-1$ & $1 / 20 / 11$ & 1A20001 & PNNL-AGG-415 \\
\hline $14378-37-1$ & Silver & $<2.44 \mathrm{E}-2$ & ug/g dry & $2.44 \mathrm{E}-2$ & $1 / 20 / 11$ & 1A20001 & PNNL-AGG-415 \\
\hline $14336-64-2$ & Cadmium & $<6.43 \mathrm{E}-2$ & ug/g dry & $6.43 \mathrm{E}-2$ & $1 / 20 / 11$ & 1A20001 & PNNL-AGG-415 \\
\hline $13966-28-4$ & Lead & $5.10 \mathrm{E} 0$ & ug/g dry & $2.44 \mathrm{E}-1$ & $1 / 20 / 11$ & 1A20001 & PNNL-AGG-415 \\
\hline HEIS No. & B273B5 & \multicolumn{3}{|c|}{ Lab ID: $\quad 1009008-11$} & & & \\
\hline $14092-98-9$ & Chromium & $9.88 \mathrm{E} 0$ & ug/g dry & 8.44E-1 & $1 / 20 / 11$ & 1A20001 & PNNL-AGG-415 \\
\hline $7440-38-2$ & Arsenic & $2.16 \mathrm{E} 0$ & ug/g dry & $3.67 \mathrm{E}-1$ & $1 / 22 / 11$ & 1A20001 & PNNL-AGG-415 \\
\hline $14687-58-2$ & Selenium & $<7.10 \mathrm{E}-1$ & ug/g dry & 7.10E-1 & $1 / 20 / 11$ & 1A20001 & PNNL-AGG-415 \\
\hline $14378-37-1$ & Silver & $5.08 \mathrm{E}-2$ & ug/g dry & $2.30 \mathrm{E}-2$ & $1 / 20 / 11$ & 1A20001 & PNNL-AGG-415 \\
\hline $14336-64-2$ & Cadmium & $6.22 \mathrm{E}-2$ & ug/g dry & $6.07 \mathrm{E}-2$ & $1 / 20 / 11$ & $1 \mathrm{~A} 20001$ & PNNL-AGG-415 \\
\hline $13966-28-4$ & Lead & 4.03E0 & ug/g dry & $2.30 \mathrm{E}-1$ & $1 / 20 / 11$ & 1A20001 & PNNL-AGG-415 \\
\hline
\end{tabular}


Strontium 90/Acid Extract

Strontium-90 (pCi/g dry) by AGG-RRL-002

\begin{tabular}{|c|c|c|c|c|c|c|}
\hline Lab ID & HEIS No. & Results & MDA & UNC & Analyzed & Batch \\
\hline $1009008-01$ & B274K7 & $<9.11 \mathrm{E}-1$ & $9.11 \mathrm{E}-1$ & & $10 / 15 / 10$ & 0J07001 \\
\hline $1009008-02$ & B273B8 & $<9.04 \mathrm{E}-1$ & $9.04 \mathrm{E}-1$ & & $10 / 15 / 10$ & 0J07001 \\
\hline $1009008-03$ & B27PY0 & $<9.02 \mathrm{E}-1$ & $9.02 \mathrm{E}-1$ & & $10 / 15 / 10$ & 0J07001 \\
\hline $1009008-04$ & B274K6 & $<8.96 \mathrm{E}-1$ & $8.96 \mathrm{E}-1$ & & $10 / 15 / 10$ & 0J07001 \\
\hline $1009008-05$ & B26TC1 & $<8.99 \mathrm{E}-1$ & $8.99 \mathrm{E}-1$ & & $10 / 15 / 10$ & 0J07001 \\
\hline 1009008-06 & B26TC2 & $<8.89 \mathrm{E}-1$ & $8.89 \mathrm{E}-1$ & & $10 / 15 / 10$ & 0J07001 \\
\hline $1009008-07$ & B273B2 & $<1.11 \mathrm{E} 0$ & $1.11 \mathrm{E} 0$ & & $1 / 12 / 11$ & $1 \mathrm{~A} 05001$ \\
\hline 1009008-08 & B273B3 & $<1.10 \mathrm{E} 0$ & $1.10 \mathrm{E} 0$ & & $1 / 12 / 11$ & $1 \mathrm{~A} 05001$ \\
\hline 1009008-09 & B273B4 & $<1.12 \mathrm{E} 0$ & $1.12 \mathrm{E} 0$ & & $1 / 12 / 11$ & $1 \mathrm{~A} 05001$ \\
\hline $1009008-10$ & B273B6 & $<1.10 \mathrm{E} 0$ & $1.10 \mathrm{E} 0$ & & $1 / 12 / 11$ & $1 \mathrm{~A} 05001$ \\
\hline $1009008-11$ & B273B5 & $<1.10 \mathrm{E} 0$ & $1.10 \mathrm{E} 0$ & & $1 / 12 / 11$ & $1 \mathrm{~A} 05001$ \\
\hline
\end{tabular}


Strontium 90/1:1 Water Extract

Strontium-90 (pCi/g dry) by AGG-RRL-002

\begin{tabular}{|c|c|c|c|c|c|c|}
\hline Lab ID & HEIS No. & Results & MDA & UNC & Analyzed & Batch \\
\hline $1009008-01$ & B274K7 & $<8.74 \mathrm{E}-1$ & $8.74 \mathrm{E}-1$ & & $10 / 15 / 10$ & 0J07010 \\
\hline $1009008-02$ & B273B8 & $<9.29 \mathrm{E}-1$ & $9.29 \mathrm{E}-1$ & & $10 / 15 / 10$ & 0J07010 \\
\hline $1009008-03$ & B27PY0 & $<9.43 \mathrm{E}-1$ & $9.43 \mathrm{E}-1$ & & $10 / 15 / 10$ & 0J07010 \\
\hline $1009008-04$ & B274K6 & $<9.34 \mathrm{E}-1$ & $9.34 \mathrm{E}-1$ & & $10 / 15 / 10$ & 0J07010 \\
\hline $1009008-05$ & B26TC1 & $<9.32 \mathrm{E}-1$ & $9.32 \mathrm{E}-1$ & & $10 / 15 / 10$ & 0J07010 \\
\hline 1009008-06 & B26TC2 & $<9.70 \mathrm{E}-1$ & $9.70 \mathrm{E}-1$ & & $10 / 15 / 10$ & 0J07010 \\
\hline 1009008-07 & B273B2 & $<1.12 \mathrm{E} 0$ & $1.12 \mathrm{E} 0$ & & $1 / 12 / 11$ & $1 \mathrm{~A} 06003$ \\
\hline 1009008-08 & B273B3 & $<1.12 \mathrm{E} 0$ & $1.12 \mathrm{E} 0$ & & $1 / 12 / 11$ & $1 \mathrm{~A} 06003$ \\
\hline 1009008-09 & B273B4 & $<1.12 \mathrm{E} 0$ & $1.12 \mathrm{E} 0$ & & $1 / 12 / 11$ & $1 \mathrm{~A} 06003$ \\
\hline $1009008-10$ & B273B6 & $<1.11 \mathrm{E} 0$ & $1.11 \mathrm{E} 0$ & & $1 / 12 / 11$ & $1 \mathrm{~A} 06003$ \\
\hline $1009008-11$ & B273B5 & $<1.16 \mathrm{E} 0$ & $1.16 \mathrm{E} 0$ & & $1 / 12 / 11$ & $1 \mathrm{~A} 06003$ \\
\hline
\end{tabular}




\section{Strontium 90/1:2.5 Water Extract}

Strontium-90 (pCi/g dry) by AGG-RRL-002

\begin{tabular}{|c|c|c|c|c|c|}
\hline Lab ID & HEIS No. & Results & MDA & Analyzed & Batch \\
\hline $1009008-01$ & B274K7 & $<2.22 \mathrm{E} 0$ & $2.22 \mathrm{E} 0$ & $11 / 15 / 10$ & 0J07011 \\
\hline $1009008-02$ & B273B8 & $<2.32 \mathrm{E} 0$ & $2.32 \mathrm{E} 0$ & $11 / 15 / 10$ & 0J07011 \\
\hline $1009008-03$ & B27PY0 & $<2.33 \mathrm{E} 0$ & $2.33 \mathrm{E} 0$ & $11 / 15 / 10$ & 0J07011 \\
\hline 1009008-04 & B274K6 & $<2.48 \mathrm{E} 0$ & $2.48 \mathrm{E} 0$ & $11 / 15 / 10$ & 0J07011 \\
\hline $1009008-05$ & B26TC1 & $<2.32 \mathrm{E} 0$ & $2.32 \mathrm{E} 0$ & $11 / 15 / 10$ & 0J07011 \\
\hline 1009008-06 & B26TC2 & $<2.31 \mathrm{E} 0$ & $2.31 \mathrm{E} 0$ & $11 / 15 / 10$ & 0J07011 \\
\hline $1009008-07$ & B273B2 & $<2.75 \mathrm{E} 0$ & $2.75 \mathrm{E} 0$ & $1 / 17 / 11$ & $1 \mathrm{~A} 07003$ \\
\hline $1009008-08$ & B273B3 & $<2.75 \mathrm{E} 0$ & $2.75 \mathrm{E} 0$ & $1 / 17 / 11$ & $1 \mathrm{~A} 07003$ \\
\hline 1009008-09 & B273B4 & $<2.76 \mathrm{E} 0$ & $2.76 \mathrm{E} 0$ & $1 / 17 / 11$ & $1 \mathrm{~A} 07003$ \\
\hline $1009008-10$ & B273B6 & $<2.76 \mathrm{E} 0$ & $2.76 \mathrm{E} 0$ & $1 / 17 / 11$ & $1 \mathrm{~A} 07003$ \\
\hline $1009008-11$ & B273B5 & $<2.77 \mathrm{E} 0$ & $2.77 \mathrm{E} 0$ & $1 / 17 / 11$ & $1 \mathrm{~A} 07003$ \\
\hline
\end{tabular}


Strontium 90/1:5 Water Extract

Strontium-90 (pCi/g dry) by AGG-RRL-002

\begin{tabular}{|c|c|c|c|c|c|c|}
\hline Lab ID & HEIS No. & Results & MDA & UNC & Analyzed & Batch \\
\hline $1009008-01$ & B274K7 & $<4.47 \mathrm{E} 0$ & 4.47E0 & & $10 / 15 / 10$ & 0J07012 \\
\hline 1009008-02 & B273B8 & $<4.61 \mathrm{E} 0$ & 4.61E0 & & $10 / 15 / 10$ & 0J07012 \\
\hline $1009008-03$ & B27PY0 & $<4.28 \mathrm{E} 0$ & $4.28 \mathrm{E} 0$ & & $10 / 15 / 10$ & 0J07012 \\
\hline $1009008-04$ & B274K6 & $<4.65 \mathrm{E} 0$ & $4.65 \mathrm{E} 0$ & & $10 / 15 / 10$ & 0J07012 \\
\hline $1009008-05$ & B26TC1 & $<4.58 \mathrm{E} 0$ & $4.58 \mathrm{E} 0$ & & $10 / 15 / 10$ & 0J07012 \\
\hline 1009008-06 & B26TC2 & $<4.64 \mathrm{E} 0$ & 4.64E0 & & $10 / 15 / 10$ & 0J07012 \\
\hline 1009008-07 & B273B2 & $<5.54 \mathrm{E} 0$ & $5.54 \mathrm{E} 0$ & & $1 / 17 / 11$ & 1A10006 \\
\hline 1009008-08 & B273B3 & $<5.46 \mathrm{E} 0$ & $5.46 \mathrm{E} 0$ & & $1 / 17 / 11$ & $1 \mathrm{~A} 10006$ \\
\hline 1009008-09 & B273B4 & $<5.57 \mathrm{E} 0$ & $5.57 \mathrm{E} 0$ & & $1 / 17 / 11$ & $1 \mathrm{~A} 10006$ \\
\hline $1009008-10$ & B273B6 & $<5.52 \mathrm{E} 0$ & $5.52 \mathrm{E} 0$ & & $1 / 17 / 11$ & $1 \mathrm{~A} 10006$ \\
\hline $1009008-11$ & B273B5 & $<5.42 \mathrm{E} 0$ & $5.42 \mathrm{E} 0$ & & $1 / 17 / 11$ & $1 \mathrm{~A} 10006$ \\
\hline
\end{tabular}


Equilibrium Kd 1:1 Calculations

\begin{tabular}{|c|c|}
\hline $1009008-01$ & B274K7 \\
\hline Analyte & $\mathrm{Kd}(\mathrm{L} / \mathrm{g})$ \\
\hline Arsenic & $4.83 \mathrm{E}-02$ \\
\hline Barium & $\overline{N D}$ \\
\hline Cadmium & $\overline{N D}$ \\
\hline Chromium (Total) & ND \\
\hline Chromium, Hexavalent & ND \\
\hline Lead & $\overline{N D}$ \\
\hline Selenium & ND \\
\hline Silver & ND \\
\hline Strontium-90 & ND \\
\hline
\end{tabular}

1009008-02 B273B8

\begin{tabular}{|l|c|}
\hline Analyte & $\mathrm{Kd}(\mathrm{L} / \mathrm{g})$ \\
\hline Arsenic & $\mathrm{ND}$ \\
\hline Barium & $\mathrm{ND}$ \\
\hline Cadmium & $\mathrm{ND}$ \\
\hline Chromium (Total) & $\mathrm{ND}$ \\
\hline Chromium, Hexavalent & $\mathrm{ND}$ \\
\hline Lead & $\mathrm{ND}$ \\
\hline Selenium & $\mathrm{ND}$ \\
\hline Silver & $\mathrm{ND}$ \\
\hline Strontium-90 & $\mathrm{ND}$ \\
\hline
\end{tabular}

1009008-03
\begin{tabular}{|l|c|}
\hline Analyte & Kd (L/g) \\
\hline Arsenic & ND \\
\hline Barium & ND \\
\hline Cadmium & ND \\
\hline Chromium (Total) & ND \\
\hline Chromium, Hexavalent & ND \\
\hline Lead & ND \\
\hline Selenium & ND \\
\hline Silver & ND \\
\hline Strontium-90 & ND \\
\hline
\end{tabular}

1009008-04
\begin{tabular}{|l|c|}
\hline Analyte & K $274 K 6$ \\
\hline Arsenic & ND \\
\hline Barium & ND \\
\hline Cadmium & ND \\
\hline Chromium (Total) & ND \\
\hline Chromium, Hexavalent & ND \\
\hline Lead & ND \\
\hline Selenium & ND \\
\hline Silver & ND \\
\hline Strontium-90 & ND \\
\hline
\end{tabular}

1009008-05 B26TC1

1009008-06

B26TC2

\begin{tabular}{|l|c|}
\hline Analyte & $\mathrm{Kd}(\mathrm{L} / \mathrm{g})$ \\
\hline Arsenic & $\mathrm{ND}$ \\
\hline Barium & $\mathrm{ND}$ \\
\hline Cadmium & $\mathrm{ND}$ \\
\hline Chromium (Total) & $\mathrm{ND}$ \\
\hline Chromium, Hexavalent & $\mathrm{ND}$ \\
\hline Lead & $\mathrm{ND}$ \\
\hline Selenium & $\mathrm{ND}$ \\
\hline Silver & $\mathrm{ND}$ \\
\hline Strontium-90 & $\mathrm{ND}$ \\
\hline
\end{tabular}

\begin{tabular}{|l|c|}
\hline Analyte & Kd (L/g) \\
\hline Arsenic & $8.99 \mathrm{E}-02$ \\
\hline Barium & $\mathrm{ND}$ \\
\hline Cadmium & $\mathrm{ND}$ \\
\hline Chromium (Total) & $\mathrm{ND}$ \\
\hline Chromium, Hexavalent & $\mathrm{ND}$ \\
\hline Lead & $\mathrm{ND}$ \\
\hline Selenium & $\mathrm{ND}$ \\
\hline Silver & $\mathrm{ND}$ \\
\hline Strontium-90 & $\mathrm{ND}$ \\
\hline
\end{tabular}

\begin{tabular}{l} 
1009008-07 \\
\begin{tabular}{|l|c|}
\hline Analyte & B273B2 \\
\hline Arsenic & $\mathrm{Kd} \mathrm{(L/g)}$ \\
\hline Barium & $1.21 \mathrm{E}-01$ \\
\hline Cadmium & $\mathrm{ND}$ \\
\hline Chromium (Total) & $\mathrm{ND}$ \\
\hline Chromium, Hexavalent & $\mathrm{ND}$ \\
\hline Lead & $\mathrm{ND}$ \\
\hline Selenium & $\mathrm{ND}$ \\
\hline Silver & $\mathrm{ND}$ \\
\hline Strontium-90 & $\mathrm{ND}$ \\
\hline
\end{tabular} ND \\
\hline
\end{tabular}

$1009008-08$
\begin{tabular}{|l|c|}
\hline Analyte & Kd $(\mathrm{L} / \mathrm{g})$ \\
\hline Arsenic & $\mathrm{ND}$ \\
\hline Barium & $\mathrm{ND}$ \\
\hline Cadmium & $\mathrm{ND}$ \\
\hline Chromium (Total) & $\mathrm{ND}$ \\
\hline Chromium, Hexavalent & $\mathrm{ND}$ \\
\hline Lead & $\mathrm{ND}$ \\
\hline Selenium & $\mathrm{ND}$ \\
\hline Silver & $\mathrm{ND}$ \\
\hline Strontium-90 & $\mathrm{ND}$ \\
\hline
\end{tabular}

\begin{tabular}{|c|c|}
\hline 1009008-09 & B273B4 \\
\hline Analyte & $\overline{\mathrm{Kd}(\mathrm{L} / \mathrm{g})}$ \\
\hline Arsenic & $1.12 \mathrm{E}-01$ \\
\hline Barium & ND \\
\hline Cadmium & ND \\
\hline Chromium (Total) & ND \\
\hline Chromium, Hexavalent & ND \\
\hline Lead & ND \\
\hline Selenium & ND \\
\hline Silver & ND \\
\hline Strontium-90 & ND \\
\hline
\end{tabular}

1009008-10
\begin{tabular}{|l|c|}
\hline Analyte & K $273 \mathrm{~B} 6$ \\
\hline Arsenic & $2.44 \mathrm{E}-01$ \\
\hline Barium & $\mathrm{ND}$ \\
\hline Cadmium & $\mathrm{ND}$ \\
\hline Chromium (Total) & $5.11 \mathrm{E}-01$ \\
\hline Chromium, Hexavalent & $\mathrm{ND}$ \\
\hline Lead & $\mathrm{ND}$ \\
\hline Selenium & $\mathrm{ND}$ \\
\hline Silver & $\mathrm{ND}$ \\
\hline Strontium-90 & $\mathrm{ND}$ \\
\hline
\end{tabular}

1009008-11
\begin{tabular}{|l|c|}
\hline Analyte & Kd 273 (L/g) \\
\hline Arsenic & $1.13 \mathrm{E}-01$ \\
\hline Barium & $\mathrm{ND}$ \\
\hline Cadmium & $\mathrm{ND}$ \\
\hline Chromium (Total) & $\mathrm{ND}$ \\
\hline Chromium, Hexavalent & $\mathrm{ND}$ \\
\hline Lead & $\mathrm{ND}$ \\
\hline Selenium & $\mathrm{ND}$ \\
\hline Silver & $\mathrm{ND}$ \\
\hline Strontium-90 & $\mathrm{ND}$ \\
\hline
\end{tabular}




\begin{tabular}{|c|c|c|c|c|c|}
\hline \multicolumn{6}{|c|}{ Equilibrium Kd 1:2.5 Calculations } \\
\hline 1009008-01 & B274K7 & 1009008-02 & B273B8 & $1009008-03$ & B27PY0 \\
\hline Analyte & $\mathrm{Kd}(\mathrm{L} / \mathrm{g})$ & Analyte & $\mathrm{Kd}(\mathrm{L} / \mathrm{g})$ & Analyte & $\mathrm{Kd}(\mathrm{L} / \mathrm{g})$ \\
\hline Arsenic & $5.06 \mathrm{E}-02$ & Arsenic & ND & Arsenic & ND \\
\hline Barium & ND & Barium & ND & Barium & ND \\
\hline Cadmium & ND & Cadmium & ND & Cadmium & ND \\
\hline \begin{tabular}{|l} 
Chromium (Total) \\
\end{tabular} & ND & Chromium (Total) & ND & Chromium (Total) & ND \\
\hline Chromium, Hexavalent & ND & Chromium, Hexavalent & ND & Chromium, Hexavalent & ND \\
\hline Lead & ND & Lead & ND & Lead & ND \\
\hline $\begin{array}{l}\text { Selenium } \\
\end{array}$ & ND & Selenium & ND & Selenium & ND \\
\hline Silver & ND & Silver & ND & Silver & ND \\
\hline Strontium-90 & ND & Strontium-90 & ND & Strontium-90 & ND \\
\hline $1009008-04$ & B274K6 & $1009008-05$ & B26TC1 & $1009008-06$ & B26TC2 \\
\hline Analyte & $\mathrm{Kd}(\mathrm{L} / \mathrm{g})$ & Analyte & $\mathrm{Kd}(\mathrm{L} / \mathrm{g})$ & Analyte & $\mathrm{Kd}(\mathrm{L} / \mathrm{g})$ \\
\hline Arsenic & ND & Arsenic & ND & Arsenic & $6.19 \mathrm{E}-02$ \\
\hline Barium & ND & Barium & ND & Barium & ND \\
\hline Cadmium & ND & Cadmium & ND & Cadmium & ND \\
\hline Chromium (Total) & ND & Chromium (Total) & ND & Chromium (Total) & ND \\
\hline Chromium, Hexavalent & ND & Chromium, Hexavalent & ND & Chromium, Hexavalent & ND \\
\hline Lead & ND & Lead & ND & Lead & ND \\
\hline Selenium & ND & Selenium & ND & Selenium & ND \\
\hline Silver & ND & Silver & ND & Silver & ND \\
\hline Strontium-90 & ND & Strontium-90 & ND & Strontium-90 & ND \\
\hline 1009008-07 & B273B2 & $1009008-08$ & B273B3 & 1009008-09 & B273B4 \\
\hline Analyte & $\mathrm{Kd}(\mathrm{L} / \mathrm{g})$ & Analyte & $\mathrm{Kd}(\mathrm{L} / \mathrm{g})$ & Analyte & $\mathrm{Kd}(\mathrm{L} / \mathrm{g})$ \\
\hline Arsenic & $5.94 \mathrm{E}-02$ & Arsenic & $6.86 \mathrm{E}-02$ & Arsenic & $5.12 \mathrm{E}-02$ \\
\hline Barium & ND & Barium & ND & Barium & ND \\
\hline Cadmium & ND & Cadmium & ND & Cadmium & ND \\
\hline Chromium (Total) & ND & Chromium (Total) & ND & Chromium (Total) & ND \\
\hline Chromium, Hexavalent & ND & Chromium, Hexavalent & ND & Chromium, Hexavalent & ND \\
\hline Lead & ND & Lead & ND & Lead & ND \\
\hline Selenium & ND & Selenium & ND & Selenium & ND \\
\hline Silver & ND & Silver & ND & Silver & ND \\
\hline Strontium-90 & ND & Strontium-90 & ND & Strontium-90 & ND \\
\hline $1009008-10$ & B273B6 & 1009008-11 & B273B5 & & \\
\hline Analyte & $\mathrm{Kd}(\mathrm{L} / \mathrm{g})$ & Analyte & $\mathrm{Kd}(\mathrm{L} / \mathrm{g})$ & & \\
\hline Arsenic & $9.15 \mathrm{E}-02$ & Arsenic & ND & & \\
\hline Barium & ND & Barium & ND & & \\
\hline Cadmium & ND & Cadmium & ND & & \\
\hline Chromium (Total) & $5.15 \mathrm{E}-01$ & Chromium (Total) & ND & & \\
\hline Chromium, Hexavalent & ND & Chromium, Hexavalent & ND & & \\
\hline Lead & ND & Lead & ND & & \\
\hline Selenium & ND & Selenium & ND & & \\
\hline Silver & ND & Silver & ND & & \\
\hline Strontium-90 & ND & Strontium-90 & ND & & \\
\hline
\end{tabular}


Equilibrium Kd 1:5 Calculations

1009008-01
\begin{tabular}{|l|c|}
\hline Analyte & K274K7 \\
\hline Arsenic & $\mathrm{ND}$ \\
\hline Barium & $\mathrm{ND}$ \\
\hline Cadmium & $\mathrm{ND}$ \\
\hline Chromium (Total) & $\mathrm{ND}$ \\
\hline Chromium, Hexavalent & $\mathrm{ND}$ \\
\hline Lead & $\mathrm{ND}$ \\
\hline Selenium & $\mathrm{ND}$ \\
\hline Silver & $\mathrm{ND}$ \\
\hline Strontium-90 & $\mathrm{ND}$ \\
\hline
\end{tabular}

1009008-02 B273B8

\begin{tabular}{|l|c|}
\hline Analyte & $\mathrm{Kd}(\mathrm{L} / \mathrm{g})$ \\
\hline Arsenic & $\mathrm{ND}$ \\
\hline Barium & $\mathrm{ND}$ \\
\hline Cadmium & $\mathrm{ND}$ \\
\hline Chromium (Total) & $\mathrm{ND}$ \\
\hline Chromium, Hexavalent & $\mathrm{ND}$ \\
\hline Lead & $\mathrm{ND}$ \\
\hline Selenium & $\mathrm{ND}$ \\
\hline Silver & $\mathrm{ND}$ \\
\hline Strontium-90 & $\mathrm{ND}$ \\
\hline
\end{tabular}

1009008-03
\begin{tabular}{|l|c|}
\hline Analyte & Kd (L/g) \\
\hline Arsenic & ND \\
\hline Barium & ND \\
\hline Cadmium & ND \\
\hline Chromium (Total) & ND \\
\hline Chromium, Hexavalent & ND \\
\hline Lead & ND \\
\hline Selenium & ND \\
\hline Silver & ND \\
\hline Strontium-90 & ND \\
\hline
\end{tabular}

1009008-04
\begin{tabular}{|l|c|}
\hline Analyte & K274K6 \\
\hline Arsenic & ND \\
\hline Barium & ND \\
\hline Cadmium & ND \\
\hline Chromium (Total) & ND \\
\hline Chromium, Hexavalent & ND \\
\hline Lead & ND \\
\hline Selenium & ND \\
\hline Silver & ND \\
\hline Strontium-90 & ND \\
\hline
\end{tabular}

1009008-05 B26TC1

1009008-06

B26TC2

\begin{tabular}{|l|c|}
\hline Analyte & $\mathrm{Kd}(\mathrm{L} / \mathrm{g})$ \\
\hline Arsenic & $\mathrm{ND}$ \\
\hline Barium & $\mathrm{ND}$ \\
\hline Cadmium & $\mathrm{ND}$ \\
\hline Chromium (Total) & $\mathrm{ND}$ \\
\hline Chromium, Hexavalent & $\mathrm{ND}$ \\
\hline Lead & $\mathrm{ND}$ \\
\hline Selenium & $\mathrm{ND}$ \\
\hline Silver & $\mathrm{ND}$ \\
\hline Strontium-90 & $\mathrm{ND}$ \\
\hline
\end{tabular}

\begin{tabular}{|l|c|}
\hline Analyte & Kd (L/g) \\
\hline Arsenic & $3.93 \mathrm{E}-02$ \\
\hline Barium & ND \\
\hline Cadmium & ND \\
\hline Chromium (Total) & ND \\
\hline Chromium, Hexavalent & ND \\
\hline Lead & ND \\
\hline Selenium & ND \\
\hline Silver & ND \\
\hline Strontium-90 & ND \\
\hline
\end{tabular}

\begin{tabular}{|c|c|}
\hline 1009008-07 & B273B2 \\
\hline Analyte & $\mathrm{Kd}(\mathrm{L} / \mathrm{g})$ \\
\hline Arsenic & ND \\
\hline Barium & $\mathrm{ND}$ \\
\hline Cadmium & $\mathrm{ND}$ \\
\hline Chromium (Total) & ND \\
\hline Chromium, Hexavalent & ND \\
\hline Lead & ND \\
\hline Selenium & ND \\
\hline Silver & ND \\
\hline Strontium-90 & ND \\
\hline
\end{tabular}

$1009008-08$
\begin{tabular}{|l|c|}
\hline Analyte & Kd $(\mathrm{L} / \mathrm{g})$ \\
\hline Arsenic & $\mathrm{ND}$ \\
\hline Barium & $\mathrm{ND}$ \\
\hline Cadmium & $\mathrm{ND}$ \\
\hline Chromium (Total) & $\mathrm{ND}$ \\
\hline Chromium, Hexavalent & $\mathrm{ND}$ \\
\hline Lead & $\mathrm{ND}$ \\
\hline Selenium & $\mathrm{ND}$ \\
\hline Silver & $\mathrm{ND}$ \\
\hline Strontium-90 & $\mathrm{ND}$ \\
\hline
\end{tabular}

\begin{tabular}{|c|c|}
\hline 1009008-09 & B273B4 \\
\hline Analyte & $\mathrm{Kd}(\mathrm{L} / \mathrm{g})$ \\
\hline Arsenic & ND \\
\hline Barium & ND \\
\hline Cadmium & ND \\
\hline Chromium (Total) & ND \\
\hline Chromium, Hexavalent & ND \\
\hline Lead & 7.08E-02 \\
\hline Selenium & ND \\
\hline Silver & ND \\
\hline Strontium-90 & ND \\
\hline
\end{tabular}

1009008-10
\begin{tabular}{|l|c|}
\hline Analyte & B $273 \mathrm{Bd} 6$ \\
\hline Arsenic & $5.78 \mathrm{E}-02$ \\
\hline Barium & $\mathrm{ND}$ \\
\hline Cadmium & $\mathrm{ND}$ \\
\hline Chromium (Total) & $4.99 \mathrm{E}-01$ \\
\hline Chromium, Hexavalent & $\mathrm{ND}$ \\
\hline Lead & $\mathrm{ND}$ \\
\hline Selenium & $\mathrm{ND}$ \\
\hline Silver & $\mathrm{ND}$ \\
\hline Strontium-90 & $\mathrm{ND}$ \\
\hline
\end{tabular}

\begin{tabular}{|l|c|}
\hline 1009008-11 & B273B5 \\
\hline Analyte & Kd (L/g) \\
\hline Arsenic & ND \\
\hline Barium & ND \\
\hline Cadmium & ND \\
\hline Chromium (Total) & ND \\
\hline Chromium, Hexavalent & ND \\
\hline Lead & ND \\
\hline Selenium & ND \\
\hline Silver & ND \\
\hline Strontium-90 & ND \\
\hline
\end{tabular}




\section{Hexavalent Chromium/Soil - Quality Control \\ Environmental Science Laboratory}

\begin{tabular}{|lrrrrrrrrrrr}
\hline & & Reporting & & Spike & Source & & & $\%$ REC & & RPD \\
Analyte & Result & Limit & Units & Level & Result & $\%$ REC & Limits & RPD & Limit & Notes \\
\hline
\end{tabular}

Batch 0J14008 - Hexavalent Chromium Digest

Blank (0J14008-BLK1)

Chromium, Hexavalent Prepared \& Analyzed: 10/14/10

LCS (0J14008-BS1)

Chromium, Hexavalent

$<2.50 \mathrm{E} 0 \quad 2.50 \mathrm{E} 0 \quad \mathrm{ug} / \mathrm{g}$ wet

Prepared \& Analyzed: 10/14/10

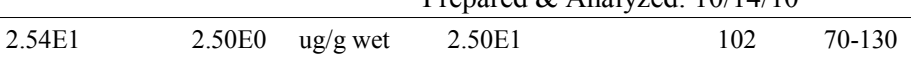

Duplicate (0J14008-DUP1)

Source: 1009008-01 Prepared \& Analyzed: 10/14/10

Chromium, Hexavalent

$<6.66 \mathrm{E}-1 \quad 6.66 \mathrm{E}-1 \quad \mathrm{ug} / \mathrm{g}$ dry

ND

35

Batch 1A14001 - Hexavalent Chromium Digest

Blank (1A14001-BLK1)

Prepared \& Analyzed: 01/13/11

Chromium, Hexavalent

$<5.00 \mathrm{E}-2 \quad 5.00 \mathrm{E}-2 \quad \mathrm{ug} / \mathrm{g}$ wet

LCS (1A14001-BS1)

Prepared \& Analyzed: 01/13/11

Chromium, Hexavalent

4.71E-1

$5.00 \mathrm{E}-2 \quad \mathrm{ug} / \mathrm{g}$ wet $\quad 5.00 \mathrm{E}-1$

$80-120$

Duplicate (1A14001-DUP1)

Source: 1009008-10 Prepared \& Analyzed: 01/13/11

Chromium, Hexavalent

$<6.92 \mathrm{E}-1 \quad 6.92 \mathrm{E}-1 \quad \mathrm{ug} / \mathrm{g}$ dry

ND 


\section{Hexavalent Chromium/1:1 Water Extract - Quality Control}

Environmental Science Laboratory

\begin{tabular}{|lrrrrrrrrrrr}
\hline & & Reporting & & Spike & Source & & & \%REC & & RPD & \\
Analyte & Result & Limit & Units & Level & Result & $\%$ REC & Limits & RPD & Limit & Notes \\
\hline
\end{tabular}

\section{Batch 0J11006 - 1:1 Water Extract (Cr6)}

Blank (0J11006-BLK1)

Chromium, Hexavalent

Prepared: 10/08/10 Analyzed: 10/11/10

LCS (0J11006-BS1)

Chromium, Hexavalent

$<5.00 \mathrm{E}-2 \quad 5.00 \mathrm{E}-2 \quad$ ug/g wet

Prepared: 10/08/10 Analyzed: 10/11/10

Duplicate (0J11006-DUP1)

Chromium, Hexavalent

Source: 1009008-01

Prepared: 10/08/10 Analyzed: 10/11/10

$<2.92 \mathrm{E}-2 \quad 2.92 \mathrm{E}-2 \quad \mathrm{ug} / \mathrm{g}$ dry

ND

Batch 1A06002 - 1:1 Water Extract (Cr6)

Blank (1A06002-BLK1)

Chromium, Hexavalent

LCS (1A06002-BS1)

Chromium, Hexavalent

Duplicate (1A06002-DUP1)

Chromium, Hexavalent
Prepared \& Analyzed: 01/07/11

$<5.00 \mathrm{E}-2 \quad 5.00 \mathrm{E}-2 \quad \mathrm{ug} / \mathrm{g}$ wet

Prepared \& Analyzed: 01/07/11

$\begin{array}{llllll}5.17 \mathrm{E}-1 & \text { N/A } & \mathrm{ug} / \mathrm{mL} & 5.00 \mathrm{E}-1 & 103 & 70-130\end{array}$

Source: 1009008-10 Prepared \& Analyzed: 01/07/11

$<3.88 \mathrm{E}-2 \quad 3.88 \mathrm{E}-2 \mathrm{ug} / \mathrm{g}$ dry $\quad$ ND

35 
Hexavalent Chromium/1:2.5 Water Extract - Quality Control

Environmental Science Laboratory

\begin{tabular}{|lrrrrrrrrrrr}
\hline & & Reporting & & Spike & Source & & & $\%$ REC & & RPD \\
Analyte & Result & Limit & Units & Level & Result & $\%$ REC & Limits & RPD & Limit & Notes \\
\hline
\end{tabular}

Batch 0J12002 - 1:2.5 Water Extract (Cr6)

Blank (0J12002-BLK1)

Chromium, Hexavalent

Prepared: 11/28/10 Analyzed: 12/02/10

LCS (0J12002-BS1)

Chromium, Hexavalent

$<5.00 \mathrm{E}-2 \quad 5.00 \mathrm{E}-2 \quad \mathrm{ug} / \mathrm{g}$ wet

Prepared: 11/28/10 Analyzed: 12/02/10

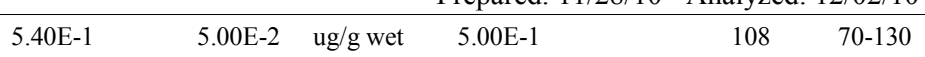

Duplicate (0J12002-DUP1)

Source: 1009008-01 Prepared: $11 / 28 / 10$ Analyzed: 12/02/10

Chromium, Hexavalent

$<1.02 \mathrm{E}-1 \quad 1.02 \mathrm{E}-1 \quad \mathrm{ug} / \mathrm{g}$ dry

ND

35

Post Spike (0J12002-PS1)

Source: 1009008-04

Prepared: 11/28/10 Analyzed: 12/02/10

Chromium, Hexavalent

$\begin{array}{lllllll}4.89 \mathrm{E}-1 & \mathrm{~N} / \mathrm{A} & \mathrm{ug} / \mathrm{mL} & 5.00 \mathrm{E}-1 & 7.00 \mathrm{E}-3 & 96.4 & 75-125\end{array}$

Batch 1A07001 - 1:2.5 Water Extract (Cr6)

Blank (1A07001-BLK1)

Prepared \& Analyzed: 01/07/11

Chromium, Hexavalent

$<5.00 \mathrm{E}-2 \quad 5.00 \mathrm{E}-2 \quad \mathrm{ug} / \mathrm{g}$ wet

LCS (1A07001-BS1)

Prepared \& Analyzed: 01/07/11

Chromium, Hexavalent

5.19E-1

$5.00 \mathrm{E}-2 \quad$ ug/g wet $\quad 5.00 \mathrm{E}-1$

$70-130$

Duplicate (1A07001-DUP1)

Source: 1009008-10 Prepared \& Analyzed: 01/07/11

Chromium, Hexavalent

$<1.05 \mathrm{E}-1 \quad 1.05 \mathrm{E}-1 \quad \mathrm{ug} / \mathrm{g}$ dry

ND 


\section{Hexavalent Chromium/1:5 Water Extract - Quality Control \\ Environmental Science Laboratory}

\begin{tabular}{|lrrrrrrrrrrr}
\hline & & Reporting & & Spike & Source & & & $\%$ REC & & RPD & \\
Analyte & Result & Limit & Units & Level & Result & $\%$ REC & Limits & RPD & Limit & Notes \\
\hline
\end{tabular}

\section{Batch 0J15001 - 1:5 Water Extract (Cr6)}

Blank (0J15001-BLK1)

Chromium, Hexavalent Prepared: 11/28/10 Analyzed: 12/02/10

LCS (0J15001-BS1)

Chromium, Hexavalent

$<5.00 \mathrm{E}-2 \quad 5.00 \mathrm{E}-2 \quad \mathrm{ug} / \mathrm{g}$ wet

Prepared: 11/28/10 Analyzed: $12 / 02 / 10$

Duplicate (0J15001-DUP1)

Chromium, Hexavalent Source: 1009008-01

Prepared: 11/28/10 Analyzed: 12/02/10

Post Spike (0J15001-PS1)

Chromium, Hexavalent

Source: 1009008-01

ND

Batch 1A07002 - 1:5 Water Extract (Cr6)

Blank (1A07002-BLK1)

Chromium, Hexavalent

LCS (1A07002-BS1)

Chromium, Hexavalent

Duplicate (1A07002-DUP1)

Chromium, Hexavalent

$71 \mathrm{E}-1-\mathrm{N} / \mathrm{A}$

Prepared: 11/28/10 Analyzed: 12/02/10

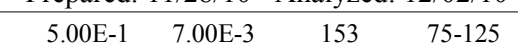

Prepared \& Analyzed: 01/07/11

$<5.00 \mathrm{E}-2 \quad 5.00 \mathrm{E}-2 \quad \mathrm{ug} / \mathrm{g}$ wet

Prepared \& Analyzed: 01/07/11

$\begin{array}{llllll}5.23 \mathrm{E}-1 & \mathrm{~N} / \mathrm{A} & \mathrm{ug} / \mathrm{mL} & 5.00 \mathrm{E}-1 & 105 & 70-130\end{array}$

Prepared \& Analyzed: 01/07/11

$<1.89 \mathrm{E}-1 \quad 1.89 \mathrm{E}-1 \quad \mathrm{ug} / \mathrm{g}$ dry 100900


Total Metals by PNNL-AGG-ICP-AES/1:1 Water Extract - Quality Control

Environmental Science Laboratory

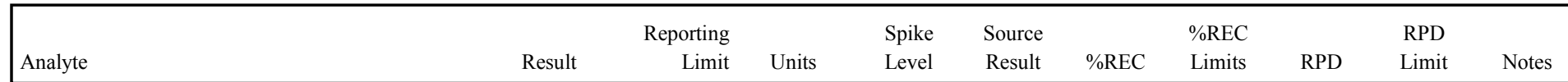

Batch 0J14005 - 1:1 Water Extract (ICP/ICPMS)

Blank (0J14005-BLK1)

\begin{tabular}{llll}
\hline Barium & $<1.24 \mathrm{E}-1 \quad 1.24 \mathrm{E}-1 \quad \mathrm{ug} / \mathrm{g}$ wet
\end{tabular}

Prepared: 10/06/10 Analyzed: 10/14/10

LCS (0J14005-BS1)

Barium

4.41E0 $1.24 \mathrm{E}-1 \quad$ ug/g wet $4.99 \mathrm{E} 0 \quad 88.4 \quad 80-120$

Duplicate (0J14005-DUP1)

Barium

Source: 1009008-01

Prepared: 10/06/10 Analyzed: 10/14/10

Post Spike (0J14005-PS1)

$\begin{array}{lllll}<1.16 \mathrm{E}-1 & 1.16 \mathrm{E}-1 & \text { ug/g dry } & \text { ND } & 35\end{array}$

Barium

Source: 1009008-01

Prepared \& Analyzed: 10/14/10

$\begin{array}{lllllll}2.72 \mathrm{E} 2 & \mathrm{~N} / \mathrm{A} & \mathrm{ug} / \mathrm{L} & 2.50 \mathrm{E} 2 & 8.87 \mathrm{E} 0 & 105 & 75-125\end{array}$

Batch 1A31003 - 1:1 Water Extract (ICP/ICPMS)

Blank (1A31003-BLK1)

Prepared: 01/05/11 Analyzed: 02/01/11

Barium

$<8.25 \mathrm{E}-2 \quad 8.25 \mathrm{E}-2 \quad \mathrm{ug} / \mathrm{g}$ wet

LCS (1A31003-BS1)

Prepared: 01/05/11 Analyzed: 02/01/11

Barium

$4.51 \mathrm{E} 0$

$8.25 \mathrm{E}-2 \quad \mathrm{ug} / \mathrm{g}$ wet $\quad 5.00 \mathrm{E} 0$

$90.2 \quad 80-120$

Duplicate (1A31003-DUP1)

Source: 1009008-10 Prepared: 01/05/11 Analyzed: 02/01/11

Barium

$<9.55 \mathrm{E}-2 \quad 9.55 \mathrm{E}-2 \quad \mathrm{ug} / \mathrm{g}$ dry

ND

35

Post Spike (1A31003-PS1)

Barium

Source: 1009008-10

Prepared \& Analyzed: 02/01/11

$\begin{array}{lllllll}2.76 \mathrm{E} 2 & \mathrm{~N} / \mathrm{A} & \text { ug/L } & 2.50 \mathrm{E} 2 & 2.95 \mathrm{E} 1 & 98.7 & 75-125\end{array}$ 
Total Metals by PNNL-AGG-ICP-AES/1:2.5 Water Extract - Quality Control

Environmental Science Laboratory

\begin{tabular}{|lrrrrrrrrrrr}
\hline & & Reporting & & Spike & Source & & \%REC & & RPD \\
Analyte & Result & Limit & Units & Level & Result & $\%$ REC & Limits & RPD & Limit & Notes \\
\hline
\end{tabular}

\section{Batch 0J14006 - 1:2.5 Water Extract (ICP/ICPMS)}

\begin{tabular}{|c|c|c|c|c|c|c|}
\hline \multirow{2}{*}{$\begin{array}{l}\text { Blank (0J14006-BLK1) } \\
\text { Barium }\end{array}$} & & & & Prepared: 10/06/10 & Analyzed: 12/02/10 & \\
\hline & $<1.24 \mathrm{E}-1$ & $1.24 \mathrm{E}-1$ & ug/g wet & & & \\
\hline \multicolumn{4}{|l|}{ LCS (0J14006-BS1) } & Prepared: 10/06/10 & Analyzed: $12 / 02 / 10$ & \\
\hline Barium & $4.83 \mathrm{E} 0$ & $1.24 \mathrm{E}-1$ & ug/g wet & $5.00 \mathrm{E} 0$ & $80-120$ & \\
\hline \multicolumn{2}{|l|}{ Duplicate (0J14006-DUP1) } & \multicolumn{2}{|c|}{ Source: 1009008-01 } & Prepared: 10/06/10 & \multirow[t]{2}{*}{ Analyzed: $12 / 02 / 10$} & \\
\hline Barium & $<2.96 \mathrm{E}-1$ & $2.96 \mathrm{E}-1$ & ug/g dry & ND & & 35 \\
\hline Post Spike (0J14006-PS1) & \multicolumn{3}{|c|}{ Source: 1009008-01 } & Prepared: 10/14/10 & Analyzed: $12 / 02 / 10$ & \\
\hline Barium & $2.65 \mathrm{E} 2$ & N/A & $\mathrm{ug} / \mathrm{L}$ & $5.30 \mathrm{E} 0$ & $75-125$ & \\
\hline \multicolumn{7}{|c|}{ Batch 1A31004 - 1:2.5 Water Extract (ICP/ICPMS) } \\
\hline \multicolumn{4}{|l|}{ Blank (1A31004-BLK1) } & \multirow[t]{2}{*}{ Prepared: 01/05/11 } & \multirow[t]{2}{*}{ Analyzed: 02/01/11 } & \\
\hline Barium & $<8.25 \mathrm{E}-2$ & $8.25 \mathrm{E}-2$ & ug/g wet & & & \\
\hline \multicolumn{4}{|l|}{ LCS (1A31004-BS1) } & Prepared: 01/05/11 & Analyzed: $02 / 01 / 11$ & \\
\hline Barium & 4.47E0 & $8.25 \mathrm{E}-2$ & ug/g wet & 4.99E0 & $80-120$ & \\
\hline \multicolumn{2}{|l|}{ Duplicate (1A31004-DUP1) } & \multicolumn{2}{|c|}{ Source: $\mathbf{1 0 0 9 0 0 8 - 1 0}$} & Prepared: 01/05/11 & Analyzed: $02 / 01 / 11$ & \\
\hline Barium & $<2.06 \mathrm{E}-1$ & $2.06 \mathrm{E}-1$ & ug/g dry & ND & & 35 \\
\hline Post Spike (1A31004-PS1) & \multicolumn{3}{|c|}{ Source: 1009008-10 } & \multicolumn{2}{|c|}{ Prepared \& Analyzed: 02/01/11 } & \\
\hline Barium & $2.62 \mathrm{E} 2$ & N/A & $\mathrm{ug} / \mathrm{L}$ & $2.50 \mathrm{E} 2$ & $75-125$ & \\
\hline
\end{tabular}


Total Metals by PNNL-AGG-ICP-AES/1:5 Water Extract - Quality Control

Environmental Science Laboratory

\begin{tabular}{|lrrrrrrrrrrrr}
\hline & & Reporting & & Spike & Source & & & $\%$ REC & & RPD \\
Analyte & Result & Limit & Units & Level & Result & $\%$ REC & Limits & RPD & Limit & Notes \\
\hline
\end{tabular}

Batch 0J14007 - 1:5 Water Extract (ICP/ICPMS)

Blank (0J14007-BLK1)

\begin{tabular}{llll}
\hline Barium & $<1.24 \mathrm{E}-1 \quad 1.24 \mathrm{E}-1 \quad \mathrm{ug} / \mathrm{g}$ wet
\end{tabular}

Prepared: 10/06/10 Analyzed: 12/02/10

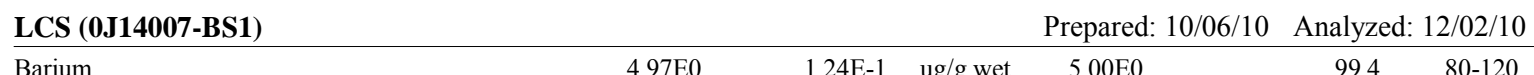

$\begin{array}{lll} & -24 \mathrm{E}-1-120 & -120\end{array}$

Duplicate (0J14007-DUP1)

Source: 1009008-01 Prepared: 10/06/10 Analyzed: 12/02/10

Barium

$<5.90 \mathrm{E}-1 \quad 5.90 \mathrm{E}-1 \quad$ ug/g dry $\quad$ ND

35

Post Spike (0J14007-PS1)

Source: 1009008-01 Prepared: 10/14/10 Analyzed: 12/02/10

Barium

$\begin{array}{lllllll}2.55 \mathrm{E} 2 & \mathrm{~N} / \mathrm{A} & \mathrm{ug} / \mathrm{L} & 2.50 \mathrm{E} 2 & 3.89 \mathrm{E} 0 & 100 & 75-125\end{array}$

Batch 1A31005 - 1:5 Water Extract (ICP/ICPMS)

Blank (1A31005-BLK1)

Prepared: 01/05/11 Analyzed: 02/01/11

Barium

$<8.25 \mathrm{E}-2 \quad 8.25 \mathrm{E}-2 \quad \mathrm{ug} / \mathrm{g}$ wet

LCS (1A31005-BS1)

Prepared: 01/05/11 Analyzed: 02/01/11

Barium

$4.57 \mathrm{E} 0$

$8.25 \mathrm{E}-2 \quad \mathrm{ug} / \mathrm{g}$ wet $\quad 5.00 \mathrm{E} 0$

$80-120$

Duplicate (1A31005-DUP1)

Source: 1009008-10 Prepared: 01/05/11 Analyzed: 02/01/11

Barium

$<3.43 \mathrm{E}-1 \quad 3.43 \mathrm{E}-1 \quad \mathrm{ug} / \mathrm{g}$ dry

ND

35

Post Spike (1A31005-PS1)

Source: 1009008-10 Prepared \& Analyzed: 02/01/11

Barium

$\begin{array}{lllllll}2.57 \mathrm{E} 2 & \mathrm{~N} / \mathrm{A} & \mathrm{ug} / \mathrm{L} & 2.50 \mathrm{E} 2 & 1.11 \mathrm{E} 1 & 98.4 & 75-125\end{array}$


Total Metals by PNNL-AGG-ICP-AES/Acid Extract - Quality Control

Environmental Science Laboratory

\begin{tabular}{|lrrrrrrrrrrrr}
\hline & & Reporting & & Spike & Source & & & $\%$ REC & & RPD \\
Analyte & Result & Limit & Units & Level & Result & $\%$ REC & Limits & RPD & Limit & Notes \\
\hline
\end{tabular}

\section{Batch 0J14004 - ASTM D 5198 (ICP/ICPMS)}

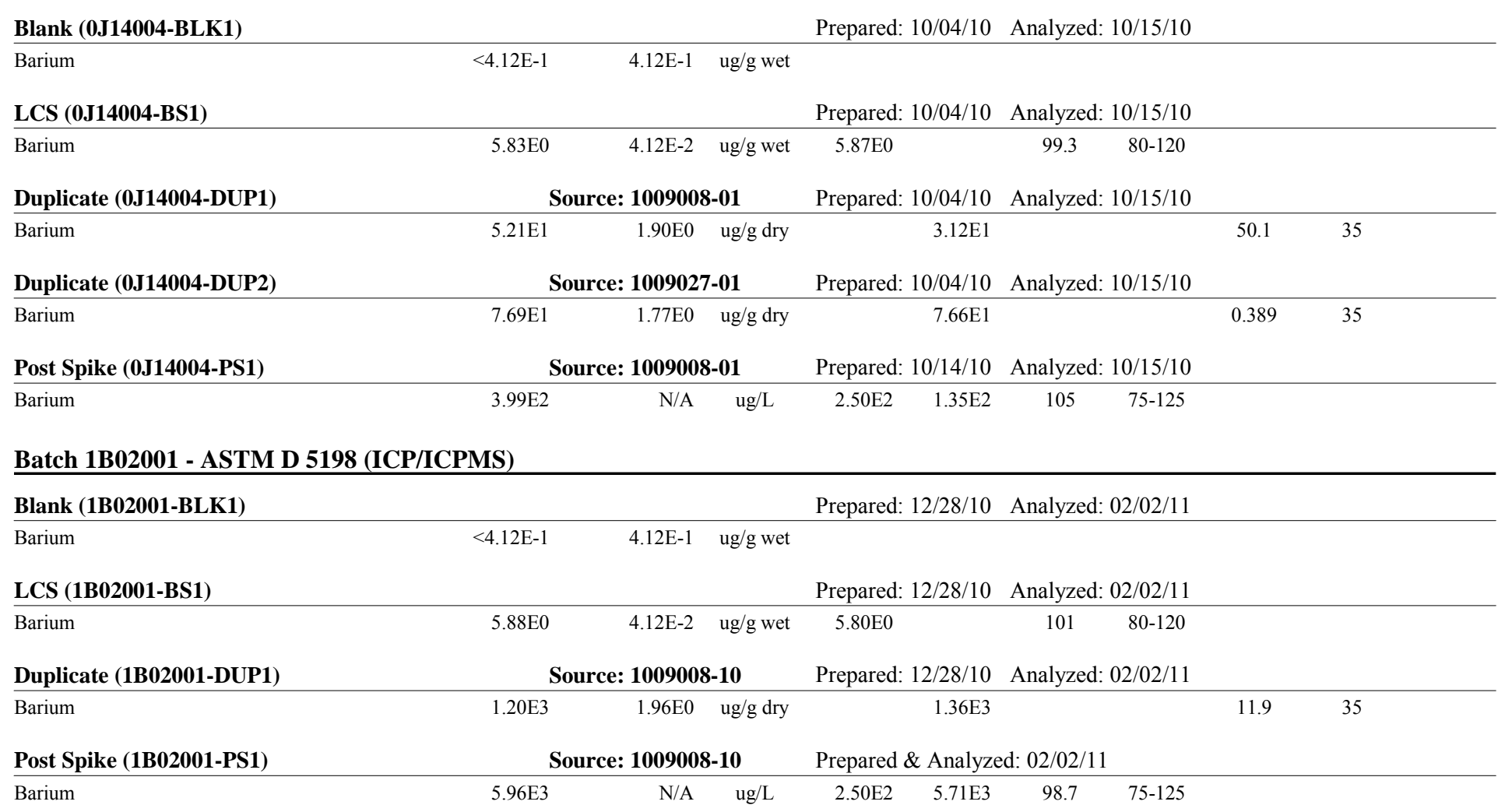


RCRA Metals By PNNL-AGG-415/1:1 Water Extract - Quality Control

Environmental Science Laboratory

\begin{tabular}{|lrrrrrrrrrrr}
\hline & & Reporting & & Spike & Source & & & $\%$ REC & & RPD & \\
Analyte & Result & Limit & Units & Level & Result & $\%$ REC & Limits & RPD & Limit & Notes \\
\hline
\end{tabular}

Batch 0J11002 - 1:1 Water Extract (ICP/ICPMS)

\begin{tabular}{|c|c|c|c|c|c|c|c|c|c|}
\hline \multirow{2}{*}{$\begin{array}{l}\text { Blank (0J11002-BLK1) } \\
\text { Chromium }\end{array}$} & & & & \multicolumn{2}{|c|}{ Prepared: 10/06/10 } & \multicolumn{2}{|c|}{ Analyzed: 10/11/10 } & & \\
\hline & $<3.44 \mathrm{E}-3$ & $3.44 \mathrm{E}-3$ & ug/g wet & & & & & & \\
\hline Arsenic & $<2.84 \mathrm{E}-3$ & $2.84 \mathrm{E}-3$ & $"$ & & & & & & \\
\hline Selenium & $<7.92 \mathrm{E}-3$ & $7.92 \mathrm{E}-3$ & $"$ & & & & & & \\
\hline Silver & $<3.13 \mathrm{E}-3$ & $3.13 \mathrm{E}-3$ & $"$ & & & & & & \\
\hline Cadmium & $<5.73 \mathrm{E}-4$ & $5.73 \mathrm{E}-4$ & $"$ & & & & & & \\
\hline Lead & $<1.16 \mathrm{E}-3$ & $1.16 \mathrm{E}-3$ & $"$ & & & & & & \\
\hline LCS (0J11002-BS1) & \multicolumn{5}{|c|}{ Prepared: 10/06/10 } & \multicolumn{2}{|c|}{ Analyzed: 10/11/10 } & & \\
\hline Chromium & $4.07 \mathrm{E} 0$ & $3.44 \mathrm{E}-1$ & ug/g wet & 4.99E0 & & 81.5 & $80-120$ & & \\
\hline Arsenic & $4.20 \mathrm{E} 0$ & $2.84 \mathrm{E}-1$ & $"$ & $4.99 \mathrm{E} 0$ & & 84.1 & $80-120$ & & \\
\hline Selenium & $4.21 \mathrm{E} 0$ & 7.92E-1 & $"$ & 4.99E0 & & 84.3 & $80-120$ & & \\
\hline Silver & 4.10E0 & $3.13 \mathrm{E}-1$ & $"$ & 4.99E0 & & 82.1 & $80-120$ & & \\
\hline Cadmium & 4.17E0 & $5.73 \mathrm{E}-2$ & $"$ & $4.99 \mathrm{E} 0$ & & 83.5 & $80-120$ & & \\
\hline Lead & $4.04 \mathrm{E} 0$ & $1.16 \mathrm{E}-1$ & $"$ & 4.99E0 & & 81.0 & $80-120$ & & \\
\hline Duplicate (0J11002-DUP1) & \multicolumn{3}{|c|}{ Source: 1009008-01 } & \multicolumn{2}{|c|}{ Prepared: 10/06/10 } & \multicolumn{2}{|c|}{ Analyzed: 10/11/10 } & & \\
\hline Chromium & $<3.22 \mathrm{E}-3$ & $3.22 \mathrm{E}-3$ & ug/g dry & & ND & & & & 35 \\
\hline Arsenic & $9.45 \mathrm{E}-3$ & $2.65 \mathrm{E}-3$ & $"$ & & $9.25 \mathrm{E}-3$ & & & 2.20 & 35 \\
\hline Selenium & $1.69 \mathrm{E}-2$ & $7.40 \mathrm{E}-3$ & $"$ & & $1.83 \mathrm{E}-2$ & & & 7.89 & 35 \\
\hline Silver & $<2.93 \mathrm{E}-3$ & $2.93 \mathrm{E}-3$ & $"$ & & ND & & & & 35 \\
\hline Cadmium & $<5.36 \mathrm{E}-4$ & $5.36 \mathrm{E}-4$ & $"$ & & ND & & & & 35 \\
\hline Lead & $<1.09 \mathrm{E}-3$ & $1.09 \mathrm{E}-3$ & $"$ & & ND & & & & 35 \\
\hline Post Spike (0J11002-PS1) & \multicolumn{3}{|c|}{ Source: 1009008-06 } & \multicolumn{4}{|c|}{ Prepared \& Analyzed: 10/11/10 } & & \\
\hline Chromium & 4.94E0 & N/A & $\mathrm{ug} / \mathrm{L}$ & $5.00 \mathrm{E} 0$ & $3.29 \mathrm{E}-2$ & 98.2 & $75-125$ & & \\
\hline Arsenic & $7.10 \mathrm{E} 0$ & N/A & $"$ & $5.00 \mathrm{E} 0$ & $1.70 \mathrm{E} 0$ & 108 & $75-125$ & & \\
\hline Selenium & $5.76 \mathrm{E} 0$ & N/A & $"$ & $5.00 \mathrm{E} 0$ & $1.14 \mathrm{E}-1$ & 113 & $75-125$ & & \\
\hline Silver & $4.85 \mathrm{E} 0$ & N/A & $"$ & $5.00 \mathrm{E} 0$ & ND & 97 & $75-125$ & & \\
\hline Cadmium & $5.09 \mathrm{E} 0$ & N/A & $"$ & $5.00 \mathrm{E} 0$ & $3.95 \mathrm{E}-3$ & 102 & $75-125$ & & \\
\hline Lead & $4.92 \mathrm{E} 0$ & N/A & $"$ & $5.00 \mathrm{E} 0$ & $1.24 \mathrm{E}-2$ & 98.1 & $75-125$ & & \\
\hline
\end{tabular}


RCRA Metals By PNNL-AGG-415/1:1 Water Extract - Quality Control

Environmental Science Laboratory

\begin{tabular}{|lrrrrrrrrrrr}
\hline & & Reporting & & Spike & Source & & & $\%$ REC & & RPD & \\
Analyte & Result & Limit & Units & Level & Result & $\%$ REC & Limits & RPD & Limit & Notes \\
\hline
\end{tabular}

Batch 1A17001 - 1:1 Water Extract (ICP/ICPMS)

\begin{tabular}{|c|c|c|c|c|c|c|c|c|}
\hline \multirow{2}{*}{$\begin{array}{l}\text { Blank (1A17001-BLK1) } \\
\text { Chromium }\end{array}$} & \multicolumn{8}{|c|}{ Prepared \& Analyzed: 01/17/11 } \\
\hline & $<3.44 \mathrm{E}-3$ & $3.44 \mathrm{E}-3$ & ug/g wet & & & & & \\
\hline Arsenic & $<1.42 \mathrm{E}-2$ & $1.42 \mathrm{E}-2$ & $"$ & & & & & \\
\hline Selenium & $<7.92 \mathrm{E}-3$ & $7.92 \mathrm{E}-3$ & $"$ & & & & & \\
\hline Silver & $<3.13 \mathrm{E}-3$ & $3.13 \mathrm{E}-3$ & $"$ & & & & & \\
\hline Cadmium & $<5.73 \mathrm{E}-4$ & $5.73 \mathrm{E}-4$ & $"$ & & & & & \\
\hline Lead & $<1.16 \mathrm{E}-3$ & $1.16 \mathrm{E}-3$ & $"$ & & & & & \\
\hline LCS (1A17001-BS1) & \multicolumn{8}{|c|}{ Prepared \& Analyzed: 01/17/11 } \\
\hline Chromium & $4.49 \mathrm{E} 0$ & $3.44 \mathrm{E}-1$ & ug/g wet & $5.00 \mathrm{E} 0$ & 89.8 & $80-120$ & & \\
\hline Arsenic & $4.61 \mathrm{E} 0$ & $2.84 \mathrm{E}-1$ & $"$ & $5.00 \mathrm{E} 0$ & 92.2 & $80-120$ & & \\
\hline Selenium & $4.35 \mathrm{E} 0$ & $7.92 \mathrm{E}-1$ & $"$ & $5.00 \mathrm{E} 0$ & 87.1 & $80-120$ & & \\
\hline Silver & 4.57E0 & $3.13 \mathrm{E}-1$ & $"$ & $5.00 \mathrm{E} 0$ & 91.4 & $80-120$ & & \\
\hline Cadmium & 4.49E0 & $5.73 \mathrm{E}-2$ & $"$ & $5.00 \mathrm{E} 0$ & 89.9 & $80-120$ & & \\
\hline Lead & $4.60 \mathrm{E} 0$ & $1.16 \mathrm{E}-1$ & $"$ & $5.00 \mathrm{E} 0$ & 92.1 & $80-120$ & & \\
\hline Duplicate (1A17001-DUP1) & \multicolumn{3}{|c|}{ Source: $1009008-10$} & \multicolumn{5}{|c|}{ Prepared \& Analyzed: 01/17/11 } \\
\hline Chromium & $2.14 \mathrm{E}-2$ & $3.99 \mathrm{E}-3$ & ug/g dry & & & & 0.429 & 35 \\
\hline Arsenic & $5.33 \mathrm{E}-2$ & $1.64 \mathrm{E}-2$ & $"$ & & & & 29.3 & 35 \\
\hline Selenium & $<9.16 \mathrm{E}-3$ & $9.16 \mathrm{E}-3$ & $"$ & & & & & 35 \\
\hline Silver & $<3.62 \mathrm{E}-3$ & $3.62 \mathrm{E}-3$ & $"$ & & & & & 35 \\
\hline Cadmium & $<6.63 \mathrm{E}-4$ & $6.63 \mathrm{E}-4$ & $"$ & & & & & 35 \\
\hline Lead & $<1.34 \mathrm{E}-3$ & $1.34 \mathrm{E}-3$ & $"$ & & & & & 35 \\
\hline
\end{tabular}


RCRA Metals By PNNL-AGG-415/1:2.5 Water Extract - Quality Control

Environmental Science Laboratory

\begin{tabular}{|lrrrrrrrrrrrr}
\hline & & Reporting & & Spike & Source & & & $\%$ REC & & RPD \\
Analyte & Result & Limit & Units & Level & Result & $\%$ REC & Limits & RPD & Limit & Notes \\
\hline
\end{tabular}

Batch 0J11003 - 1:2.5 Water Extract (ICP/ICPMS)

\begin{tabular}{|c|c|c|c|c|c|c|c|c|c|}
\hline \multirow{2}{*}{$\frac{\text { Blank (0J11003-BLK1) }}{\text { Chromium }}$} & \multirow[b]{2}{*}{$<3.44 \mathrm{E}-3$} & \multirow[b]{2}{*}{$3.44 \mathrm{E}-3$} & \multirow{3}{*}{$\begin{array}{l}\text { ug/g wet } \\
\text { " }\end{array}$} & \multicolumn{2}{|c|}{ Prepared: 10/06/10 } & \multicolumn{2}{|c|}{ Analyzed: 12/06/10 } & & \\
\hline & & & & & & & & & \\
\hline Arsenic & $<2.84 \mathrm{E}-3$ & $2.84 \mathrm{E}-3$ & & & & & & & \\
\hline Selenium & $<7.92 \mathrm{E}-3$ & $7.92 \mathrm{E}-3$ & $"$ & & & & & & \\
\hline Silver & $<3.13 \mathrm{E}-3$ & $3.13 \mathrm{E}-3$ & $"$ & & & & & & \\
\hline Cadmium & $<5.73 \mathrm{E}-4$ & $5.73 \mathrm{E}-4$ & $"$ & & & & & & \\
\hline Lead & $<1.16 \mathrm{E}-3$ & $1.16 \mathrm{E}-3$ & $"$ & & & & & & \\
\hline LCS (0J11003-BS1) & \multicolumn{5}{|c|}{ Prepared: 10/06/10 } & \multicolumn{2}{|c|}{ Analyzed: 12/06/10 } & & \\
\hline Chromium & $4.59 \mathrm{E} 0$ & $3.44 \mathrm{E}-1$ & ug/g wet & $5.00 \mathrm{E} 0$ & & 91.9 & $80-120$ & & \\
\hline Arsenic & 4.64E0 & $2.84 \mathrm{E}-1$ & $"$ & $5.00 \mathrm{E} 0$ & & 92.8 & $80-120$ & & \\
\hline Selenium & 4.69E0 & $7.92 \mathrm{E}-1$ & $"$ & $5.00 \mathrm{E} 0$ & & 93.9 & $80-120$ & & \\
\hline Silver & $4.71 \mathrm{E} 0$ & $3.13 \mathrm{E}-1$ & $"$ & $5.00 \mathrm{E} 0$ & & 94.2 & $80-120$ & & \\
\hline Cadmium & $4.78 \mathrm{E} 0$ & $5.73 \mathrm{E}-2$ & $"$ & $5.00 \mathrm{E} 0$ & & 95.7 & $80-120$ & & \\
\hline Lead & 4.82E0 & $1.16 \mathrm{E}-1$ & $"$ & $5.00 \mathrm{E} 0$ & & 96.4 & $80-120$ & & \\
\hline Duplicate (0J11003-DUP1) & \multicolumn{3}{|c|}{ Source: 1009008-01 } & \multicolumn{2}{|c|}{ Prepared: 10/06/10 } & \multicolumn{2}{|c|}{ Analyzed: 12/06/10 } & & \\
\hline Chromium & $<8.25 \mathrm{E}-3$ & $8.25 \mathrm{E}-3$ & ug/g dry & & ND & & & & 35 \\
\hline Arsenic & $9.86 \mathrm{E}-3$ & $6.79 \mathrm{E}-3$ & $"$ & & $8.83 \mathrm{E}-3$ & & & 11.0 & 35 \\
\hline Selenium & $<1.89 \mathrm{E}-2$ & $1.89 \mathrm{E}-2$ & $"$ & & ND & & & & 35 \\
\hline Silver & $<7.49 \mathrm{E}-3$ & $7.49 \mathrm{E}-3$ & $"$ & & ND & & & & 35 \\
\hline Cadmium & $<1.37 \mathrm{E}-3$ & $1.37 \mathrm{E}-3$ & $"$ & & ND & & & & 35 \\
\hline Lead & $<2.78 \mathrm{E}-3$ & $2.78 \mathrm{E}-3$ & $"$ & & ND & & & & 35 \\
\hline Post Spike (0J11003-PS1) & \multicolumn{3}{|c|}{ Source: 1009008-06 } & \multicolumn{2}{|c|}{ Prepared: 10/11/10 } & \multicolumn{2}{|c|}{ Analyzed: 12/06/10 } & & \\
\hline Chromium & $4.83 \mathrm{E} 0$ & N/A & $\mathrm{ug} / \mathrm{L}$ & $5.00 \mathrm{E} 0$ & ND & 96.7 & $75-125$ & & \\
\hline Arsenic & 5.94E0 & N/A & $"$ & $5.00 \mathrm{E} 0$ & $1.03 \mathrm{E} 0$ & 98.2 & $75-125$ & & \\
\hline Selenium & $5.48 \mathrm{E} 0$ & N/A & $"$ & $5.00 \mathrm{E} 0$ & $2.20 \mathrm{E}-1$ & 105 & $75-125$ & & \\
\hline Silver & $4.66 \mathrm{E} 0$ & N/A & $"$ & $5.00 \mathrm{E} 0$ & $1.57 \mathrm{E}-4$ & 93.3 & $75-125$ & & \\
\hline Cadmium & 4.90E0 & N/A & $"$ & $5.00 \mathrm{E} 0$ & ND & 97.9 & $75-125$ & & \\
\hline Lead & $5.11 \mathrm{E} 0$ & N/A & $"$ & $5.00 \mathrm{E} 0$ & $6.33 \mathrm{E}-3$ & 102 & $75-125$ & & \\
\hline
\end{tabular}


RCRA Metals By PNNL-AGG-415/1:2.5 Water Extract - Quality Control

Environmental Science Laboratory

\begin{tabular}{|lrrrrrrrrrrr}
\hline & & Reporting & & Spike & Source & & & $\%$ REC & & RPD & \\
Analyte & Result & Limit & Units & Level & Result & $\%$ REC & Limits & RPD & Limit & Notes \\
\hline
\end{tabular}

Batch 1A17002 - 1:2.5 Water Extract (ICP/ICPMS)

\begin{tabular}{|c|c|c|c|c|c|c|c|c|}
\hline \multirow{2}{*}{$\begin{array}{l}\text { Blank (1A17002-BLK1) } \\
\text { Chromium }\end{array}$} & \multicolumn{8}{|c|}{ Prepared \& Analyzed: 01/17/11 } \\
\hline & $<3.44 \mathrm{E}-3$ & $3.44 \mathrm{E}-3$ & ug/g wet & & & & & \\
\hline Arsenic & $<1.42 \mathrm{E}-2$ & $1.42 \mathrm{E}-2$ & $"$ & & & & & \\
\hline Selenium & $<7.92 \mathrm{E}-3$ & $7.92 \mathrm{E}-3$ & $"$ & & & & & \\
\hline Silver & $<3.13 \mathrm{E}-3$ & $3.13 \mathrm{E}-3$ & $"$ & & & & & \\
\hline Cadmium & $<5.73 \mathrm{E}-4$ & $5.73 \mathrm{E}-4$ & $"$ & & & & & \\
\hline Lead & $<1.16 \mathrm{E}-3$ & $1.16 \mathrm{E}-3$ & $"$ & & & & & \\
\hline LCS (1A17002-BS1) & \multicolumn{8}{|c|}{ Prepared \& Analyzed: 01/17/11 } \\
\hline Chromium & $4.53 \mathrm{E} 0$ & $3.44 \mathrm{E}-1$ & ug/g wet & $4.99 \mathrm{E} 0$ & 90.7 & $80-120$ & & \\
\hline Arsenic & 4.64E0 & $2.84 \mathrm{E}-1$ & $"$ & 4.99E0 & 93.0 & $80-120$ & & \\
\hline Selenium & $4.60 \mathrm{E} 0$ & $7.92 \mathrm{E}-1$ & $"$ & 4.99E0 & 92.1 & $80-120$ & & \\
\hline Silver & $4.52 \mathrm{E} 0$ & $3.13 \mathrm{E}-1$ & $"$ & 4.99E0 & 90.6 & $80-120$ & & \\
\hline Cadmium & 4.46Е0 & $5.73 \mathrm{E}-2$ & $"$ & 4.99E0 & 89.4 & $80-120$ & & \\
\hline Lead & $4.53 \mathrm{E} 0$ & $1.16 \mathrm{E}-1$ & $"$ & $4.99 \mathrm{E} 0$ & 90.7 & $80-120$ & & \\
\hline Duplicate (1A17002-DUP1) & \multicolumn{3}{|c|}{ Source: 1009008-10 } & \multicolumn{5}{|c|}{ Prepared \& Analyzed: 01/17/11 } \\
\hline Chromium & $2.22 \mathrm{E}-2$ & $8.58 \mathrm{E}-3$ & ug/g dry & & & & 3.83 & 35 \\
\hline Arsenic & $9.64 \mathrm{E}-2$ & $3.53 \mathrm{E}-2$ & $"$ & & & & 8.39 & 35 \\
\hline Selenium & $<1.97 \mathrm{E}-2$ & $1.97 \mathrm{E}-2$ & $"$ & & & & & 35 \\
\hline Silver & $<7.79 \mathrm{E}-3$ & $7.79 \mathrm{E}-3$ & $"$ & & & & & 35 \\
\hline Cadmium & $<1.43 \mathrm{E}-3$ & $1.43 \mathrm{E}-3$ & $"$ & & & & & 35 \\
\hline Lead & $<2.89 \mathrm{E}-3$ & $2.89 \mathrm{E}-3$ & $"$ & & & & & 35 \\
\hline
\end{tabular}


RCRA Metals By PNNL-AGG-415/1:5 Water Extract - Quality Control

Environmental Science Laboratory

\begin{tabular}{|lrrrrrrrrrrr}
\hline & & Reporting & & Spike & Source & & & $\%$ REC & & RPD & \\
Analyte & Result & Limit & Units & Level & Result & $\%$ REC & Limits & RPD & Limit & Notes \\
\hline
\end{tabular}

Batch 0J11004 - 1:5 Water Extract (ICP/ICPMS)

\begin{tabular}{|c|c|c|c|c|c|c|c|c|}
\hline \multirow{2}{*}{$\begin{array}{l}\text { Blank (0J11004-BLK1) } \\
\text { Chromium }\end{array}$} & & & & \multicolumn{2}{|c|}{ Prepared: 10/06/10 } & \multicolumn{2}{|c|}{ Analyzed: 12/06/10 } & \\
\hline & $<3.44 \mathrm{E}-3$ & $3.44 \mathrm{E}-3$ & ug/g wet & & & & & \\
\hline Arsenic & $<2.84 \mathrm{E}-3$ & $2.84 \mathrm{E}-3$ & $"$ & & & & & \\
\hline Selenium & $<7.92 \mathrm{E}-3$ & $7.92 \mathrm{E}-3$ & $"$ & & & & & \\
\hline Silver & $<3.13 \mathrm{E}-3$ & $3.13 \mathrm{E}-3$ & $"$ & & & & & \\
\hline Cadmium & $<5.73 \mathrm{E}-4$ & $5.73 \mathrm{E}-4$ & $"$ & & & & & \\
\hline Lead & $<1.16 \mathrm{E}-3$ & $1.16 \mathrm{E}-3$ & $"$ & & & & & \\
\hline \multicolumn{4}{|l|}{ LCS (0J11004-BS1) } & \multicolumn{2}{|c|}{ Prepared: 10/06/10 } & \multicolumn{2}{|c|}{ Analyzed: 12/06/10 } & \\
\hline Chromium & $4.58 \mathrm{E} 0$ & $3.44 \mathrm{E}-1$ & ug/g wet & $5.00 \mathrm{E} 0$ & & 91.6 & $80-120$ & \\
\hline Arsenic & $4.52 \mathrm{E} 0$ & $2.84 \mathrm{E}-1$ & $"$ & $5.00 \mathrm{E} 0$ & & 90.4 & $80-120$ & \\
\hline Selenium & $4.67 \mathrm{E} 0$ & $7.92 \mathrm{E}-1$ & $"$ & $5.00 \mathrm{E} 0$ & & 93.3 & $80-120$ & \\
\hline Silver & $4.60 \mathrm{E} 0$ & $3.13 \mathrm{E}-1$ & $"$ & $5.00 \mathrm{E} 0$ & & 92.1 & $80-120$ & \\
\hline Cadmium & 4.70E0 & $5.73 \mathrm{E}-2$ & $"$ & $5.00 \mathrm{E} 0$ & & 93.9 & $80-120$ & \\
\hline Lead & 4.75E0 & $1.16 \mathrm{E}-1$ & $"$ & $5.00 \mathrm{E} 0$ & & 94.9 & $80-120$ & \\
\hline Duplicate (0J11004-DUP1) & \multicolumn{3}{|c|}{ Source: 1009008-01 } & \multicolumn{2}{|c|}{ Prepared: 10/06/10 } & \multicolumn{2}{|c|}{ Analyzed: 12/06/10 } & \\
\hline Chromium & $<1.64 \mathrm{E}-2$ & $1.64 \mathrm{E}-2$ & ug/g dry & & ND & & & 35 \\
\hline Arsenic & $<1.35 \mathrm{E}-2$ & $1.35 \mathrm{E}-2$ & $"$ & & ND & & & 35 \\
\hline Selenium & $<3.77 \mathrm{E}-2$ & $3.77 \mathrm{E}-2$ & $"$ & & ND & & & 35 \\
\hline Silver & $<1.49 \mathrm{E}-2$ & $1.49 \mathrm{E}-2$ & $"$ & & ND & & & 35 \\
\hline Cadmium & $<2.73 \mathrm{E}-3$ & $2.73 \mathrm{E}-3$ & $"$ & & ND & & & 35 \\
\hline Lead & $<5.54 \mathrm{E}-3$ & $5.54 \mathrm{E}-3$ & $"$ & & ND & & & 35 \\
\hline Post Spike (0J11004-PS1) & \multicolumn{3}{|c|}{ Source: 1009008-06 } & \multicolumn{2}{|c|}{ Prepared: 10/11/10 } & \multicolumn{2}{|c|}{ Analyzed: 12/06/10 } & \\
\hline Chromium & $4.75 \mathrm{E} 0$ & N/A & $\mathrm{ug} / \mathrm{L}$ & $5.00 \mathrm{E} 0$ & ND & 95.5 & $75-125$ & \\
\hline Arsenic & $5.43 \mathrm{E} 0$ & N/A & $"$ & $5.00 \mathrm{E} 0$ & $8.01 \mathrm{E}-1$ & 92.6 & $75-125$ & \\
\hline Selenium & 4.94E0 & N/A & $"$ & $5.00 \mathrm{E} 0$ & $3.15 \mathrm{E}-2$ & 98.1 & $75-125$ & \\
\hline Silver & $4.60 \mathrm{E} 0$ & N/A & $"$ & $5.00 \mathrm{E} 0$ & $1.20 \mathrm{E}-2$ & 91.8 & $75-125$ & \\
\hline Cadmium & 4.94E0 & N/A & $"$ & $5.00 \mathrm{E} 0$ & $5.09 \mathrm{E}-4$ & 98.7 & $75-125$ & \\
\hline Lead & $5.10 \mathrm{E} 0$ & N/A & $"$ & $5.00 \mathrm{E} 0$ & $7.45 \mathrm{E}-3$ & 102 & $75-125$ & \\
\hline
\end{tabular}


RCRA Metals By PNNL-AGG-415/1:5 Water Extract - Quality Control

Environmental Science Laboratory

\begin{tabular}{|lrrrrrrrrrrr}
\hline & & Reporting & & Spike & Source & & & $\%$ REC & & RPD & \\
Analyte & Result & Limit & Units & Level & Result & $\%$ REC & Limits & RPD & Limit & Notes \\
\hline
\end{tabular}

Batch 1A17003 - 1:5 Water Extract (ICP/ICPMS)

\begin{tabular}{|c|c|c|c|c|c|c|c|c|}
\hline \multirow{2}{*}{$\begin{array}{l}\text { Blank (1A17003-BLK1) } \\
\text { Chromium }\end{array}$} & \multicolumn{8}{|c|}{ Prepared \& Analyzed: 01/17/11 } \\
\hline & $<3.44 \mathrm{E}-3$ & $3.44 \mathrm{E}-3$ & ug/g wet & & & & & \\
\hline Arsenic & $<1.42 \mathrm{E}-2$ & $1.42 \mathrm{E}-2$ & $"$ & & & & & \\
\hline Selenium & $<7.92 \mathrm{E}-3$ & $7.92 \mathrm{E}-3$ & $"$ & & & & & \\
\hline Silver & $<3.13 \mathrm{E}-3$ & $3.13 \mathrm{E}-3$ & $"$ & & & & & \\
\hline Cadmium & $<5.73 \mathrm{E}-4$ & $5.73 \mathrm{E}-4$ & $"$ & & & & & \\
\hline Lead & $<1.16 \mathrm{E}-3$ & $1.16 \mathrm{E}-3$ & $"$ & & & & & \\
\hline LCS (1A17003-BS1) & \multicolumn{8}{|c|}{ Prepared \& Analyzed: 01/17/11 } \\
\hline Chromium & 4.77E0 & $3.44 \mathrm{E}-1$ & ug/g wet & $5.00 \mathrm{E} 0$ & 95.5 & $80-120$ & & \\
\hline Arsenic & $4.56 \mathrm{E} 0$ & $2.84 \mathrm{E}-1$ & $"$ & $5.00 \mathrm{E} 0$ & 91.2 & $80-120$ & & \\
\hline Selenium & $4.90 \mathrm{E} 0$ & $7.92 \mathrm{E}-1$ & $"$ & $5.00 \mathrm{E} 0$ & 98.1 & $80-120$ & & \\
\hline Silver & 4.65E0 & $3.13 \mathrm{E}-1$ & $"$ & $5.00 \mathrm{E} 0$ & 93.1 & $80-120$ & & \\
\hline Cadmium & 4.57E0 & $5.73 \mathrm{E}-2$ & $"$ & $5.00 \mathrm{E} 0$ & 91.5 & $80-120$ & & \\
\hline Lead & 4.64E0 & $1.16 \mathrm{E}-1$ & $"$ & $5.00 \mathrm{E} 0$ & 92.9 & $80-120$ & & \\
\hline Duplicate (1A17003-DUP1) & \multicolumn{3}{|c|}{ Source: $1009008-10$} & \multicolumn{5}{|c|}{ Prepared \& Analyzed: 01/17/11 } \\
\hline Chromium & $2.19 \mathrm{E}-2$ & $1.43 \mathrm{E}-2$ & ug/g dry & & & & 0.548 & 35 \\
\hline Arsenic & $1.35 \mathrm{E}-1$ & $5.90 \mathrm{E}-2$ & $"$ & & & & 20.3 & 35 \\
\hline Selenium & $<3.29 \mathrm{E}-2$ & $3.29 \mathrm{E}-2$ & $"$ & & & & & 35 \\
\hline Silver & $<1.30 \mathrm{E}-2$ & $1.30 \mathrm{E}-2$ & $"$ & & & & & 35 \\
\hline Cadmium & $<2.38 \mathrm{E}-3$ & $2.38 \mathrm{E}-3$ & $"$ & & & & & 35 \\
\hline Lead & $<4.83 \mathrm{E}-3$ & $4.83 \mathrm{E}-3$ & $"$ & & & & & 35 \\
\hline
\end{tabular}


RCRA Metals By PNNL-AGG-415/Acid Extract - Quality Control

Environmental Science Laboratory

\begin{tabular}{|lrrrrrrrrrrrr}
\hline & & Reporting & & Spike & Source & & & $\%$ REC & & RPD \\
Analyte & Result & Limit & Units & Level & Result & $\%$ REC & Limits & RPD & Limit & Notes \\
\hline
\end{tabular}

Batch 0J11001 - ASTM D 5198 (ICP/ICPMS)

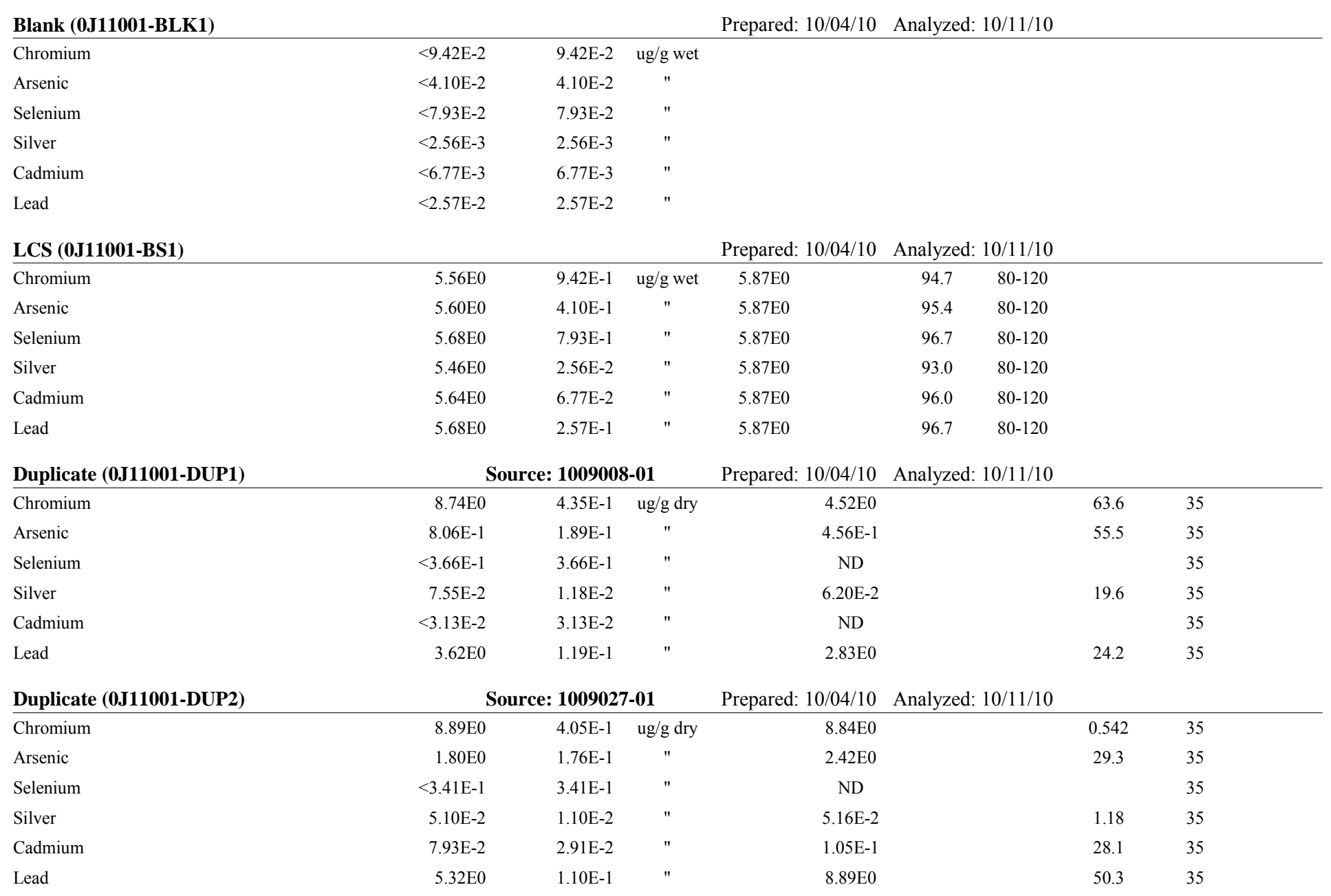


RCRA Metals By PNNL-AGG-415/Acid Extract - Quality Control

Environmental Science Laboratory

\begin{tabular}{|lrrrrrrrrrrrr}
\hline & & Reporting & & Spike & Source & & & $\%$ REC & & RPD \\
Analyte & Result & Limit & Units & Level & Result & $\%$ REC & Limits & RPD & Limit & Notes \\
\hline
\end{tabular}

Batch 0J11001 - ASTM D 5198 (ICP/ICPMS)

\begin{tabular}{llccccccc}
\hline Post Spike (0J11001-PS1) & \multicolumn{3}{c}{ Source: 1009008-06 } & \multicolumn{2}{c}{ Prepared \& Analyzed: $10 / 11 / 10$} \\
\hline Chromium & $3.28 \mathrm{E} 1$ & N/A & ug/L & $5.00 \mathrm{E} 0$ & $2.82 \mathrm{E} 1$ & 91.7 & $75-125$ \\
Arsenic & $8.74 \mathrm{E} 0$ & N/A & $"$ & $5.00 \mathrm{E} 0$ & $3.86 \mathrm{E} 0$ & 97.6 & $75-125$ \\
Selenium & $4.89 \mathrm{E} 0$ & N/A & $"$ & $5.00 \mathrm{E} 0$ & $7.07 \mathrm{E}-2$ & 96.3 & $75-125$ \\
Silver & $4.75 \mathrm{E} 0$ & N/A & $"$ & $5.00 \mathrm{E} 0$ & $4.98 \mathrm{E}-1$ & 85 & $75-125$ \\
Cadmium & $5.78 \mathrm{E} 0$ & N/A & $"$ & $5.00 \mathrm{E} 0$ & $1.21 \mathrm{E} 0$ & 91.4 & $75-125$ \\
Lead & $2.10 \mathrm{E} 1$ & N/A & " & $5.00 \mathrm{E} 0$ & $1.76 \mathrm{E} 1$ & 67.9 & $75-125$
\end{tabular}

Batch 1A20001 - ASTM D 5198 (ICP/ICPMS)

\begin{tabular}{|c|c|c|c|c|c|c|c|c|}
\hline \multirow{2}{*}{$\begin{array}{l}\text { Blank (1A20001-BLK1) } \\
\text { Chromium }\end{array}$} & \multicolumn{8}{|c|}{ Prepared \& Analyzed: 01/20/11 } \\
\hline & $<1.88 \mathrm{E}-1$ & $1.88 \mathrm{E}-1$ & ug/g wet & & & & & \\
\hline Arsenic & $<8.20 \mathrm{E}-2$ & $8.20 \mathrm{E}-2$ & $"$ & & & & & \\
\hline Selenium & $<1.59 \mathrm{E}-1$ & $1.59 \mathrm{E}-1$ & $"$ & & & & & \\
\hline Silver & $<5.13 \mathrm{E}-3$ & $5.13 \mathrm{E}-3$ & $"$ & & & & & \\
\hline Cadmium & $<1.35 \mathrm{E}-2$ & $1.35 \mathrm{E}-2$ & $"$ & & & & & \\
\hline Lead & $<5.13 \mathrm{E}-2$ & $5.13 \mathrm{E}-2$ & $"$ & & & & & \\
\hline LCS (1A20001-BS1) & \multicolumn{8}{|c|}{ Prepared \& Analyzed: 01/20/11 } \\
\hline Chromium & $5.30 \mathrm{E} 0$ & $9.42 \mathrm{E}-1$ & $\mathrm{ug} / \mathrm{g}$ wet & $5.80 \mathrm{E} 0$ & 91.5 & $80-120$ & & \\
\hline Arsenic & $5.84 \mathrm{E} 0$ & $4.10 \mathrm{E}-1$ & $"$ & $5.80 \mathrm{E} 0$ & 101 & $80-120$ & & \\
\hline Selenium & $5.29 \mathrm{E} 0$ & 7.93E-1 & $"$ & $5.80 \mathrm{E} 0$ & 91.3 & $80-120$ & & \\
\hline Silver & $5.40 \mathrm{E} 0$ & $2.56 \mathrm{E}-2$ & $"$ & $5.80 \mathrm{E} 0$ & 93.3 & $80-120$ & & \\
\hline Cadmium & $5.40 \mathrm{E} 0$ & $6.77 \mathrm{E}-2$ & $"$ & $5.80 \mathrm{E} 0$ & 93.3 & $80-120$ & & \\
\hline Lead & $5.52 \mathrm{E} 0$ & $2.57 \mathrm{E}-1$ & $"$ & $5.80 \mathrm{E} 0$ & 95.3 & $80-120$ & & \\
\hline Duplicate (1A20001-DUP1) & \multicolumn{3}{|c|}{ Source: 1009008-10 } & \multicolumn{5}{|c|}{ Prepared \& Analyzed: 01/20/11 } \\
\hline Chromium & $8.41 \mathrm{E} 0$ & $8.96 \mathrm{E}-1$ & ug/g dry & & & & 27.0 & 35 \\
\hline Arsenic & $8.27 \mathrm{E} 0$ & $3.90 \mathrm{E}-1$ & $"$ & & & & 15.9 & 35 \\
\hline Selenium & $<7.54 \mathrm{E}-1$ & $7.54 \mathrm{E}-1$ & $"$ & & & & & 35 \\
\hline Silver & $<2.44 \mathrm{E}-2$ & $2.44 \mathrm{E}-2$ & $"$ & & & & & 35 \\
\hline Cadmium & $<6.44 \mathrm{E}-2$ & $6.44 \mathrm{E}-2$ & $"$ & & & & & 35 \\
\hline Lead & $4.35 \mathrm{E} 0$ & $2.44 \mathrm{E}-1$ & $"$ & & & & 15.8 & 35 \\
\hline
\end{tabular}




\section{Strontium 90/Acid Extract - Quality Control}

Environmental Science Laboratory

\begin{tabular}{|lrrrrrrrrrrr}
\hline & & Reporting & & Spike & Source & & \%REC & & RPD \\
Analyte & Result & Limit & Units & Level & Result & \%REC & Limits & RPD & Limit & Notes \\
\hline
\end{tabular}

\section{Batch 0J07001 - ASTM D 5198 (RadChem)}

Blank (0J07001-BLK1)

Strontium-90

$<2.66 \mathrm{E}-1 \quad 2.66 \mathrm{E}-1 \quad \mathrm{pCi} / \mathrm{g}$ wet

Prepared: 10/07/10 Analyzed: 10/15/10

LCS (0J07001-BS1)

Strontium-90

Prepared: $10 / 07 / 10$ Analyzed: $10 / 15 / 10$

Duplicate (0J07001-DUP1)

Strontium-90

Source: 1009008-01

Prepared: 10/07/10 Analyzed: 10/15/10

Duplicate (0J07001-DUP2)

Strontium-90

Source: 1009027-01

ND

35

Strontium-90

$<9.04 \mathrm{E}-1 \quad 9.04 \mathrm{E}-1 \quad \mathrm{pCi} / \mathrm{g}$ dry

Prepared: 10/07/10 Analyzed: 10/15/10

Matrix Spike (0J07001-MS1)

Source: 1009008-01 Prepared: 10/07/10 Analyzed: 10/15/10

Strontium-90

$\begin{array}{lllllll}9.43 \mathrm{E} 1 & 2.66 \mathrm{E}-1 & \mathrm{pCi} / \mathrm{g} \text { dry } & 1.02 \mathrm{E} 2 & \mathrm{ND} & 97.5 & 75-125\end{array}$

Batch 1A05001 - ASTM D 5198 (RadChem)

Blank (1A05001-BLK1)

Prepared: 01/05/11 Analyzed: 01/12/11

Strontium-90

$<3.21 \mathrm{E}-1 \quad 3.21 \mathrm{E}-1 \quad \mathrm{pCi} / \mathrm{g}$ wet

LCS (1A05001-BS1)

Prepared: 01/05/11 Analyzed: 01/12/11

Strontium-90

$8.97 \mathrm{E} 1$

3.21E-1 pCi/g wet $9.63 \mathrm{E} 1$

$93.1 \quad 80-120$

Duplicate (1A05001-DUP1)

Source: 1009008-10 Prepared: 01/05/11 Analyzed: 01/12/11

Strontium-90

$1.10 \mathrm{E} 0 \quad \mathrm{pCi} / \mathrm{g}$ dry

ND

35

Matrix Spike (1A05001-MS1)

Strontium-90

\begin{tabular}{cccccc} 
Source: $\mathbf{1 0 0 9 0 0 8 - 1 0}$ & \multicolumn{2}{c}{ Prepared: $01 / 05 / 11$} & Analyzed: 01/12/11 \\
\hline $3.21 \mathrm{E}-1$ & $\mathrm{pCi} / \mathrm{g}$ dry & $2.05 \mathrm{E} 2$ & $\mathrm{ND}$ & 93.6 & $75-125$
\end{tabular} 


\section{Strontium 90/1:1 Water Extract - Quality Control}

Environmental Science Laboratory

\begin{tabular}{|lrrrrrrrrrrr}
\hline & & Reporting & & Spike & Source & & & $\%$ REC & & RPD & \\
Analyte & Result & Limit & Units & Level & Result & $\%$ REC & Limits & RPD & Limit & Notes \\
\hline
\end{tabular}

\section{Batch 0J07010 - 1:1 Water Extract (RadChem)}

Blank (0J07010-BLK1)

Strontium-90

$<9.31 \mathrm{E}-1 \quad 9.31 \mathrm{E}-1 \quad \mathrm{pCi} / \mathrm{g}$ wet

Prepared: 10/07/10 Analyzed: 10/15/10

LCS (0J07010-BS1)

Strontium-90

$9.52 \mathrm{E} 1$

Prepared: 10/07/10 Analyzed: 10/15/10

Duplicate (0J07010-DUP1)

Strontium-90

Source: 1009008-01

Prepared: 10/07/10 Analyzed: 10/15/10

$<8.70 \mathrm{E}-1 \quad 8.70 \mathrm{E}-1 \quad \mathrm{pCi} / \mathrm{g}$ dry

ND

35

Matrix Spike (0J07010-MS1)

Strontium-90

Source: 1009008-01

Prepared: 10/07/10 Analyzed: 10/15/10

Batch 1A06003 - 1:1 Water Extract (RadChem)

Blank (1A06003-BLK1)

Prepared: 01/06/11 Analyzed: 01/12/11

Strontium-90

$<1.12 \mathrm{E} 0$

$1.12 \mathrm{E} 0 \quad \mathrm{pCi} / \mathrm{g}$ wet

LCS (1A06003-BS1)

Prepared: 01/06/11 Analyzed: 01/12/11

Strontium-90

$9.41 \mathrm{E} 1$

$1.12 \mathrm{E} 0 \quad \mathrm{pCi} / \mathrm{g}$ wet $9.63 \mathrm{E} 1$

$80-120$

Duplicate (1A06003-DUP1)

Source: 1009008-10 Prepared: 01/06/11 Analyzed: 01/12/11

Strontium-90

$<1.30 \mathrm{E} 0 \quad 1.30 \mathrm{E} 0 \quad \mathrm{pCi} / \mathrm{g}$ dry

ND

35

Matrix Spike (1A06003-MS1)

Strontium-90

\begin{tabular}{cccccc} 
9.36E1 & Prepared: 01/06/11 & Analyzed: 01/12/11 \\
\hline
\end{tabular} 
Strontium 90/1:2.5 Water Extract - Quality Control

Environmental Science Laboratory

\begin{tabular}{|lrrrrrrrrrrrr}
\hline & & Reporting & & Spike & Source & & & $\%$ REC & & RPD \\
Analyte & Result & Limit & Units & Level & Result & $\%$ REC & Limits & RPD & Limit & Notes \\
\hline
\end{tabular}

Batch 0J07011 - 1:2.5 Water Extract (RadChem)

Blank (0J07011-BLK1)

Strontium-90

LCS (0J07011-BS1)

Strontium-90

Duplicate (0J07011-DUP1)

Strontium-90

Matrix Spike (0J07011-MS1)

Strontium-90

Batch 1A07003 - 1:2.5 Water Extract (RadChem)

Blank (1A07003-BLK1)

Strontium-90

$<1.11 \mathrm{E} 0$

$1.11 \mathrm{E} 0 \quad \mathrm{pCi} / \mathrm{g}$ wet

LCS (1A07003-BS1)

Strontium-90

$9.40 \mathrm{E} 1$

Duplicate (1A07003-DUP1)

Strontium-90

$<2.76 \mathrm{E}$

Matrix Spike (1A07003-MS1)

Strontium-90

9.31E-1 pCi/g wet
Prepared \& Analyzed: 11/15/10

Prepared \& Analyzed: 11/15/10

9.31E-1 pCi/g wet $\quad 9.67 \mathrm{E} 1 \quad 96.9 \quad 80-120$

Source: 1009008-01 Prepared \& Analyzed: 11/15/10

$2.23 \mathrm{E} 0 \mathrm{pCi} / \mathrm{g}$ dry ND

35

Source: 1009008-01 Prepared \& Analyzed: 11/15/10

$\begin{array}{llllll}1.58 \mathrm{E} 0 & \mathrm{pCi} / \mathrm{g} \text { dry } & 1.02 \mathrm{E} 2 & \mathrm{ND} & 99.2 & 75-125\end{array}$

Prepared: 01/07/11 Analyzed: 01/17/11

Prepared: 01/07/11 Analyzed: 01/17/11

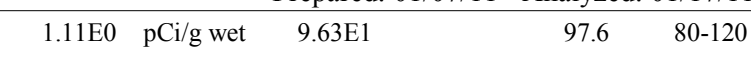

Source: 1009008-10 Prepared: 01/07/11 Analyzed: 01/17/11

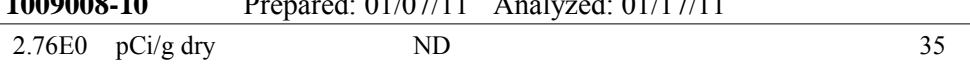

\begin{tabular}{ccccccc}
\multicolumn{2}{c}{ Source: 1009008-10 } & \multicolumn{2}{c}{ Prepared: $01 / 07 / 11$} & Analyzed: 01/17/11 \\
\hline $9.30 \mathrm{E} 1$ & $1.95 \mathrm{E} 0$ & pCi/g dry & $1.02 \mathrm{E} 2$ & $7.11 \mathrm{E}-1$ & 90.2 & $75-125$
\end{tabular} 


\section{Strontium 90/1:5 Water Extract - Quality Control}

Environmental Science Laboratory

\begin{tabular}{|lrrrrrrrrrrr}
\hline & & Reporting & & Spike & Source & & \%REC & & RPD \\
Analyte & Result & Limit & Units & Level & Result & $\%$ REC & Limits & RPD & Limit & Notes \\
\hline
\end{tabular}

\section{Batch 0J07012 - 1:5 Water Extract (RadChem)}

Blank (0J07012-BLK1)

Strontium-90

$<9.31 \mathrm{E}-1 \quad 9.31 \mathrm{E}-1 \quad \mathrm{pCi} / \mathrm{g}$ wet

Prepared: 10/07/10 Analyzed: 10/15/10

LCS (0J07012-BS1)

Strontium-90

$9.53 \mathrm{E} 1$

Prepared: 10/07/10 Analyzed: 10/15/10

Duplicate (0J07012-DUP1)

Strontium-90

Source: 1009008-01

Prepared: 10/07/10 Analyzed: 10/15/10

$<4.43 \mathrm{E} 0 \quad 4.43 \mathrm{E} 0 \quad \mathrm{pCi} / \mathrm{g}$ dry

ND

35

Matrix Spike (0J07012-MS1)

Source: 1009008-01 Prepared: 10/07/10 Analyzed: 10/15/10

Strontium-90

$\begin{array}{lllllll}9.88 \mathrm{E} 1 & 1.58 \mathrm{E} 0 & \mathrm{pCi} / \mathrm{g} \text { dry } & 1.02 \mathrm{E} 2 & \mathrm{ND} & 101 & 75-125\end{array}$

Batch 1A10006 - 1:5 Water Extract (RadChem)

Blank (1A10006-BLK1)

Prepared: 01/10/11 Analyzed: 01/17/11

Strontium-90

$<1.11 \mathrm{E} 0 \quad 1.11 \mathrm{E} 0 \quad \mathrm{pCi} / \mathrm{g}$ wet

LCS (1A10006-BS1)

Prepared: 01/10/11 Analyzed: 01/17/11

Strontium-90

9.40E1

$1.11 \mathrm{E} 0 \quad \mathrm{pCi} / \mathrm{g}$ wet $9.63 \mathrm{E} 1$

$80-120$

Duplicate (1A10006-DUP1)

Source: 1009008-10 Prepared: 01/10/11 Analyzed: 01/17/11

Strontium-90

$<4.61 \mathrm{E} 0 \quad 4.61 \mathrm{E} 0 \quad \mathrm{pCi} / \mathrm{g}$ dry

ND

35

Matrix Spike (1A10006-MS1)

Strontium-90

\begin{tabular}{lccccc} 
9.17E1 & Prepared: 01/10/11 & Analyzed: $01 / 17 / 11$ \\
\hline
\end{tabular}




\begin{tabular}{|c|c|c|}
\hline \multicolumn{3}{|c|}{ Strontium 90 - Quality Control } \\
\hline \multicolumn{3}{|c|}{ Batch 0J07001- ASTM D 5198 (RadChem) } \\
\hline Sample ID & HEIS ID & Tracer Recovery \\
\hline $1009008-01$ & B274K7 & $94.9 \%$ \\
\hline 1009008-02 & B273B8 & $94.3 \%$ \\
\hline 1009008-03 & B27PY0 & $94.2 \%$ \\
\hline 1009008-04 & $\mathrm{B} 274 \mathrm{~K} 6$ & $92.7 \%$ \\
\hline 1009008-05 & B26TC1 & $92.7 \%$ \\
\hline $1009008-06$ & B26TC2 & $95.5 \%$ \\
\hline 1009008-07 & B273B2 & $95.2 \%$ \\
\hline 1009008-08 & B273B3 & $93.8 \%$ \\
\hline 1009008-09 & B273B4 & $93.6 \%$ \\
\hline $1009008-10$ & B273B6 & $94.6 \%$ \\
\hline $1009008-11$ & B273B5 & $93.4 \%$ \\
\hline \multicolumn{3}{|c|}{ Strontium 90 - Quality Control } \\
\hline \multicolumn{3}{|c|}{ Batch 0J07010 - 1:1 Water Extract (RadChem) } \\
\hline Sample ID & HEIS ID & Tracer Recovery \\
\hline $1009008-01$ & $\mathrm{~B} 274 \mathrm{~K} 7$ & $93.0 \%$ \\
\hline 1009008-02 & B273B8 & $93.3 \%$ \\
\hline 1009008-03 & B27PY0 & $91.7 \%$ \\
\hline 1009008-04 & B274K6 & $91.6 \%$ \\
\hline $1009008-05$ & B26TC1 & $92.4 \%$ \\
\hline 1009008-06 & B26TC2 & $92.8 \%$ \\
\hline 1009008-07 & B273B2 & $90.7 \%$ \\
\hline $1009008-08$ & B273B3 & $91.5 \%$ \\
\hline 1009008-09 & B273B4 & $91.0 \%$ \\
\hline $1009008-10$ & B273B6 & $92.0 \%$ \\
\hline 1009008-11 & B273B5 & $91.0 \%$ \\
\hline \multicolumn{3}{|c|}{ Strontium 90 - Quality Control } \\
\hline \multicolumn{3}{|c|}{ Batch 0J07011 - 1:2.5 Water Extract (RadChem) } \\
\hline Sample ID & HEIS ID & Tracer Recovery \\
\hline 1009008-01 & B274K7 & $89.4 \%$ \\
\hline 1009008-02 & B273B8 & $91.0 \%$ \\
\hline 1009008-03 & B27PY0 & $90.6 \%$ \\
\hline $1009008-04$ & B274K6 & $92.3 \%$ \\
\hline $1009008-05$ & B26TC1 & $90.8 \%$ \\
\hline $1009008-06$ & B26TC2 & $90.6 \%$ \\
\hline $1009008-07$ & B273B2 & $91.1 \%$ \\
\hline 1009008-08 & B273B3 & $90.9 \%$ \\
\hline 1009008-09 & B273B4 & $92.4 \%$ \\
\hline $1009008-10$ & B273B6 & $90.0 \%$ \\
\hline $1009008-11$ & B273B5 & $90.5 \%$ \\
\hline \multicolumn{3}{|c|}{ Strontium 90 - Quality Control } \\
\hline \multicolumn{3}{|c|}{ Batch 0J07012 - 1:5 Water Extract (RadChem) } \\
\hline Sample ID & HEIS ID & Tracer Recovery \\
\hline 1009008-01 & B274K7 & $90.7 \%$ \\
\hline 1009008-02 & B273B8 & $91.4 \%$ \\
\hline $1009008-03$ & B27PY0 & $91.3 \%$ \\
\hline $1009008-04$ & B274K6 & $91.2 \%$ \\
\hline $1009008-05$ & B26TC1 & $91.5 \%$ \\
\hline $1009008-06$ & B26TC2 & $91.9 \%$ \\
\hline 1009008-07 & B273B2 & $89.6 \%$ \\
\hline $1009008-08$ & B273B3 & $91.6 \%$ \\
\hline 1009008-09 & B273B4 & $90.4 \%$ \\
\hline $1009008-10$ & B273B6 & $92.5 \%$ \\
\hline $1009008-11$ & B273B5 & $90.2 \%$ \\
\hline
\end{tabular}




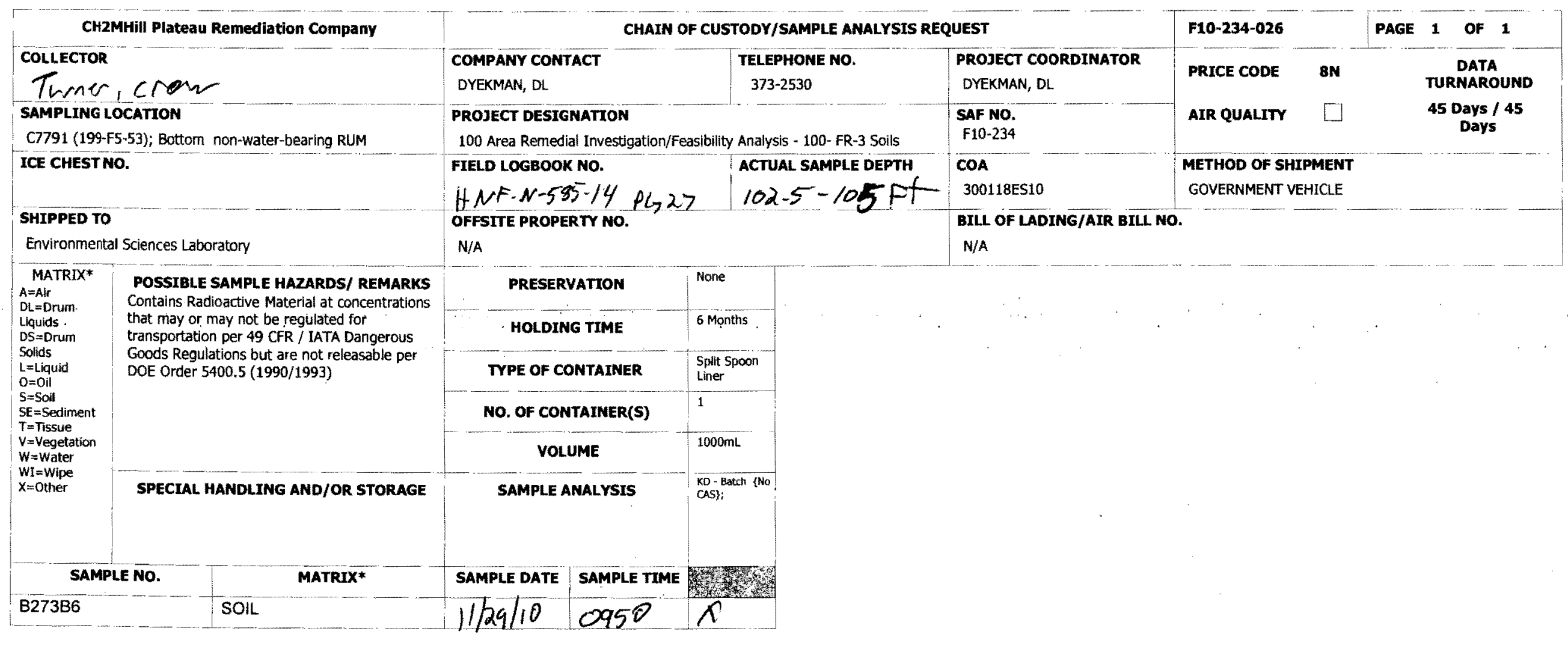

\begin{tabular}{|c|c|c|c|}
\hline \multicolumn{2}{|c|}{ CHAIN OF POSSESSION } & SIGN/ PRINT NAMES & \multirow{2}{*}{$\begin{array}{l}\text { SPECIAL INSTRUCTIONS } \\
\text { ** The } 100 \text { Area S\&GRP Characterization and Monitoring Sampling and } \\
\text { Analysis GKI applies to this SAF. } \\
\qquad B R M \# 13564\end{array}$} \\
\hline \multicolumn{2}{|c|}{ 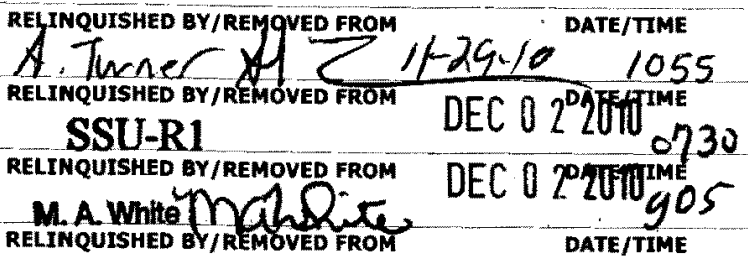 } & 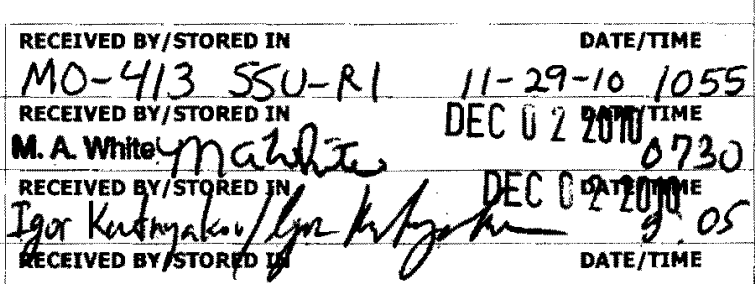 & \\
\hline \multicolumn{2}{|c|}{ RELINQUISHED BY/REMOVED FROM } & RECEIVED BY/STORED IN & \\
\hline \multicolumn{2}{|c|}{ RELINQUISHED BY/REMOVED FROM } & RECEIVED BY/STORED IN & \\
\hline \multicolumn{2}{|c|}{ RELINQQUISHED BY/REMOVED FROM } & RECEIVED BY/STORED IN & \\
\hline $\begin{array}{l}\text { LABORATORY } \\
\text { SECTION }\end{array}$ & RECEIVED BY & & DATE/TMME \\
\hline $\begin{array}{l}\text { FINAL SAMPLE } \\
\text { DISPOSITION }\end{array}$ & DISPOSAL METHOD & & DISPOSED BY \\
\hline
\end{tabular}




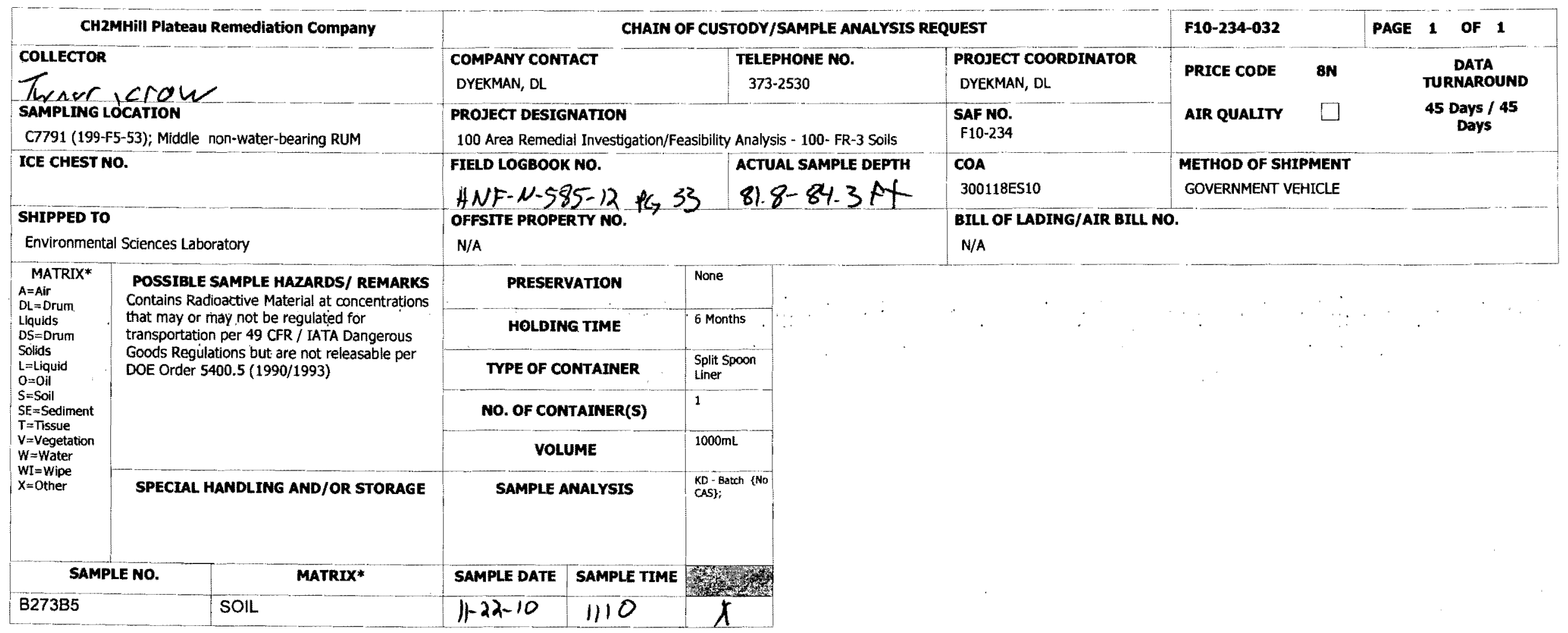

\section{CHAIN OF POSSESSION}

REYNQUISHED BY/REMOVED FROM 4. Twner LL RELINQUUSHED BY/RETOVED FROM SSI-R M.A. Whito UY/REMOVER FROM Nolitét RELINQUISHED BY/REMOVED FROM RELIMQUISHED BY/REMOVED FROM

RELIMQUISHED BY/REMOVED FROM —DATE/TIME

\begin{tabular}{|c|l|}
\hline $\begin{array}{c}\text { LABORATORY } \\
\text { SECTION }\end{array}$ & RECETVED BY \\
\hline $\begin{array}{c}\text { FINAL SAMPLE } \\
\text { DISPOSIMION }\end{array}$ & DISPOSAL METHOD \\
\hline
\end{tabular}

\section{SIGN/ PRINT NAMES}

\section{RECEIVED BY/STORED IN}

RECEIVE BY/STORED IN $-4 / 35 S U-R / 11-22-10 / 215$ DEC 0220 fị̂ Ohe/TME $^{\mathrm{T}}$

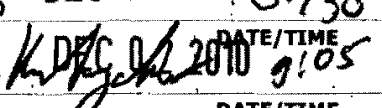
RECEIVED QYis Foped IP

DATE/TIME DATE/TIME DATE/TIME

RECEIVED BY/STORED IN RECEIVED BY/STORED IN

DATE/TIME DATE/TIME RECEIVED BY/STORED IN DATE/TIME

\section{SPECIAL INSTRUCTIONS}

** The 100 Area S\&GRP Characterization and Monitoring Sampling and Analysis GKI applies to this SAF.

$$
\text { BRM\# } 13564
$$

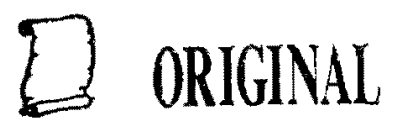




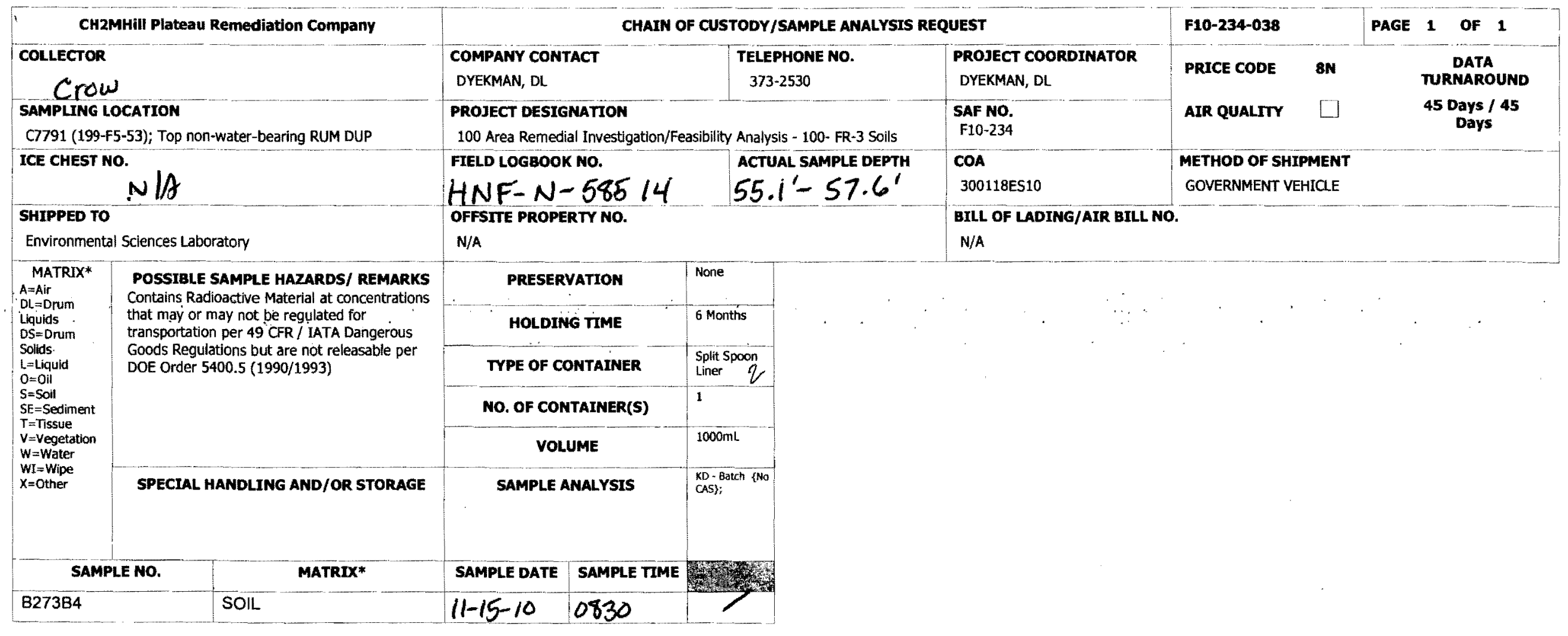

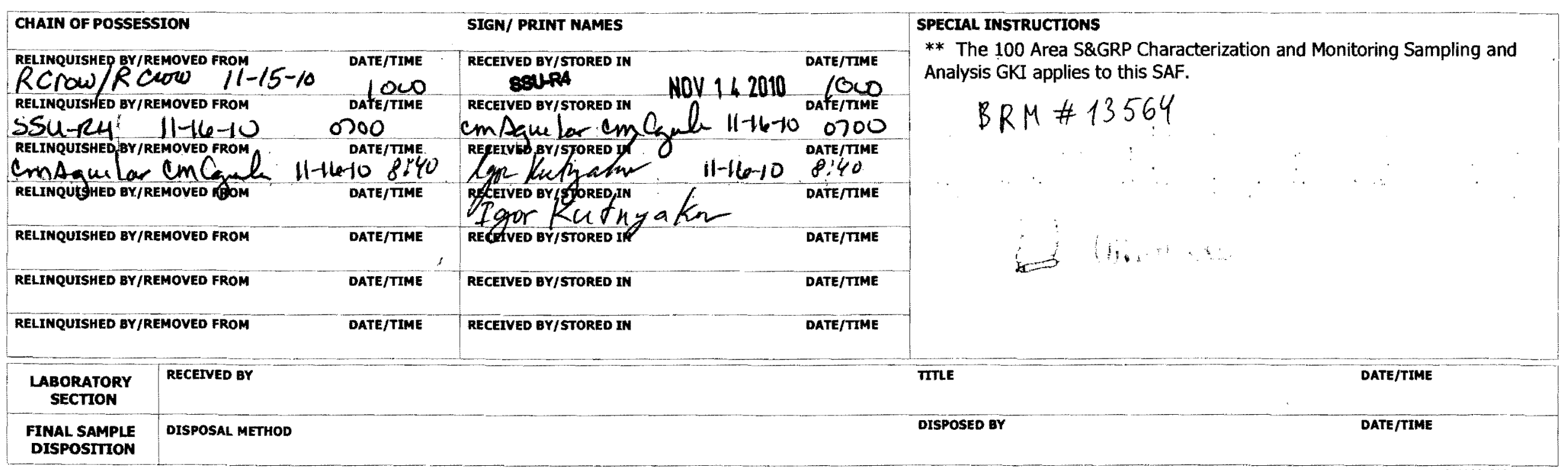




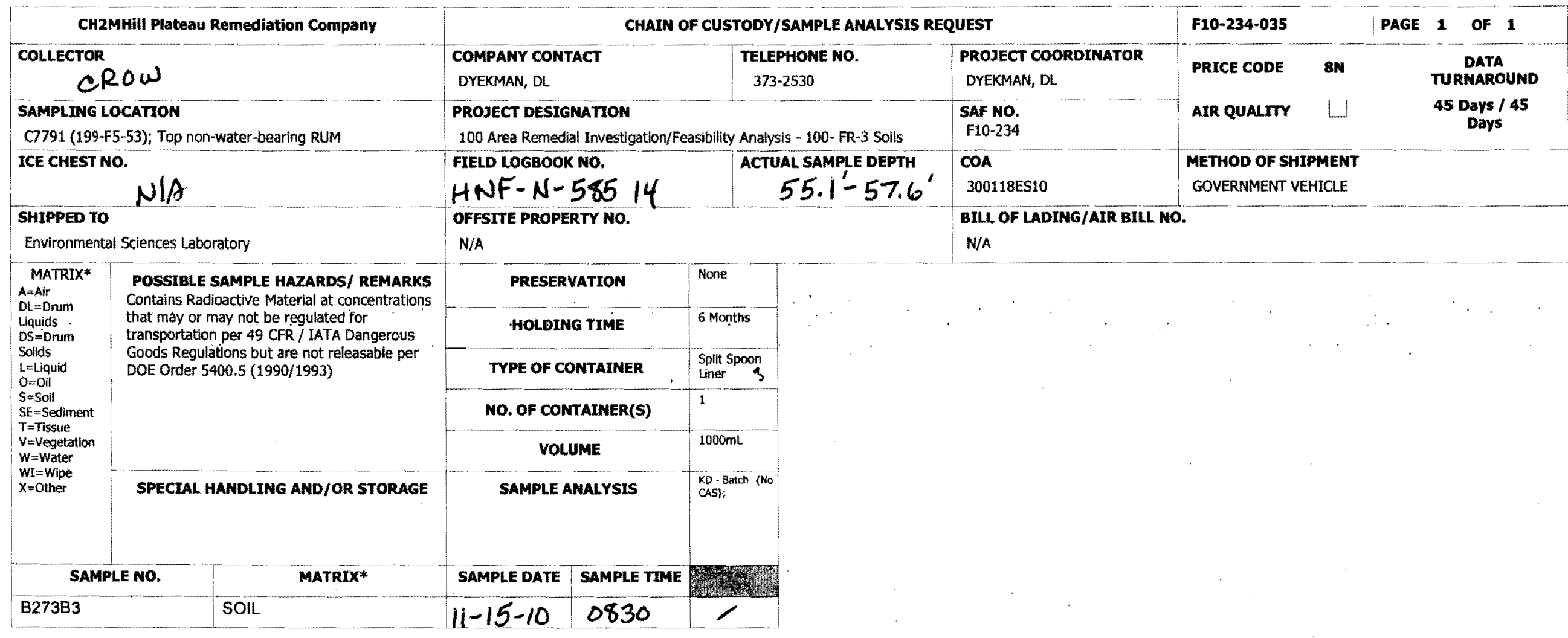

\begin{tabular}{|c|c|c|c|c|}
\hline \multicolumn{3}{|c|}{ CHAIN OF POSSESSION } & SIGN/ PRINT NAMES & \multirow{2}{*}{$\begin{array}{l}\text { SPECIAL INSTRUCTIONS } \\
\text { ** The } 100 \text { Area S\&GRP Characterization and Monitoring Sampling and } \\
\text { Analysis GKI applies to this SAF. }\end{array}$} \\
\hline \multicolumn{2}{|c|}{ RELINQUISHED,BY/REMOVED FROM } & $\begin{array}{l}\text { DATE/TIME } \\
1000\end{array}$ & $\begin{array}{c}\text { RECEIVED BY/STORED IN } \\
\text { ESUR4 }\end{array}$ & \\
\hline \multirow{2}{*}{\multicolumn{2}{|c|}{$\begin{array}{l}\text { RELINQUISHED BY/REMOVED FROM } \\
55 U-R 4\end{array}$}} & DATE/TIME & RECEIVED BY/STORED IN & \\
\hline & & 0700. & inAavilarcm $C_{a}$ h 11-1610:0700 & 110 \\
\hline \multicolumn{2}{|c|}{ RELINQUISHED BY/REMOVEP FROM , $11-16$} & $\begin{array}{l}\text { DATE } / \text { TIME } \\
-10,8,40\end{array}$ & Regived by / STORED IN & $\cdots \vdots \cdots$ \\
\hline \multicolumn{2}{|c|}{ CM Uquilar CMUlag } & DATE/TIME & REgEIVED BY/STqREg IN $\quad$ DATE/TIME & ' \\
\hline \multicolumn{2}{|c|}{ RELUNQUISHED BY/REMOVED FROM } & DATE/TIME & REGEIVED BY/STORED IN $\theta$ & 9 \\
\hline \multicolumn{2}{|c|}{ RELINQUISHED BY/REMOVED FROM } & DATE/TIME & RECEIVEO BY/STORED IN & $-\infty+1,14$ \\
\hline \multicolumn{2}{|c|}{ RELIMQUISHED BY/REMOVED FROM } & DATE/TIME & RECEIVED BY/STORED IM & \\
\hline $\begin{array}{l}\text { LABORATORY } \\
\text { SECTION }\end{array}$ & RECEIVED BY & & & DATE/TIME \\
\hline $\begin{array}{l}\text { FINAL SAMPLE } \\
\text { DISPOSTITON }\end{array}$ & DISPOSAL METHOD & & & DISPOSED BY \\
\hline
\end{tabular}


CH2MHill Plateau Remediation Company

\section{COLLECTOR}

\section{Twene con}

SAMPLING LOCATION

C7791 (199-F5-53); Bottom unconfined aquife

ICE CHEST NO.

$$
N \mid A
$$

\section{SHIPPED TO}

Environmental Sciences Laboratory

MATRIX* POSSIBLE SAMPLE HAZARDS/ REMARKS

A=Air

$\mathrm{DL}=\mathrm{Drum}$

Liquids

DS $=$ Dn

Solids

$L=L$ lquid
$0=0$ il

$\mathrm{S}=\mathrm{Sol}$

SE=Sediment

$T=$ Tissue

$V=$ vegetation

W=Water

WI $=$ Wipe
$X=$ Other

Contains Radioactive Material at concentration

that may. or may not be regulated for

transportation per 49 CFR / IATA Dangerous

(DOE Org 5400.5 (19 are not releasable per

DOE Order 5400.5 (1990/1993)

(n)

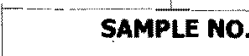

$\mathrm{B} 273 \mathrm{B2}$

SPECIAL HANDLING AND/OR STORAGE
CHAIN OF POSSESSION

RELINQUISHED BY/REMPVEO EROM

A. Twa $x$ RELINQUISHED BY/REMOVED FROM

SSU-R4: $\quad 11-14-10$

CmAvilar Am C RELINQUTSHED BYIREMOVED fROM

RELINQUISHED BY/REMOVED FROM

RELINQUISHED BY/REMOVED FROM

RELINQUISHED BY/REMOVED FROM
CHAIN OF CUSTODY/SAMPLE ANALYSIS REOUEST

COMPANY CONTACT

DYEKMAN, DL

PROJECT DESIGNATION

100 Area Remedial Investigation/Feasibility Analysis - 100- FR-3 Soils

FIELD LOGBOOK NO.

HNF-N-585-14 PG25 $52.3^{\prime}$ OFFSITE PROPERTY NO.

N/A

\begin{tabular}{|c|c|c|}
\hline \multicolumn{2}{|c|}{ PRESERVATION } & Nane \\
\hline \multicolumn{2}{|c|}{ HOLDING NIME } & 6 Months \\
\hline \multicolumn{2}{|c|}{ TYPE OF CONTAINER } & $\begin{array}{l}\text { Split Spoon } \\
\text { Liner }\end{array}$ \\
\hline \multicolumn{2}{|c|}{ NO. OF CONTAINER(S) } & 1 \\
\hline \multicolumn{2}{|c|}{ VOLUME } & $1000 \mathrm{~mL}$ \\
\hline \multicolumn{2}{|c|}{ SAMPLE ANALYSIS } & $\begin{array}{l}\text { KD- Batch } \\
\text { CASl; }\end{array}$ \\
\hline SAMPLE DATE & SAMPLE TIME & rese \\
\hline $11-11-10$ & 0850 & \\
\hline
\end{tabular}

N/A
F10-234-029

PAGE 1 OF 1

PRICE CODE DYEKMAN, DL

SAF NO.

F10-234

$\mathrm{COA}$

300118ES10

AIR QUALTTY

TURNAROUND

45 Days / 45

Days

METHOD OF SHIPMENT

GOVERNMENT VEHICLE
BILL OF LADING/AIR BILL NO

\section{B十\#}




\begin{tabular}{|c|c|c|c|c|c|c|c|c|c|}
\hline & Hill Plat & Kemediation Company & & CHAIN C & IF CUSTODY/S & QUEST & F10-234-042 & & PAGE 1 OF 1 \\
\hline COLLETO & ier & wherland & $\begin{array}{l}\text { COMPANY CONT } \\
\text { DYEKMAN, DL }\end{array}$ & & $\begin{array}{l}\text { TELEP } \\
373-2\end{array}$ & $\begin{array}{l}\text { PROJECT COORDINATOR } \\
\text { DYEKMAN, DL }\end{array}$ & PRICE CODE & & $\begin{array}{l}\text { DATA } \\
\text { TURNAROUND } \\
\text { 45 Dass / } 45\end{array}$ \\
\hline $\begin{array}{l}\text { SAMPLING } \\
\text { C7792 (199) }\end{array}$ & $\begin{array}{l}\text { CATriof } \\
-54) ; 5 f\end{array}$ & o RUM & $\begin{array}{l}\text { PROJECT DESIG } \\
100 \text { Area Remedi }\end{array}$ & $\begin{array}{l}\text { VATION } \\
\text { I Investigation/Fe }\end{array}$ & asibility Analysi & $\begin{array}{l}\text { SAF NO. } \\
\text { F10-234 }\end{array}$ & AIR QUALITY & $\square$ & \\
\hline ICE CHEST & & & $\begin{array}{l}\text { FIELD LOGBOOI } \\
\text { HNF-N - }\end{array}$ & No. & \begin{tabular}{l|l} 
ACTU \\
19 & 74.
\end{tabular} & $\begin{array}{l}\text { COA } \\
300118 E 510\end{array}$ & $\begin{array}{l}\text { METHOD OF SHI } \\
\text { GOVERNMENT VE }\end{array}$ & $\begin{array}{l}\text { PMENT } \\
\text { HICLE }\end{array}$ & \\
\hline $\begin{array}{l}\text { SHIPPED T } \\
\text { Environmen }\end{array}$ & Sciences & atory & $\begin{array}{l}\text { OFFSTTE PROPE } \\
\text { N/A }\end{array}$ & TY NO. & & $\begin{array}{l}\text { BILL Of LADING/ATR BTLLN } \\
\text { N/A }\end{array}$ & & & \\
\hline & POSSI: & SAMPLE HAZARDS/ REMARKS & PRESER & ATION & None & & & & \\
\hline $\begin{array}{l}\text { Liquids. } \\
\text { DS=Drum }\end{array}$ & $\begin{array}{l}\text { that ma } \\
\text { transpo }\end{array}$ & $\begin{array}{l}\text { ay not be regulated for } \\
\text { per } 49 \text { CFR / IATA Dangerous }\end{array}$ & HOLDIM & TIME & 6 Monthis & & & & \\
\hline 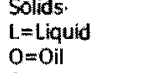 & & & TYPE OF C & NTAimer & $\begin{array}{l}\text { Split Spoon } \\
\text { iner }\end{array}$ & & & & \\
\hline 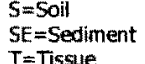 & & & NO. of con & TAIMER(S) & 1 & & & & \\
\hline 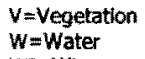 & & & vot & & 1000mL & & & & \\
\hline & SPEC & ANDLING AND/OR STORAGE & SAMPLE & NaLYsis & 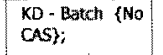 & & & & \\
\hline & E No. & MATRIX* & SAMPLE DATE & SAMPLE TMME & 等 & & & & \\
\hline B274K7 & & SOHL & $9-1-10$ & 1026 & $X$ & & & & \\
\hline
\end{tabular}

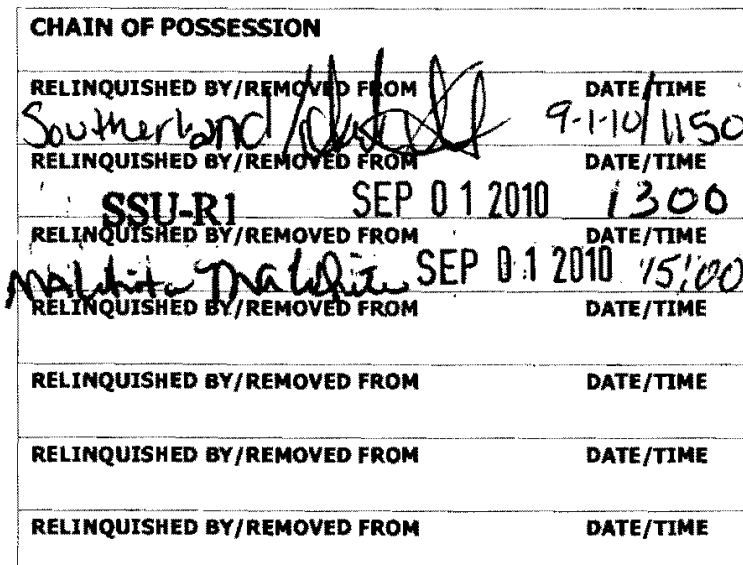

\section{SIGN/ PRINT NAMES} RECEUED BY/STORED IN 93 SSURI $9-1-10 / 1150$ RECEIVED BY/STORED IN

Metwhite M

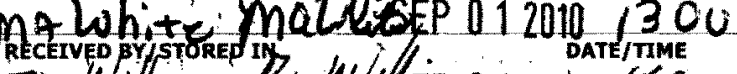
Be Di DatrTME RECEIVED BY/STORED IM RECEIVED BY/STORED IN DATE/TMME DATE/TTME

RECEIVED BY/STORED IM RECEIVED BY/STORED IN
DATE/TIME DATE/TIME

\section{SPECIAL INSTRUCTIONS}

** The CACN for all analytical work at WSCF laboratory is

401922ES20. ${ }^{* * *}$ The 100 Area S\&GRP Characterization and Monitoring Sampling and Analysis GKI applies to this SAF.

BRM \# 13564

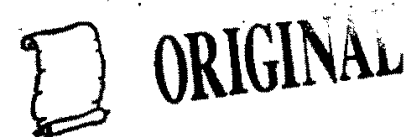

\begin{tabular}{|l|l|}
\hline $\begin{array}{c}\text { LABORATORY } \\
\text { SECTION }\end{array}$ & RECEIVED BY \\
\hline $\begin{array}{c}\text { FINAL SAMPLE } \\
\text { DISPOSITION }\end{array}$ & DISPOSAL METHOD \\
\hline
\end{tabular}




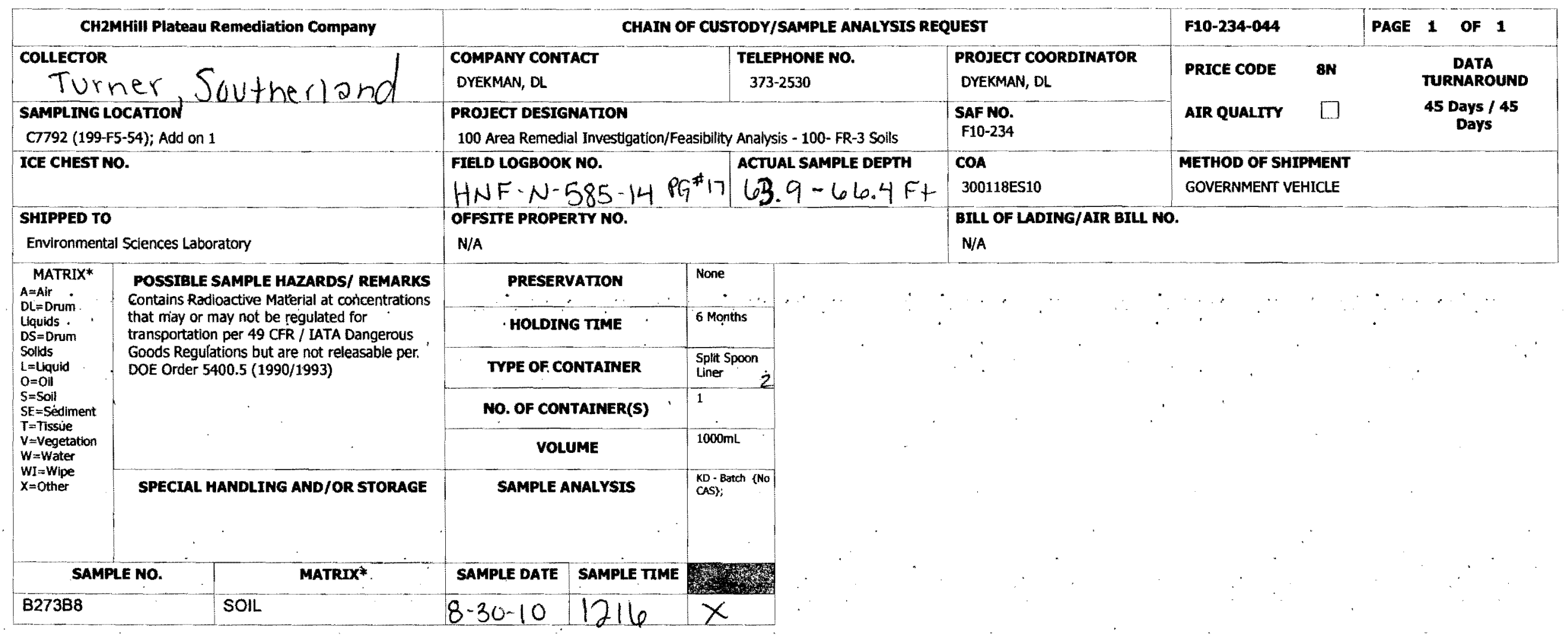

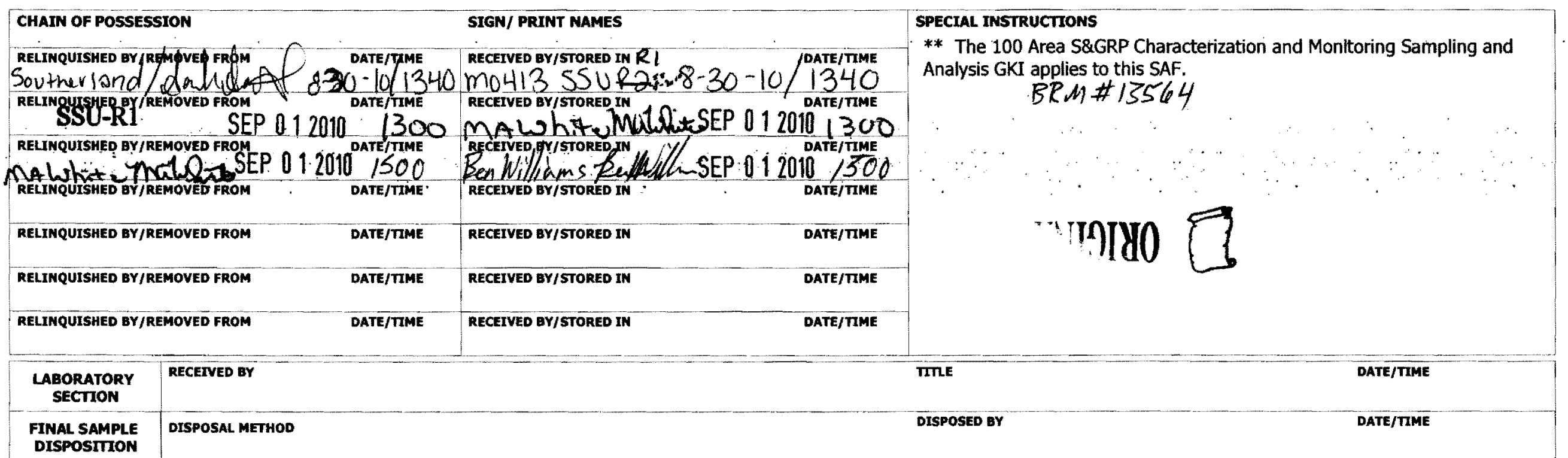


CH2MHill Plateau Remediation Company COLLECTOR

Turner southurmad SAMPLING LOCATION

C7792 (199-F5-54); Add an 2 ICE CHEST NO.

\section{SHIPPED TO}

Environmental Sciences Laboratory

MATRIX* POSSIBLE SAMPLE HAZARDS/ REMARKS

$A=$ Air

$\mathrm{DL}=\mathrm{Drum}$

Liquids

DS=Drum

Solids

$L=$ Liquid
$0=$ Oil

$\mathrm{S}=$ Soil

$\mathrm{S}=$ Soll
$\mathrm{SE}=$ Sediment

$T=$ rissue

$V=$ Vegetation

$W=$ Water

WI $=$ Wipe

Contains Radloactive Material at concentration that may or may not be regulated for transportation per 49 CFR / IATA Dangerous Goods Regultions but are not ra Das DOE Order 5400.5 (1990/1993)

$x=0$

$$
\text { SAMPLE NO }
$$

SPECIAL. HANDLING AND/OR STORAGE

B27PYO

SOIL

MATRIX*

MATRIX:

\section{CHAIN OF CUSTODY/SAMPLE ANALYSIS REQUEST}

\section{COMPANY CONTACT}

DYEKMAN, DL

\section{TELEPHONE NO.}

$373-2530$

\section{PROJECT DESIGNATION}

100 Area Remedial Investigation/Feasibility Analysis - 100- FR-3 Soils FIELD LOGBOOK NO.

ACTUAL SAMPLE DEPTH

WNF-N.585-14PG*1867.0-69.5 Ft OFFSITE PROPERTY NO.

N/A

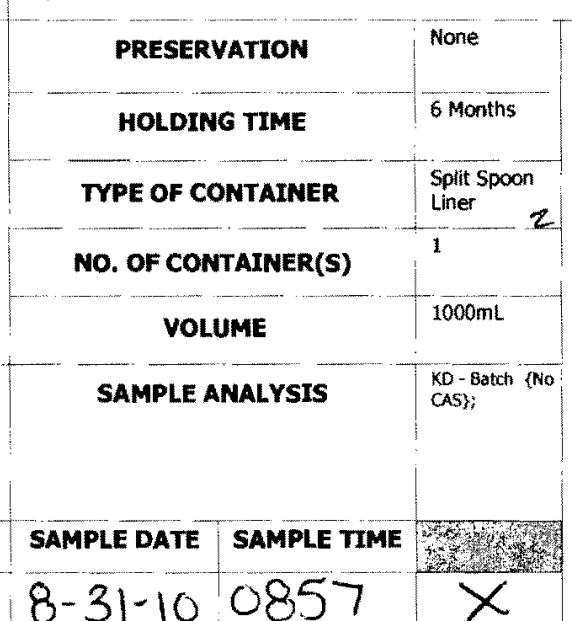

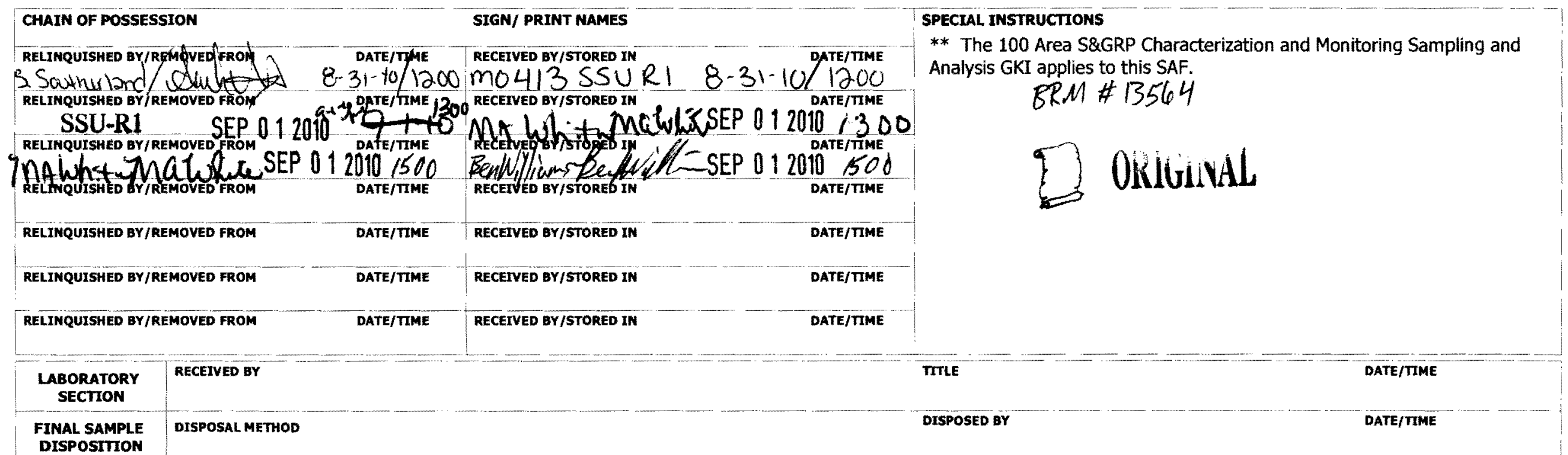

\section{CHAIN OF POSSESSION}

SELINQUISHED BY/REMQVED FROI

Southuiand Qunte

SSU-RI 


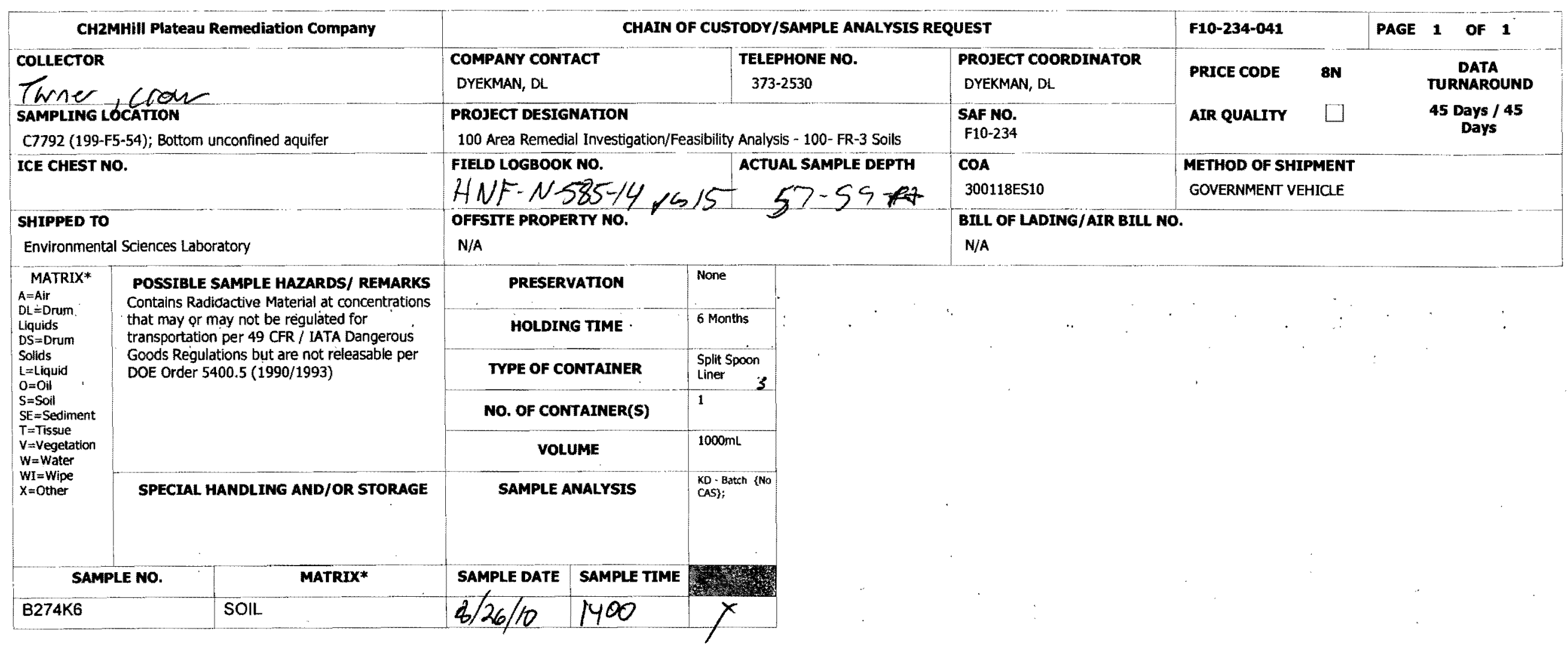

\section{CHAIN OF POSSESSION}

RELINQUISYED BY/REMOVED FRYM REA. Twent

SSU-R1

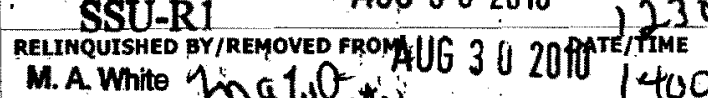

RELINQUISHED BY/REMOVED Frö

1400

RELINQUISHED BY/REMOVED FROM

DATE/TIME

DATE/TMME

RELINQUISHED BY/REMOVED FROM

RELINQUISHED BY/REMOVED FROM

DATE/TIME

DATE/TIME

\section{SIGN/ PRINT NAMES}

RECEIVED BY/STORED IN DATE/TIME RECEIVED BY/STORED IN $8 / 26 / 10$ 1525

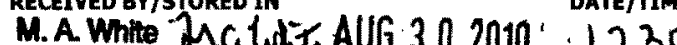
RECEIYER GX/STOREPIN

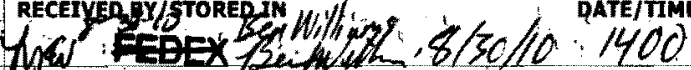
RECEIVED BY/STOREO IN RECEIVED BY/STORED IN DATE/TIME

RECEIVED BY/STORED IN

DATE/TIME

RECEIVED BY/STORED IN DATE/TIME
SPECIAL INSTRUCTIONS

** The CACN for all analytical work at WSCF laboratory is

401922ES20. $\square^{* *}$ The 100 Area S\&GRP Characterization and Monitoring

Sampling and Analysis GKI applies to this SAF.

\section{BRMA 13564}

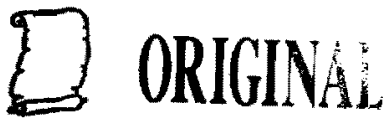

\begin{tabular}{c|c|}
\hline $\begin{array}{c}\text { LABORATORY } \\
\text { SECTION }\end{array}$ & RECEIVED BY \\
\hline $\begin{array}{c}\text { FINAL SAMPLE } \\
\text { DISPOSITION }\end{array}$ & DISPOSAL METHOD \\
\hline
\end{tabular}




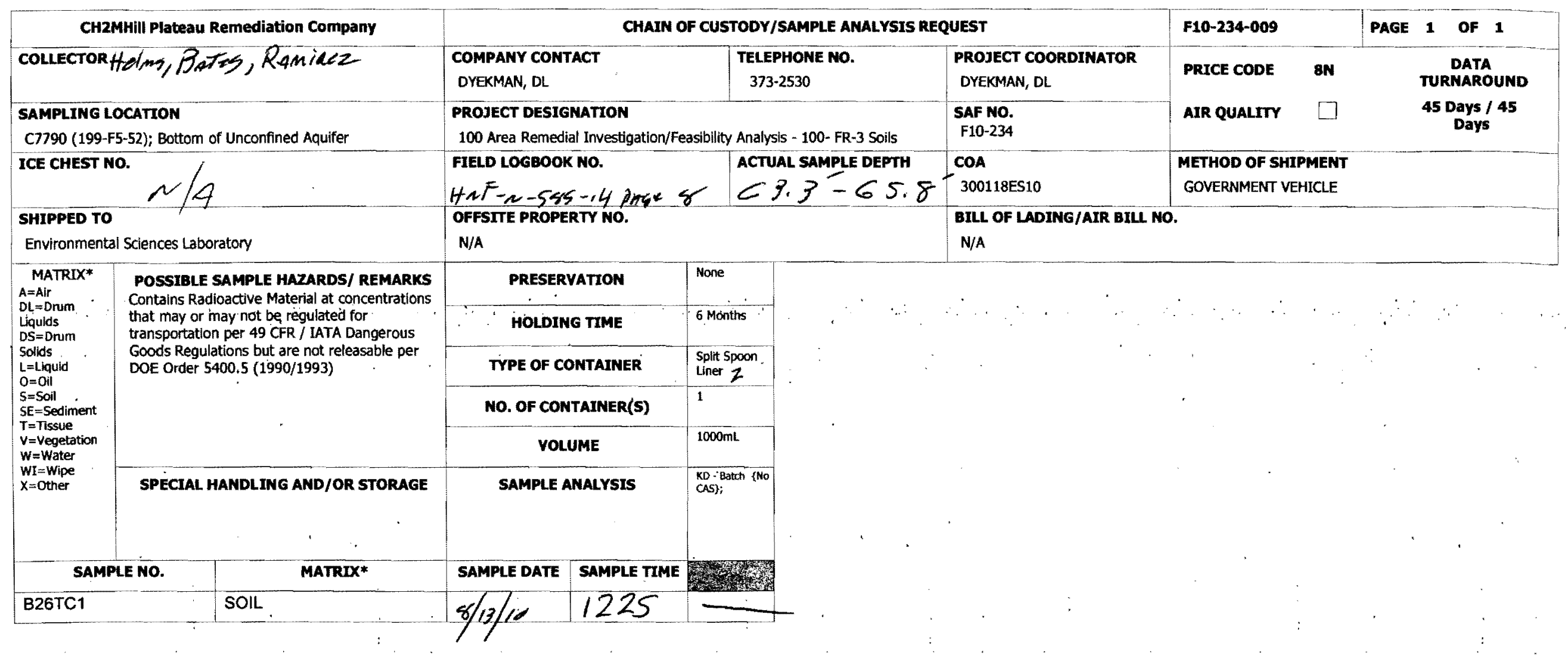

\begin{tabular}{|c|c|c|c|}
\hline \multicolumn{2}{|c|}{ CHAIN OF POSSESSION } & SIGN/ PRINT NAMES & \multirow{6}{*}{$\begin{array}{l}\text { SPECIAL INSTRUCTIONS } \\
\text { ** The } 100 \text { Area S\&GRP Characterization and Monitoring Sampling and } \\
\text { Analysis GKI applies to this SAF. } \\
\qquad \text { BRM\#/3564 }\end{array}$} \\
\hline \multirow{2}{*}{\multicolumn{2}{|c|}{ 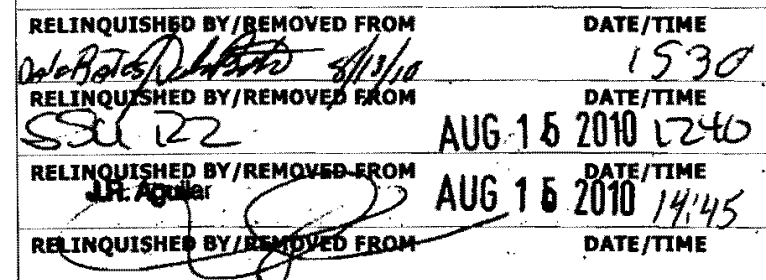 }} & 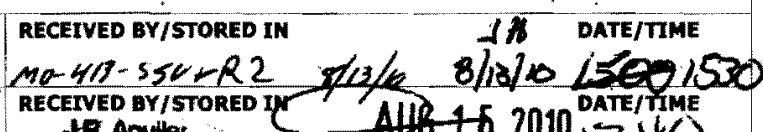 & \\
\hline & & 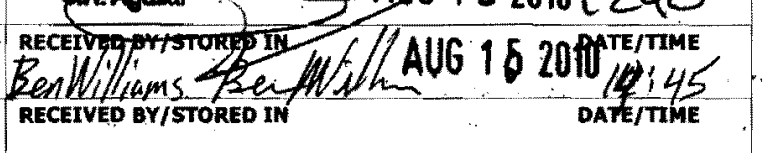 & \\
\hline \multicolumn{2}{|c|}{ RELIMQUTSHED BY/REMOVED FROM } & RECEIVED BY/STORED IN & \\
\hline \multicolumn{2}{|c|}{\begin{tabular}{|l} 
RELINQUISHED BY/REMOVED FROM \\
\end{tabular}} & RECEIVED BY/STORED IN & \\
\hline \multicolumn{2}{|c|}{ RELINQUISHED BY/REMOVED FROM } & RECEIVED BY/STORED IM & \\
\hline $\begin{array}{l}\text { LABORATORY } \\
\text { SECTTON }\end{array}$ & RECEIVED BY & & DATE/TIME \\
\hline $\begin{array}{l}\text { FINAL SAMPLE } \\
\text { DISPOSIIION }\end{array}$ & DISPOSAL METHOD & & DISPOSED BY \\
\hline
\end{tabular}




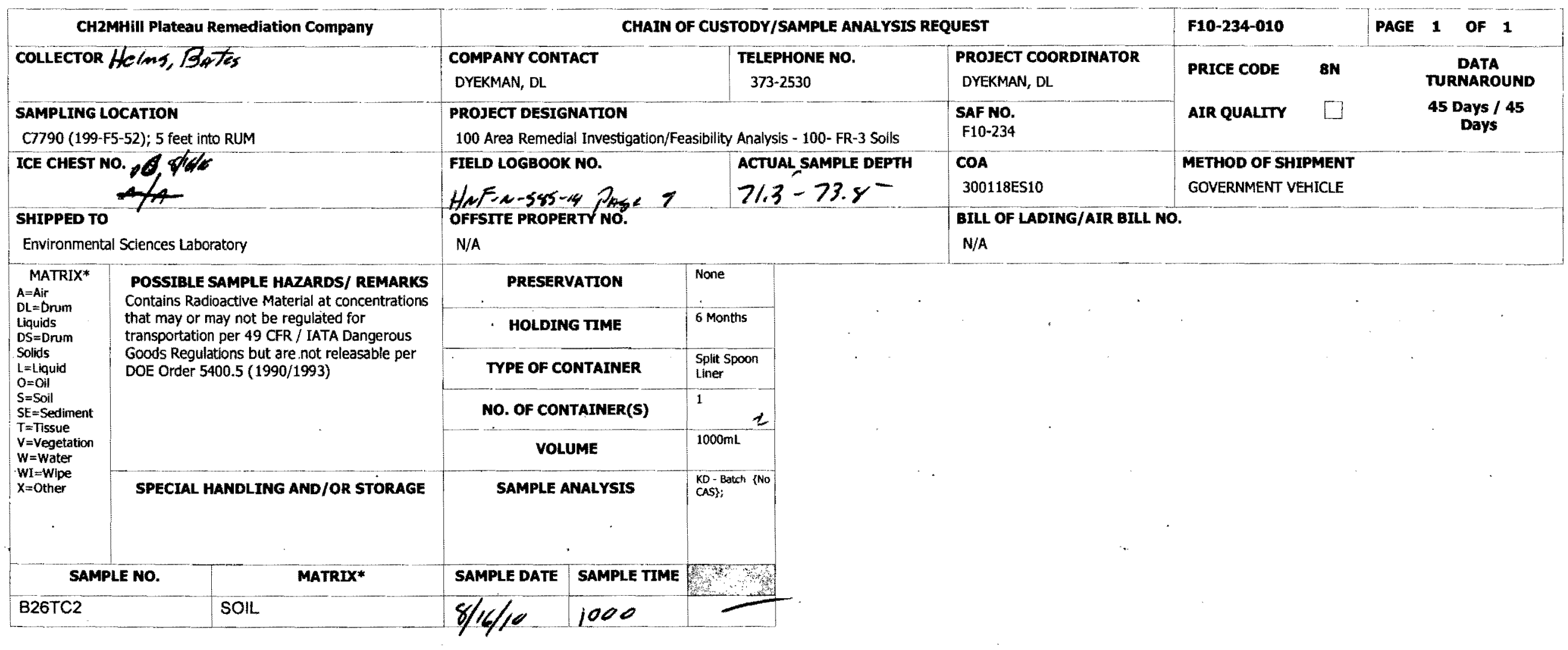

\begin{tabular}{|c|c|c|c|}
\hline \multicolumn{2}{|c|}{ CHAIN OF POSSESSION } & SIGN/ PRINT NAMES & \multirow{7}{*}{$\begin{array}{l}\text { SPECIAL INSTRUCTIONS } \\
\text { ** The } 100 \text { Area S\&GRP Characterization and Monitoring Sampling and } \\
\text { Analysis GKI applies to this SAF. } \\
\qquad R T M 13564\end{array}$} \\
\hline \multirow{3}{*}{\multicolumn{2}{|c|}{ 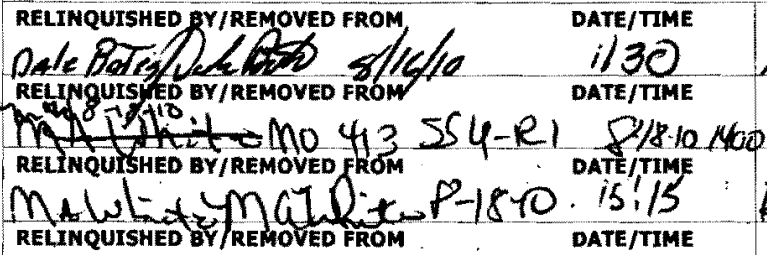 }} & 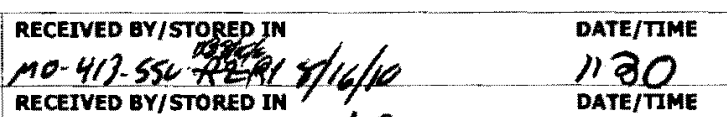 & \\
\hline & & MA whit methio 815101400 & \\
\hline & & 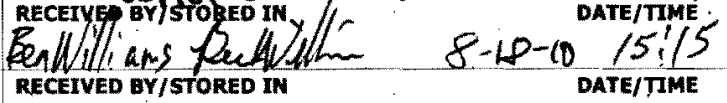 & \\
\hline \multicolumn{2}{|c|}{ RELINQUISHED BY/REMOVED FROM } & RECEIVED BY/STORED IN & \\
\hline \multicolumn{2}{|c|}{ RELIMQUISHED BY/REMOVED FROM } & RECEIVED BY/STORED IN & \\
\hline \multicolumn{2}{|c|}{ RELINQUISHED BY/REMOVED FROM } & RECEIVED BY/STORED IN & \\
\hline $\begin{array}{l}\text { LABORATORY } \\
\text { SECTION }\end{array}$ & RECEIVED BY & & DATE/TIME \\
\hline $\begin{array}{l}\text { FINAL SAMPLE } \\
\text { DISPOSITION }\end{array}$ & DISPOSAL METHOD & & DISPOSED BY \\
\hline
\end{tabular}

